\title{
FISH REMAINS FROM ARCHAEOLOGICAL SITES AS INDICATORS OF FORMER TRADE CONNECTIONS IN THE EASTERN MEDITERRANEAN
}

\author{
W. VAN NEER, O. LERNAU, R. FRIEDMAN, G. MUMFORD, J. POBLOME and M. WAELKENS
}

\begin{abstract}
The archaeozoological evidence that is available for the trade offish in the Eastern Mediterranean area is summarized. This overview covers the Chalcolithic to the Crusader period and is based on data from 76 sites compiled from the literature and from as yet unpublished material under study by the authors. Remains of fish from the Nile, the Mediterranean and the Red Sea are regularly found in archaeological sites that are located outside the natural distribution of the fish species and thus indicate that they were transported as items of trade or exchange. Evidence for trade of Anatolian freshwater fish is also documented. The fish bone finds are discussed according to their provenance and the regions to which they were transported, and an attempt is made, on the basis of archaeological, artefactual and - if available - texthal evidence, to understand the diachronic changes in the distribution within the broader economic and political contexts of the areas involved in the production and the consumption offish.
\end{abstract}

Résumé : Une synthèse des données archéozoologiques sur le commerce de poissons dans la partie orientale de la Méditerranée, depuis le Chalcolihique jusqu'au temps des croisades, est présentée. Cette revue est basée sur des données bibliographiques et sir des ensembles faniques inédits, étudiés par les auteurs, provenant de 76 sites. Des restes de poissons provenant du Nil, de la Méditerranée et de la mer Rouge sont fréquemment rencontrés sur des sites archéologiques situés en dehors de la distribution naturelle des espèces et indiquent dès lors qu'il s'agit de demées qui faisaient l'objet de commerce ou d'échange. Des indications sur le commerce de poissons d'eau douce d'Anatolie sont également présentées. Les trouvailles ichtyofauniques sont discutées en fonction de la provenance des poissons et des régions vers lesquelles ils étaient exportés. En utilisant les données archéologiques, les indications des artefacts et les informations historiques disponibles, les changements diachroniques observés dans le commerce de poisson sont décrits, en tenant compte di contexte économique et politique de chacune des régions productrice et consommatrice.

Key-Words : Archaeozoology, Fish, Trade, Eastem Mediterranean, Ichthyology.

Mots Clefs : Archéozoologie, Poissons, Commerce, Méditerranée orientale, Ichtyologie.

\section{ANIMAL REMAINS AS INDICATORS OF TRADE CONNECTIONS}

Former trade connections or other contacts among distant areas can be illustrated by means of animal remains belonging to species that did not occur in the vicinity of an archaeological site. Such allochthonous species can be imported at a site as living animals, as curiosities, as status symbols or as tributes, as fresh or preserved food items, as raw materials for the production of objects, as finished objects, or as items with a symbolic, religious or pecuniary value. Raw materials, such 
as antler or ivory, or objects made from skeletal parts, can arrive at a site as a result of complex and indirect trade or exchange processes and the same caveats usually apply to molluses'. In certain cases, it is clear that molluscs were imported as food items to a site, but usually the distance between the place of origin (mostly a coastal area) and the place of consumption (an inland site) is rather limited. An example of this in the region under consideration is the early Roman site of Mons Claudianus (Eastern Desert of Egypt) where more than 20 species of Red Sea species were found ${ }^{2}$, many of which can be considered as food items that were transported fresh over a distance of about $50 \mathrm{~km}$. In exceptional cases, however, molluscs can yield evidence for direct long distance trade. This is the case at Mons Claudianus and at Berenike, a Ptolemaic-Roman port site on the Red Sea coast, where remains of the edible snail (Helix pomatia) were found. The presence of opercula indicates that whole specimens arrived at these sites after a journey that took them from Italy to Alexandria, and from there to Middle Egypt along the Nile, from whence the snails were brought to the sites along the Eastern Desert roads ${ }^{3}$. Because of their aestivating period, which takes several months, these terrestrial snails are ideal for long distance transport and can, in addition, be delivered in a fresh state.

In the case of fish, the sites to which fresh specimens can be exported will depend on their distance from the place where the fishes are landed, on the means of transport available and on the temperature. In cold or temperate regions, commercializing fresh fish will be easier to accomplish than in warmer areas, and in both situations the winter season will be more suitable. When larger distances need to be crossed, the maximum travel distances are dictated by the efficiency of the preservation methods that prevent spoilage. Dehydration of fish (and meat) allows their long-term storage and therefore also their transport to distant areas. Preservation of fish by sun-drying, smoking and - in later periods - salting, probably has a long history. There is evidence available from Egypt that fish smoking already occurred long before the pharaonic period, when pictorial evidence documents this practice ${ }^{4}$. Late Palaeolithic Makhadma (Middle Egypt), dated to about $12000 \mathrm{BP}$, is a fish-smoking site where mainly tilapia was processed ${ }^{5}$. Fish drying stops bacterial decay and also pre-

\footnotetext{
1. See for instance REESE et al, 1986; REESE, 1991.

2. HAMLLTON-DYER, 2001.

3. VAN NEER and ERVYNCK, $1999: 339$.

4. E.g., VAN ELSBERGEN, 1997.

5. VAN NEER el al., 2000a,
}

vents infestation by flies, but the dried product may be attacked by other insects, mainly dermestid beetles. Still today, these animals are responsible for great losses of dried fish in subtropical areas ${ }^{6}$. However, once dried, fish can be stored for several months and thus may also be transported over fong distances. Salting or preparation of fish products in brine (salsumenta or fish sauces such as allec) allows even longer storage. Because of the seasonal abundance of fish, e.g., during the spawning season when the animals tend to aggregate in shallow waters, they can be easily captured in large numbers. This creates a surplus that can be prepared for future consumption or for export.

\section{FISH REMAINS AS INDICATORS OF TRADE : POSSIBILITIES AND CONSTRAINTS}

Fish bones found on an archaeological site are considered derived from imported fish when they belong to a taxon that did not occur in the vicinity of the site. It is therefore vital that the natural distribution of the fish species is well known. It is usually accepted that the present distribution roughly corresponds to the former one, but for certain periods and regions, it is obvious that this actualistic principle cannot be used. Climatic changes can alter the distribution as shown, for instance, at the Cave of Nerja near Malaga (Spain) where the presence of haddock (Melanogrammus aeglefinus) and pollack (Pollachins pollachitus) in layers dating to the Pleistocene-Holocene transitional period are a result of the lower palacotemperatures ${ }^{7}$. Today, haddock is a species characteristic of the North Atlantic that is rarely found south of the English Channel, and pollack only rarely enters the Mediterranean.

Diachronic shifting of coastlines also has to be taken into consideration when dealing with issues of fish trade. As a result of lowering sea levels or immense fluvial deposition causing the silting up of bays, the distance of present-day inland sites to the sea coast may have undergone changes over time. Such processes are well documented for the Persian Gulf ${ }^{8}$, but have not always been taken into account when interpreting finds of marine fish in Mesopotamian sites. The tuna finds from a Neo-Babylonian context at $\mathrm{Ur}^{9}$ are claimed

6. BLACHE and MITON, 1962.

7. RODRIGO GARCIA, 1994

8. RZÓSKA, 1980: 55 .

9. ELLISON et al., 1978. 
to illustrate transport over a distance of at least $180 \mathrm{~km}$. However, during the $1^{\text {st }}$ millennium $\mathrm{BC}$, Ur was located only about $80 \mathrm{~km}$ from the coastline. Similarly, the former position of the coastline ${ }^{10}$ has to be taken into account when evaluating the older Early Dynastic and Akkadian finds of marine fish from Abu Salabikh ${ }^{11}$, Isin-Isan Bahriyat ${ }^{12}$, Lagash ${ }^{13}$, and UrukWarka ${ }^{14}$. During the $3^{\text {rd }}$ nillennium BC, Lagash was located at the seashore and Uruk-Warka was close to the coast. The other sites were located inland, but at a relatively short distance (maximum 80-100 km) from the coast in the past. Nowadays, the seashore is about $250 \mathrm{~km}$ from these sites. The distance to the coast will certainly have consequences for the interpretation of the way the fish were transported (fresh or preserved). Moreover, it should be stressed that certain fish taxa that were identified from these inland sites have a relatively high tolerance of salinity variation. As a result of this, fish, such as the Mugilidae (mullets) from Abu Salabikh and Isin-Isan Bahriyat or the Hilsa ilisha from Abu Salabikh, may well represent local catches rather than imported food items. A good knowledge of the behaviour and ecological tolerance of fish species is therefore also vital for correct interpretation.

In some instances information on the modern biology is insufficient for accurate interpretation. This is the case for the sawfish (Pristis sp.), for which finds exist at Hassek Höyïk in south-eastern Anatolia ${ }^{15}$. At this site located along the Euphrates, sawfish remains have been found in Chalcolithic and Early Bronze Age levels. Sawfish are known to enter rivers, but because of their vulnerability to over-fishing, numbers have declined dramatically in recent times, and it remains unclear how far they may have been able to ascend the Euphrates in the past. Hassek Höyuk is at a distance of about $1400 \mathrm{~km}$ from the Gulf, which seems too far to suggest the possibility that the sawfish were captured locally. However, more or less contemporaneous finds of vertebrae and a rostrum have been found at Susa ${ }^{16}$; moreover, modern specimens of a South American species (Pristis perotteti) have been found occasionally in the Amazon river about $1300 \mathrm{~km}$ from the coast ${ }^{17}$. Similarly, it is unclear what the exact former distribution of the sturgeon (Acipenser sturio) was in the

10. Cf. maps in RZÓSKA, $1980: 55$.

11. VON DEN DRIESCH, $1986 \mathrm{a}$.

12. BOESSNECK, 1977 ; VON DEN DRIESCH, 1981 ; BOESSNECK and ZIEGLER, 1987.

13. MUDAR, 1982.

14. BOESSNECK et al., 1984.

15. STAHL, 1989 ; BOESSNECK, 1992.

16. BOESSNECK, 1992.

17. BIGELOW and SCHROEDER, $1953: 41$; THORSON, 1974.
Mediterranean, making it difficult to establish the origin of the Roman-early Byzantine finds of Acipenseridae at Sagalassos, Turkey ${ }^{18}$. Members of the sturgeon family occur in the Black Sea, the Sea of Marmara and the adjacent rivers, such as the Sakarya, the Kızil Irmak and the Yeşil Irmak. Of the four species known to the region, only the common sturgeon has a more southerly distribution; it occurs also in the Aegean Sea. Today, Acipenser sturio does not live along the southern coasts of Turkey, but historical evidence seems to indicate that the species was found here in antiquity ${ }^{19}$. It is unclear whether natural or anthropogenic factors should be held responsible for this local extinction.

Recent human influences on the distribution patterns exist and can seriously hamper interpretation. Since the identification of the place of origin of traded fish depends on a good knowledge of the original distribution of the species, it may in certain cases be necessary to carry out additional fieldwork. This is needed for regions in which the ichthyofaunas have been insufficiently explored. In the case of Sagalassos, for instance, an exploration of the freshwater fish fauna of the site's territory and the regions beyond it had to be carried out in order to fill the gaps in the existing literature ${ }^{20}$. There were difficulties in establishing the original distribution for carp (Cyprinus carpio), which was the most common food fish at Sagalassos. During the last few decades, the artificially introduced domestic form has been interbreeding with the wild form ; moreover, dams were built in certain areas and the slow running or stagnant waters were stocked with carp. Other difficulties encountered during the reconstruction of the original distribution patterns included the changing water regimes of rivers due to excessive pumping for irrigation and the devastating effect of the introduction of pikeperch (Sander lucioperca) that is responsible for massive decline and sometimes even local extinction of species.

Another species whose former natural distribution has sometimes been considered problematic is the Nile perch (Lates niloticus). It is usually considered a typical African species, but as a result of the numerous finds in sites in Israel, it was suggested by $\mathrm{H}$. Lernau ${ }^{21}$ that it may have had a wider distribution in the past and may have occurred in the coastal rivers of Israel. Zoogeographical arguments for this hypothesis include the presence in the Levant of other African aquatic species such as the Nile catfish Clarias gariepinus, a tilapia

18. VAN NEER el al., 1997.

19. NOLLÉ, 1993.

20. VAN NEER et al., 2000b; VAN NEER $e t$ al., in press.

21. LERNAU, 1986-1987. 
species (Sarotherodon galilaets), and the soft-shelled turtle Trionyx trinnguis. The hippopotamus Hippopotamus amphibius and the crocodile Crocodylus miloticus are two other such species, but they disappeared in historic times. An alternative explanation for the presence of Nile perch at sites in Israel is that they were imported as food from the Nile. It is indeed striking that most finds of Lates niloticus date to the Bronze Age and younger periods, when commercial relationships with Egypt were well established. Except for a single Chalcolithic find from Tel Katif22, there are no older archaeozoological records for the species in Israel. In a palaeontological context, the genus Lates has been recorded in the Levant only from early Miocene deposits in the northern Negev23. As it now stands, trade seems to be the most likely explanation since Lates niloticus is being found on more and more archaeological sites in the Eastern Mediterranean, e.g., in Turkey and Cyprus which are outside the region where the other aforementioned African elements occur. In addition, Nile perch is often found in association with other Nilotic taxa, such as Synodontis, Bagrus, and sometimes Mormyridae and Distichodus. In order to settle this question in a more definite way, it would be useful to try geochemical, isotopic or genetic analyses. Such analyses also have potential in cases when traded fish belong to a taxon that has a wide distribution.

In certain cases, the identification of fish remains cannot go beyond the level of family, either because the represented skeletal elements are not sufficiently diagnostic or because the researcher did not have a complete reference collection of that particular group for accurate identification. Finds identified as belonging to the family Serranidae or even the genus Epinephelus have little value for establishing origin since these fish occur in the Mediterranean, the Red Sea and the Gulf. However, in certain cases, circumstantial evidence can help to suggest a likely place of origin for fish with a wide distribution. At Sagalassos, for instance, seven marine taxa were identified, of which four live both in the Mediterranean and the Black Sea. The remaining three taxa occur in the Mediterranean but not in the Black Sea. Because there is only positive evidence for Mediterranean imports, it is assumed that all marine fish discovered at this site came from the Mediterranean coast which is, in addition, much closer than the Black $\mathrm{Sea}^{24}$. Moreover, Sagalassos was also located on the road on which the Docimian marble and sarcophagi were taken to the

22. LERNAU, 1996.

23. GOLDSMITH et al., 1982.

24. VAN NEER et al., 1997.
Pamphylian coast ${ }^{25}$, and coin finds at Sagalassos suggest that especially the port of Perge was used for export and import by the Sagalassians ${ }^{26}$. The argument of closest proximity is often used to attribute an origin to imported fish. At Petra, for instance, it is supposed that all the marine fish came from the Red Sea ${ }^{27}$. There are indeed a few taxa that are typical for that region, but there are also fish that are found in both the Mediterranean and the Red Sea.

Among the freshwater fish, species also occur that have a wide distribution, and this hampers a determination of their origin. In one instance, it has been possible to define the geographic origin of imported fish by genetic analysis. This is the case for the Clarias catfish finds from the Roman-early Byzantine site of Sagalassos. On zoogeographical grounds, these fish could have been imported from either the Nile or from the Levant, and circumstantial evidence of an archaeological and epigraphical nature was equivocal. Ceramic and numismatic evidence show that contacts with both regions existed, and the epigraphic data indicate that the aristocracy of Sagalassos served in the Roman army and the provincial administration of Syria, Palestina and Egypt during the imperial period. Mitochondrial DNA analysis was carried out on modern populations of Clarias gariepinus from Turkey, Syria, Israel and Egypt. Ancient DNA could be successfully extracted, amplified and sequenced from the archaeological specimens. Comparison of the ancient and modern haplotypes indicates that the ancient catfish bones originated from the lower Nile ${ }^{28}$. Stable isotope analysis is another method that may help to elucidate the geographic origin of fish species with a wide geographic distribution. At Sagalassos, wild carp (Cyprimus carpio) is the most common freshwater species consumed at the site $^{29}$. The reconstruction of the original distribution of the wild carp is difficult because of the systematic stocking of carp in Turkish waters that are suitable for this species. On the basis of a literature survey and fieldwork, it is believed that the nearest water body at which carp was available in antiquity was Lake Eğirdir. However, the species was probably also present in Lakes Beyşehir, Akşehir and Eber. The study of oxygen isotopes may indicate the precise origin of the fish consumed at Sagalassos, since the lakes where the species lived are situated at different altitudes. Preliminary analyses of water samples already show different oxygen isotope

25. WAELKENS, 1982 ; WAELKENS et al., 2002.

26. WVAELKENS, 2002.

27. DESSE-BERSET and STUDER, 1996.

28. ARNDT et al., 2003.

29. VAN NEER et al., 1997. 
ratios, and in a next step, modern and archaeozoological specimens will be analysed. Other isotopes, e.g., strontium, may also have potential to aid in the identification of the place of origin of fish ${ }^{30}$. Attempts are being made to use this kind of analyses to settle the issue of the putative extended distribution of Nile perch in the Levant. ${ }^{31}$

Certain fish have a limited distribution and this makes them ideal indicators of former trade connections. Such species are, for example, the endemic fish found in Anatolian lakes, which developed as a result of long geographical isolation. In the Roman-early Byzantine site of Sagalassos, remains were found of Psendophoxinus handlirschil, a species that only occurs in Lake Egirdir today ${ }^{32}$. Finding such unequivocal indications of a very precise origin as in this case relies mainly on recovering bones that are sufficiently diagnostic for identification at species level. In the case of the numerous Anatolian cyprinid finds from Sagalassos, wellpreserved pharyngeal tooth plates or basioccipitats are the most suitable elements for precise identification.

Another point that needs to be mentioned is the problem that can arise when using identifications of fish taxa mentioned in some of the literature. Sometimes it is obvious that an identification cannot be as precise as indicated on the basis of osteological criteria alone. Clarias gariepinus finds reported from sites in the Levant and Egypt, for instance, can be accepted as belonging osteomorphologically to the family Clariidae. A gemus identification (Clarias sp. or Heterobranchus sp.) is, however, only possible from a few skeletal elements ${ }^{33}$, of which the pectoral spine is most frequently used. To arrive at a species level identification within the genus Clarias, only the vomer tooth plate allows a distinction to be made between Clarias gariepinus and Clarias anguillaris ${ }^{34}$, and for the two species within the genus Heterobranchus no diagnostic criteria have been described. In the Egyptian Nile, Clarias is indeed the most common genus in archaeological contexts, and the few specific identifications of such material are all Clarias gariepinus. Today this species is still the most common clariid in the Egyptian Nile and it is the only species living in the Levant. Thus the attribution of archaeological specimens to Clarias gariepimus will in most cases be correct. However, the identification of clariids from the Levant as Heterobranchus or Clarias anguillaris has been excluded

\footnotetext{
30. VAN NEER et al., 2004 ; DUFOUR el al., in prep.

31. REINHARDT et al, in prep.

32. VAN NEER $e t$ al., 2000b ; VAN NEER $e l$ al., in press.

33. VON DEN DRIESCH, 1983.

34. GAUTIER and VAN NEER, 1989.
}

a priori since those taxa - which would be an indication of trade with Egypt - have never been taken into consideration. Similarly, finds of Trachurus trachurus have been described from Upper Zohar ${ }^{35}$ in Israel and were considered imports from the Mediterranean, but it appears that Trachurus indicus, the species living in the Red Sea, had not been taken into account at the time of analysis ${ }^{36}$. The Epinephelus aeneus from En Boqeq and Tamara ${ }^{37}$ were identifications based on an apparent perfect match with the modern specimen in the comparative collection, but the Epinephelus species from the Red Sea were not available for comparison at that time. On the other hand, it must be stated that the finds of Red Sea parrotfish (Scaridae) mentioned from many sites in the Levant are definitely not Mediterranean, since they differ morphologically from Sparisoma cretensis, the only scarid species living in the Mediterranean ${ }^{38}$.

In some cases, finally, details for determining the place of origin of the imported fish are lost due to the presentation of the data in publications. The fish remains from Tell Hesban date between the Iron Age II and the modern period and were described in great detail by Lepiksaar ${ }^{39}$, but the archaeological find spots refer to excavation squares and loci which cannot be linked to a chronological period based on the publication. A discussion of the diachronic changes in the fish fauna is provided in another chapter by von den Driesch and Boessneck ${ }^{40}$, but this summary only mentions the finds at family level. As a result of this, it is not possible to collect information that might help to distinguish the precise geographic origin of certain fishes, i.e., the Scombridae species. Nevertheless, it is clear that in all periods fish came from three different sources : freshwater - probably the Jordan basin, the Mediterranean and the Red Sea.

\section{THE DATABASE OF TRADED FISH}

The discussion of the various traded fish in the different regions presented below is based on data collected from the literature and on unpublished data of our own or from colleagues. Tables 1-6 provide for each site the imported taxa,
35. LERNAU, 1995.
36. O. Lemau, pers. obs.
37. LERNAU, 1986.
38. E.g., LERNAU, 1986.
39. LEPIKSAAR, 1995.
40. VON DEN DRIESCH and BOESSNECK, 1995. 
their place of origin as mentioned in the publication and their chronological context. The distance of the sites to the possible source areas has been added. In the case of Red Sea and Mediterranean fish, the shortest direct distance to the coast was calculated. For the Nilotic fish, it was not always clear whether the fish were transported by sea or over land. Nevertheless, to give a general idea, we measured the distance between the find spot and a mid-point in the Egyptian Delta, namely the town of Zagazig. In the case of transport into the Eastern or Western Desert of Egypt, the shortest distance to the Nile was taken. A column is also added in tables 3-6 indicating our alternative interpretation of the place of origin. The bibliographic references are indicated in the tables, and will not be repeated during the discussion of the trade patterns.

Recently, it has been argued ${ }^{41}$ that transport of fish in a fresh state can be presumed for late medieval sites in Europe that are located $150 \mathrm{~km}$ or less from the coast, which is the distance that can be adequately covered by swift transport using horses. Among the sites listed in the tables, there are a few coastal settlements and sites that are only a relatively short distance from the Mediterranean coast. In those cases, it was judged umnecessary to list all the marine species that were found at the site, and instead simply the number of Mediterranean taxa is indicated. Although we are aware that terrain can affect the speed of transport, we assume that fish could be traded in a fresh state over a distance of about $50 \mathrm{~km}$, this being a generally accepted distance that could be covered in a day by two individual parties meeting at a central point. This distance serves to differentiate regional from wider distribution patterns ${ }^{42}$. When distances were larger, it is likely that the fish were transported in a preserved form.

\section{TRADE IN NILOTIC FISH}

Nilotic fish taxa that have thus far been mentioned as exports from the Nile Valley are Nile perch (Lates niloticus) and Clariidae catfish (mainly designated as Clarias sp. or Clarias gariepinus in the literature), whereas finds of Synodontis catfish, Bagrus catfish, tilapia, and especially barbels (Barbus sp. and Labeo sp.), mormyrids (Mormyridae) and Distichodus have been less frequently reported in the literanure. The fish remains compiled in the present contribution

41. HOFFMANN, $2001: 141$.

42. HODDER and ORTON, $1976: 20-29$. yield evidence for transport within the boundaries of Egypt and for trade from Egypt with the Syro-Palestinian area, some of the eastern Mediterranean islands and Anatolia (fig. 1).

\section{INSIDE EGYPT}

Evidence to date for the export of Nile fish to sites in the Western Desert is limited to the Clarias finds from Dakhleh oasis, which are dated to the Old Kingdom (table 1). The presence of Nile perch has been reported from a Nabta/Al Jerar Neolithic context ( $c a 8000-7300$ BP) at Nabta Playa, located at a distance of almost $200 \mathrm{~km}$ from the Nile. These bones were initially considered an indication of the import of dried Nilotic fish ${ }^{43}$, but despite the presence of imported Nile molluses, Gautier ${ }^{44}$ believes instead they represent reworked intrusives from older lake deposits, such as the ones at Bir Tarfawi dating to the last interglacial period ${ }^{45}$. Conditions were more humid during that interglacial period than in the early Holocene. The presence of Nile perch and other freshwater fish in the Western Desert during the last interglacial period was a result of hydrographical connections that existed with the Nile Valley ${ }^{46}$. The finds of Clarias reported by Wheele 47 from a first century AD context at Sidi Krhebish, Benghazi in Libya were considered imports from the Nile ${ }^{48}$; however, it appears that this catfish also lives in Lake El Hombra located $10-15 \mathrm{~km}$ northeast of Benghazi $\mathrm{i}^{49}$.

Transport of preserved Nilotic fish into the Eastern Desert is documented by finds dating to the late Ptolemaic and Roman periods. At Kasarvit, several Nilotic fish were found that most probably came from the Pelusiac branch of the Nile ${ }^{50}$, which is located about $25 \mathrm{~km}$ west of the site (table 2). Finds dating to both the Ptolemaic and the late Roman period have been discovered here. Mons Claudianus, a first-second century AD quarry site, has yielded a concentration of small specimens of cyprinids (Labeo sp.), catfish (Clarias, Synodontis, and Bagrus) and mormyrids that have been interpreted as the remnants of pickled fish. Mons Porphyrites, another early Roman quarry site, yielded a few bones of Nile perch

\footnotetext{
43. VAN NEER, 1989.

44. GAUTIER, 2001.

45. VAN NEER, 1993.

46. WENDORF et al., 1993.

47. WHEELER, 1979.

48. See also REESE et al., 1986

49. VINCIGUERRA, 1922.

50. SNEH and WEISSBROD, 1973.
} 


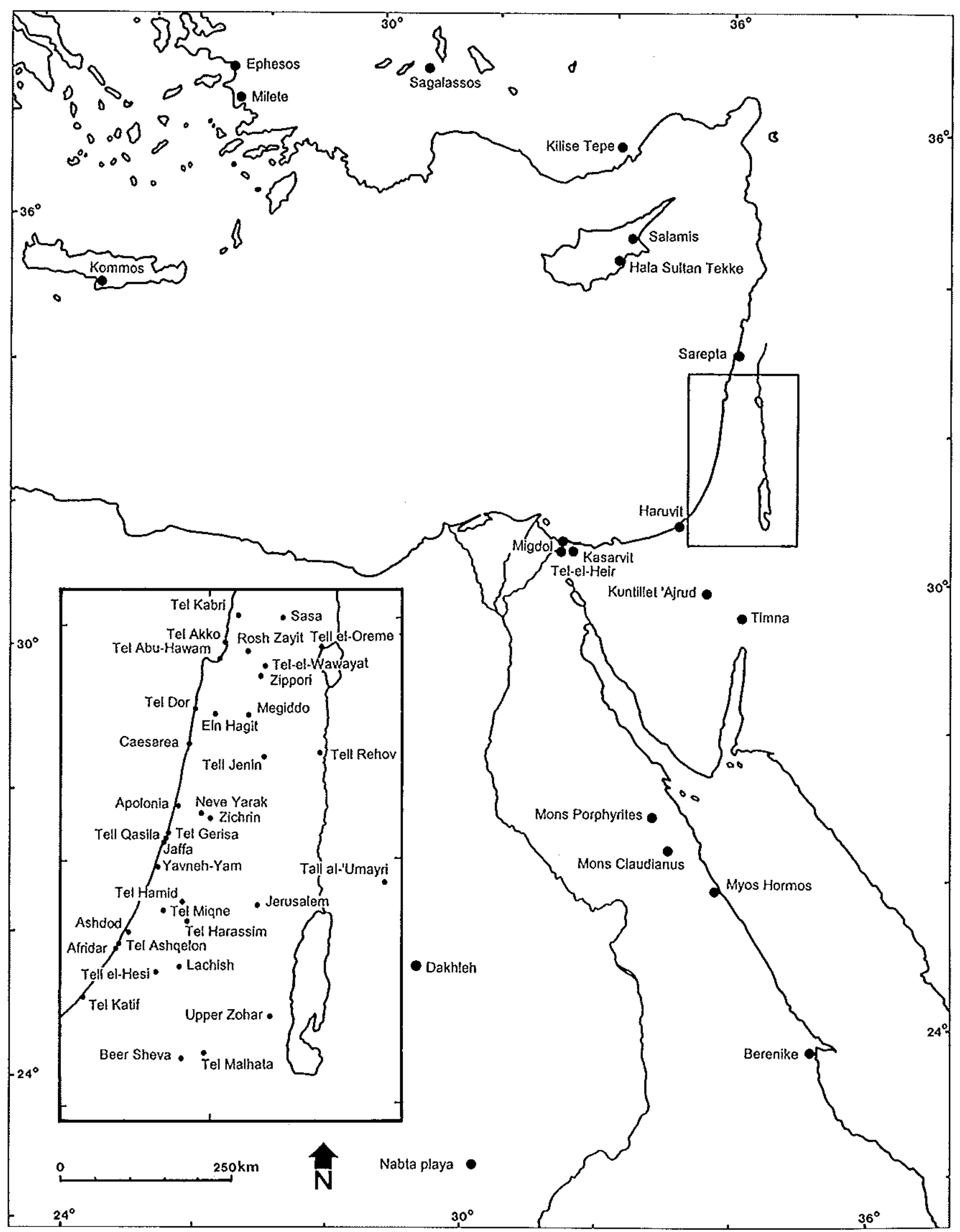

Fig. 1 : Sites in the Eastern Mediterranean with indication for import of Nilotic fish. 
Table 1: Evidence for fish trade in sites from the Egyptian Eastern and Western Desert.

\begin{tabular}{|c|c|c|c|}
\hline SITE & PERIOD & TAXON & ORIGIN IN PUBLICATION \\
\hline Nabta playa ${ }^{a}$ & Neolithic & Lates niloticus (Nile perch) & Nile $(200 \mathrm{~km})^{\mathrm{b}} / \mathrm{local}^{\mathrm{c}}$ \\
\hline Dakhleh oasis & Old Kingdom & Clarias sp. (catfish) & Nile $(290 \mathrm{~km})$ \\
\hline Berenike & Late Ptolemaic & Clarias sp. (catfish) & Nile $(300 \mathrm{~km})$ \\
\hline \multirow[t]{2}{*}{ Berenike $^{f}$} & Early Roman & $\begin{array}{l}\text { Clarias sp. (catfish) } \\
\text { Bagrus sp. (catfish) } \\
\text { Synodontis sp. (catfish) } \\
\text { Schilbe mystus (catfish) }\end{array}$ & $\begin{array}{l}\text { Nile }(300 \mathrm{~km}) \\
\text { Nile }(300 \mathrm{~km}) \\
\text { Nile }(300 \mathrm{~km}) \\
\text { Nile }(300 \mathrm{~km})\end{array}$ \\
\hline & Late Roman & $\begin{array}{l}\text { Bagrus sp. (catfish) } \\
\text { Synodontis sp. (catfish) }\end{array}$ & $\begin{array}{l}\text { Nile }(300 \mathrm{~km}) \\
\text { Nile }(300 \mathrm{~km})\end{array}$ \\
\hline Myos Hormos' & Early Roman & Barbus sp. (barbel) & Nile $(160 \mathrm{~km})$ \\
\hline Mons Claudianush & Early Roman & $\begin{array}{l}\text { Carcharhinidae (requiem sharks) } \\
\text { Serranidae (groupers) } \\
\text { Lutjanidae (snappers) } \\
\text { Sparidae (seabreams) } \\
\text { Lethrinidae (emperors) } \\
\text { Carangidae (trevallies and jacks) } \\
\text { Labridae (wrasses) } \\
\text { Scaridae (parrotfish) } \\
\text { Mugilidae (mullets) } \\
\text { Sphyraenidae (barracudas) } \\
\text { Acanthuridae (surgeonfish) } \\
\text { Balistidae (triggerfish) } \\
\text { Mormyridae (elephant-snout fish) } \\
\text { Clarias sp. (catfish) } \\
\text { Bagrus sp. (catfish) } \\
\text { Symodontis schall (catfish) } \\
\text { Labeo sp. (barbel) } \\
\text { Tilapiini (tilapia) }\end{array}$ & $\begin{array}{l}\text { Red Sea }(50 \mathrm{~km}) \\
\text { Red Sea }(50 \mathrm{~km}) \\
\text { Red Sea }(50 \mathrm{~km}) \\
\text { Red Sea }(50 \mathrm{~km}) \\
\text { Red Sea }(50 \mathrm{~km}) \\
\text { Red Sea }(50 \mathrm{~km}) \\
\text { Red Sea }(50 \mathrm{~km}) \\
\text { Red Sea }(50 \mathrm{~km}) \\
\text { Red Sea }(50 \mathrm{~km}) \\
\text { Red Sea }(50 \mathrm{~km}) \\
\text { Red Sea }(50 \mathrm{~km}) \\
\text { Red Sea }(50 \mathrm{~km}) \\
\text { Nile }(110 \mathrm{~km}) \\
\text { Nile }(110 \mathrm{~km}) \\
\text { Nile }(110 \mathrm{~km}) \\
\text { Nile }(110 \mathrm{~km}) \\
\text { Nile }(110 \mathrm{~km}) \\
\text { Nile }(110 \mathrm{~km})\end{array}$ \\
\hline Mons Porphyrites ${ }^{\mathrm{i}}$ & Early Roman & $\begin{array}{l}\text { Carcharhinidae (requiem sharks) } \\
\text { Muraenidae (moray eels) } \\
\text { Serranidae (groupers) } \\
\text { Carangidae (trevallies and jacks) } \\
\text { Lutjanidae (snappers) } \\
\text { Lethrinidae (emperors) } \\
\text { Sparidae (seabreams) } \\
\text { Labridae (wrasses) } \\
\text { Scaridae (parrotfish) } \\
\text { Acanthuridae (surgeonfish) } \\
\text { Balistidae (triggerfish) } \\
\text { Mugilidae (mullets) } \\
\text { Clarias gariepinus (catfish) } \\
\text { Bagrus docmak (catfish) } \\
\text { Synodontis schall (catfish) } \\
\text { Lates niloticus (Nile perch) }\end{array}$ & $\begin{array}{l}\text { Red Sea }(50 \mathrm{~km}) \\
\text { Red Sea }(50 \mathrm{~km}) \\
\text { Red Sea }(50 \mathrm{~km}) \\
\text { Red Sea }(50 \mathrm{~km}) \\
\text { Red Sea }(50 \mathrm{~km}) \\
\text { Red Sea }(50 \mathrm{~km}) \\
\text { Red Sea }(50 \mathrm{~km}) \\
\text { Red Sea }(50 \mathrm{~km}) \\
\text { Red Sea }(50 \mathrm{~km}) \\
\text { Red Sea }(50 \mathrm{~km}) \\
\text { Red Sea }(50 \mathrm{~km}) \\
\text { Red Sea }(50 \mathrm{~km}) \\
\text { Nile }(130 \mathrm{~km}) \\
\text { Nile }(130 \mathrm{~km}) \\
\text { Nile }(130 \mathrm{~km}) \\
\text { Nile }(130 \mathrm{~km})\end{array}$ \\
\hline El-Zarkehj & Early Roman & Scaridae (parrotfish) & Red Sea $(65 \mathrm{~km})$ \\
\hline \multicolumn{2}{|c|}{$\begin{array}{l}\text { a. GAUTIER, } 1980 \text {; VAN NEER, } 1989 \text {; GAUTIER, } 2001 . \\
\text { b. GAUTIER, } 1980 \text {; VAN NEER, } 1989 . \\
\text { c. GAUTIER, } 2001 \text {. } \\
\text { d. MLLL, } 2002 \text {. } \\
\text { e. VAN NEER, unpublished. }\end{array}$} & \multicolumn{2}{|c|}{$\begin{array}{l}\text { f. VAN NEER and ERVYNCK, 1998, } 1999 . \\
\text { g. VAN NEER, pers. observation. } \\
\text { h. HAMILTON-DYER, 1994, } 2001 . \\
\text { i. HAMLLTON-DYER, in press. } \\
\text { j. LEGULLOUX, } 1997 .\end{array}$} \\
\hline
\end{tabular}


Table 2 : Evidence for fish trade in sites from the Egyptian Nile valley and Delta area.

\begin{tabular}{|c|c|c|c|}
\hline SITE & PERIOD & TAXON & ORIGIN IN PUBLICATION \\
\hline \multirow[t]{3}{*}{ Tell el-Daba'a } & $\begin{array}{l}\text { Middle Kingdom - Second Intermediate Period } \\
(2055-1550 \mathrm{BC})\end{array}$ & Argyrosomus regitis (meagre) & Mediterranean $(40 \mathrm{~km})$ \\
\hline & & Sparus auratus (gitthead seabream) & Mediterranean $(40 \mathrm{~km})$ \\
\hline & & Dicentrarchus punctatus (sea bass) & Mediterranean $(40 \mathrm{~km})$ \\
\hline \multirow[t]{2}{*}{ Tel-el-Heirb } & Persian/Hellenistic & Sparidae (seabreams) & Mediterranean $(12 \mathrm{~km})$ \\
\hline & & Mugilidae (mullets) & Mediterranean (12 km) \\
\hline \multirow[t]{2}{*}{ Tel Maskhuta ${ }^{\mathrm{c}}$} & Late Period ( $7^{\text {th }} \mathrm{c} \mathrm{BC}$ ) to Roman period & Sparus aurata (gilthead seabream) & Mediterranean $(55 \mathrm{~km})$ \\
\hline & & Sciaenidae (drums) & Mediterranean $(55 \mathrm{~km})$ \\
\hline Migdold & Iron Age II $\left(6^{\text {th }} \mathrm{c} \mathrm{BC}\right)$ & Sparidae (seabreams) & Mediterranean $(15 \mathrm{~km})$ \\
\hline Kasarvite & Ptolemaic & $\begin{array}{l}\text { Clarias gariepinus (catfish) } \\
\text { Bagrus sp. (catfish) } \\
\text { Lates niloticts (Nile perch) } \\
\text { Cichlidae } \\
\text { Cyprinidae (barbels) } \\
\text { at least } 7 \text { marine taxa }\end{array}$ & $\begin{array}{l}\text { Pelusiac branch of Nile }(25 \mathrm{~km}) \\
\text { Pelusiac branch of Nile }(25 \mathrm{~km}) \\
\text { Pelusiac branch of Nile }(25 \mathrm{~km}) \\
\text { Pelusiac branch of Nile }(25 \mathrm{~km}) \\
\text { Pelusiac branch of Nile }(25 \mathrm{~km}) \\
\text { Mediterranean }(12 \mathrm{~km})\end{array}$ \\
\hline & Late Roman & $\begin{array}{l}\text { Lales niloticus (Nile perch) } \\
\text { Cyprinidae (barbels) } \\
\text { at least } 11 \text { marine taxa }\end{array}$ & $\begin{array}{l}\text { Pelusiac branch of Nile }(25 \mathrm{~km}) \\
\text { Pelusiac branch of Nile }(25 \mathrm{~km}) \\
\text { Mediterranean }(12 \mathrm{~km})\end{array}$ \\
\hline Shenhourf & Coptic & Scaridae (parrotfish) & Red Sea $(160 \mathrm{~km})$ \\
\hline
\end{tabular}
a. VON DEN DRIESCH, 1986b.
b. LERNAU, unpublished.
d. LERNAU, unpublished.
c. VON DEN DRIESCH, $1986 \mathrm{~b}$.
e. Ibid.
f. QUAEGEBEUR and TRAUNECKER, 1994 ; VAN NEER, in preporation.

and three catfish taxa (Clarias gariepinus, Synodontis schall and Bagrus docmak). These were brought from the Nile Valley along the desert roads by caravans servicing the imperial stone quarries with foodstuffs and other basic daily necessities $^{51}$. The same mechanism, be it as a by-product of the Red Sea trade, explains the presence of salsamenta of small Barbus sp. recently found at early Roman Myos Hormos, on the Red Sea coast. Besides these smaller fish, larger specimens were also transported along the Eastern Desert roads. Late Ptolemaic contexts at Berenike, a harbour site on the Red Sea coast, yielded remains of Clarias catfish. In early Roman levels of that site, four Nilotic catfish were found (Clarias, Synodontis, Schilbe and Bagrus), and late Roman contexts yielded Bagrus and Symodontis. The finds of Nilotic fish at sites along the Red Sea, where plenty of marine fish was available for consumption, is striking. It is unclear whether this reflects the desire to satisfy the culinary preferences of people who inhabited these remote desert stations, but originated from the Nile Valley, or if it was simply a result of commercial reasoning. No doubt the caravans had larger loads to transport from the Red Sea harbours to the Nile Valley than in

51. SIDEBOTHAM and ZiTTERKOPF, 1995. the other direction. Perhaps dried fish were simply one of the commonly available goods that were taken along the desert roads to fill the wagons, but were not major items in or the impetus for the trade caravans. The way in which regional produce interlocked with more wide ranging exchange patterns, such as the international Red Sea trade ${ }^{52}$, in which Rome's interest was limited to the tax revenue, or the annona provisioning the Roman military ${ }^{53}$ or the metropoleis of Alexandria ${ }^{54}$ and Constantinople ${ }^{55}$, should be considered a crucial feature of the working of the ancient economy ${ }^{56}$. The same phenomenon of interlocking action radii explains the contrast between, for instance, the regional origin of table wares and the empire-wide amphora supplies at Mons Claudianus ${ }^{57}$.

Archaeozoological evidence for the transport and trade of preserved or fresh fish along the Nile is absent, since the ichthyofauna is more or less similar throughout the Egyptian part of the Nile ${ }^{58}$. Some species, such as Oreochromis aureus, are

52. YOUNG, $2001: 27-89$.

53. KARAGIORGOU, 2001.

54. ELAIGNE, 1998.

55. SODINI, 2000.

56. FULFORD, 1987 ; TEMIN, 2001

57. TOMBER, 1996.

58. Cf. BOULENGER, 1907 : vii-xviii. 
typical for the Delta, but thus far no diagnostic criteria have been described to distinguish those fish from related species in the Nile (three other tilapia species in the case of Oreochromis aureus). Several ancient sources, mostly papyri datable between the third century BC and the Arab conquest, document fishing, fish, fish preparation and consumption and also contain some information on fish trade ${ }^{59}$. Dried fish formed an integral part of the diet of the Roman arny in Egypt and on occasion was even served to the emperor Caracalla. Gartm, on the other hand, may not have become popular before mid imperial times ${ }^{60}$. Fish trade is documented within Egypt, for example, within the nome of Arsinoë, or, on a much larger scale, between the Fayum and Alexandria. Trade in fish was clearly important enough for the Roman government in Egypt to exact taxes on the transport of fish products at its regional customs depots. In this context, the early imperial transport company directed by Nicanor should be mentioned. It specialized in organizing caravans into the Eastern Desert in order to supply individuals at Myos Hormos and Berenike with assorted daily items, food and drink ${ }^{61}$. Such private initiatives mushroomed with the opportunities offered by the Red Sea trade. As detailed and lively as the papyrological data may be, it is unfortunate that very few of these inferences can be verified in the Egyptian archaeological record. No fish preparation units have yet been studied, nor is it possible to link specific types of amphorae or other containers to the vessels that were particularly associated with transporting fish products, as attested in the papyri.

\section{TO THE SINAI AND}

\section{THE SYRO-PALESTINIAN AREA}

Remains of Nilotic fish have been discovered outside of the African part of Egypt on many archaeological sites ranging in time from the Chalcolithic to the Crusader period (tables 3 and 4). The only find known from the Chalcolithic is from Tel Katif, suggesting that the trade of fish was not yet well developed. Other faunal indicators of contact between Palestine and Egypt in that period are shells of the Nile bivalve Spathopsis mbens ${ }^{62}$, which are found at a growing number of sites including Teleilat Ghassul, Gerar, Gilat, Hor-

59. CURTIS, $1991: 131-141$

60. DREXHAGE, 1993.

61. YOUNG, $2001: 59$.

62. Mainly described under their synonym Aspatharia mbens. vat Rakik, Peqiin, Ben Shemen, Tell Abu-Matar and Horvat Beter $^{63}$. These shells and a limited number of other finds attest only to sporadic contact and "down-the-line" trade between Egypt and the southern Levant (Ghassul-Beer Sheva cultures). However, new finds provide evidence for the presence of Levantine immigrants in Egypt based on diagnostic pottery (at Buto), and architectural remains (at Maadi), suggesting more intensive contact with the Delta than the few imports in both regions imply. Nevertheless no evidence for systematic long-distance trading relations is apparent. The land route over northern Sinai is part of the Chalcolithic settlement pattern at this time with only limited evidence of Egyptian goods ${ }^{64}$. Whether the Lates bones from Tel Katif, located along the coast at the end of the land route, where Mediterranean fish have also been found, indicate maritime activities that included forays to Egypt remains to be explored as more of the coastal sites of this period are examined 65 .

Over the course of the Bronze Age in the Levant, it can be observed that finds of Nilotic fish are extremely rare in the Early Bronze Age, more frequent during the Middle Bronze Age, and their abundance increases during the Late Bronze Age, but they are never as extensive as in the Iron Age. At the beginning of the EBA sustained contacts with Egypt begin to be developed. The North Sinai land route is utilized by the Lower Egyptians (Maadi), apparently to insure the import of copper, along with other more easily traceable objects and raw materials. Maritime trade at this early time is suggested by the discovery of a jar of Nilotic clay with parallels at Maadi filled with Spathopsis shells $700 \mathrm{~m}$ off shore at Atlit Bay 66 . Similar shells have been found associated with the initial EBI phases at the coastal site of Afridar, and the recovery of Nile fish at the same site from the same phase may be a further indication of international maritime activities that will become more intensive in following periods ${ }^{67}$. In light of the notable colonial expansion of the Egypt state.into Canaan in the later part of the $\mathrm{EBI}^{68}$, the lack of fish remains is remarkable, although shells continue to be found especially in areas of sustained Egyptian interest both inland and on the coast ${ }^{69}$. The reason for this lack may be partly due to the apparent dominance of the overland route to move the goods (which in

63. REESE et al., 1986; BAR-YOSEF MAYER, 2002.

64. LEVY and VAN DEN BRINK, 2002 : 18 ; MIROSCHEDJ, 2002 ; YEKU. TIELI, 2002.

65. MARCUS, 2002.

66. SHARVIT et al., 2002.

67. See also GOPHNA, 2002 ; MARCUS, 2002

68. LEVY and VAN DEN BRINK, 2002.

69. BAR-YOSEF MAYER, 2002. 
Table 3 : Evidence for fish trade in sites from the Sinai, Israel and Palestine.

\begin{tabular}{|c|c|c|c|c|}
\hline SITE & PERIOD & TAXON & ORIGIN IN PUBLICATION & $\begin{array}{l}\text { NEWV IN'TER- } \\
\text { PRETATION }\end{array}$ \\
\hline Afridar & Early Bronze Age I & Lates niloticus (Nile perch) & Nile $(350 \mathrm{~km})$ & \\
\hline Apolonia $^{\mathrm{b}}$ & Hellenistic & Lates niloticus (Nile perch) & Nile $(400 \mathrm{~km})$ & \\
\hline Ashdod $\mathrm{c}$ & Early Iron Age & Lates niloticus (Nile perch) & Nile $(360 \mathrm{~km})$ & \\
\hline Beer Shevad $^{d}$ & Iron Age II & Lates niloticus (Nile perch) & Nile $(350 \mathrm{~km})$ & \\
\hline \multirow[t]{3}{*}{ Caesarea } & Byzantine & $\begin{array}{l}\text { Clarias gariepimus (catfish) } \\
\text { Tilapini (tilapia) }\end{array}$ & $\begin{array}{l}\text { local river (Taninim } 0 \mathrm{~km}) ; \\
\text { Lake Galilee }(70 \mathrm{~km}) \\
\text { local river }(\text { Taninim } 0 \mathrm{~km}) ; \\
\text { Lake Galilee }(70 \mathrm{~km})\end{array}$ & \\
\hline & Early lslamic & $\begin{array}{l}\text { Lates niloticus (Nile perch) } \\
\text { Bagrus sp. (catfish) } \\
\text { Clarias gariepinus (catfish) } \\
\text { Tilapiini (tilapia) } \\
\text { Barbus sp. (barbel) }\end{array}$ & $\begin{array}{l}\text { Nile }(400 \mathrm{~km}) \\
\text { Nile }(400 \mathrm{~km}) \\
\text { local river }(\text { Taninim } 0 \mathrm{~km}) \\
\text { Lake Galilee }(70 \mathrm{~km}) \\
\text { local river }(\text { Taninim } 0 \mathrm{~km}) \\
\text { Lake Galilee }(70 \mathrm{~km}) \\
\text { Lake Galilee }(70 \mathrm{~km}) \\
\end{array}$ & \\
\hline & Crisader period & $\begin{array}{l}\text { Lates niloticus (Nile perch) } \\
\text { Clarias gariepinus (catfist) } \\
\text { Tilapini (tilapia) }\end{array}$ & $\begin{array}{l}\text { Nile }(400 \mathrm{~km}) \\
\text { local river }(\text { Taninim } 0 \mathrm{~km}) \\
\text { Lake Galilee }(70 \mathrm{~km}) \\
\text { local river }(\text { Taninim } 0 \mathrm{~km}) \\
\text { Lake Galilee }(70 \mathrm{~km})\end{array}$ & \\
\hline Ein Gedif & Roman/Byzantine & $\begin{array}{l}\text { Clarias gariepimus (catfish) } \\
\text { Tilapini (tilapia) } \\
\text { Spartus auratus (gilthead seabream) } \\
\text { Scartus sp. (parrotfish) }\end{array}$ & \begin{tabular}{|l|} 
Jordan river $(25 \mathrm{~km})$ \\
Jordan river $(25 \mathrm{~km})$ \\
Mediterranean $(80 \mathrm{~km})$ \\
Red Sea $(220 \mathrm{~km})$ \\
\end{tabular} & \\
\hline Ein Hagits & Early Iron Age & $\begin{array}{l}\text { Lates niloticus (Nile perch) } \\
\text { Pagrus sp. (seabream) }\end{array}$ & \begin{tabular}{|l|} 
Nile $(450 \mathrm{~km})$ \\
Mediterranean $(8 \mathrm{~km})$ \\
\end{tabular} & \\
\hline En Boqeq & Late Roman-Early Byzantine & $\begin{array}{l}\text { Cyprinidae (barbels) } \\
\text { Clarias gariepinus (catfish) } \\
\text { Tilapiini (tilapia) } \\
\\
\text { Lethrinus sp. (emperor) } \\
\text { Scanus sp. (parrotfish) } \\
\text { Epinephelus aeneus (white grouper) } \\
\text { Dentex sp. (dentex) } \\
\text { Argyrosomus regius (meagre) } \\
\text { Serranidae (groupers) } \\
\text { Mugilidae (mullets) } \\
\end{array}$ & $\begin{array}{l}\text { Levant (Jordan R. } 70 \mathrm{~km} \text {; } \\
\text { coastal rivers } 100 \mathrm{~km}) \\
\text { Levant (Jordan R. } 70 \mathrm{~km} \text {; } \\
\text { coastal rivers } 100 \mathrm{~km}) \\
\text { Levant (Jordan R. } 70 \mathrm{~km} \text {; } \\
\text { coastal rivers } 100 \mathrm{~km}) \\
\text { Red Sea }(190 \mathrm{~km}) \\
\text { Red Sea }(190 \mathrm{~km}) \\
\text { Mediterranean }(90 \mathrm{~km}) \\
\text { Mediterranean }(90 \mathrm{~km}) \\
\text { Mediterranean }(90 \mathrm{~km}) \\
\text { Mediterranean } / \text { Red Sea } \\
\text { Mediterranean/Red Sea } \\
\end{array}$ & $\begin{array}{l}\text { Levant or Nile } \\
\text { Levant or Nile } \\
\text { Levant or Nile }\end{array}$ \\
\hline Gamla $^{i}$ & Early Roman $\left(1^{\text {st }}\right.$ c. AD) & $\begin{array}{l}\text { Clarias gariepimus (catfish) } \\
\text { Cyprinidae (barbels) } \\
\text { Argyrosomus regius (meagre) }\end{array}$ & $\begin{array}{l}\text { Lake Galilee }(10 \mathrm{~km}) \\
\text { Lake Galilee }(10 \mathrm{~km}) \\
\text { Mediterranean }(65 \mathrm{~km}) \\
\end{array}$ & \\
\hline Hanuvitj & Late Bronze Age & Lates niloticus (Nile perch) & Nile $(100 \mathrm{~km})$ & \\
\hline \multirow[t]{2}{*}{ Hatoulak } & Natufian & $\begin{array}{l}\text { Serranidae (groupers) } \\
\text { Spartus aurata (gilthead seabream) } \\
\text { Argyrosomus regius (meagre) } \\
\text { Mugil cephalus (mullet) } \\
\end{array}$ & $\begin{array}{l}\text { Mediterranean }(30 \mathrm{~km}) \\
\text { Mediterranean }(30 \mathrm{~km}) \\
\text { Mediterranean }(30 \mathrm{~km}) \\
\text { Mediterranean }(30 \mathrm{~km})\end{array}$ & \\
\hline & PPNA & $\begin{array}{l}\text { Tilapia cf. zillii (tilapia) } \\
\text { Epinephelus spp. (groupers) } \\
\text { Dicentrarchus labrax (sea bass) } \\
\text { Seriola dumerili (amberjack) } \\
\text { Sparus aurata (gilthead seabream) }\end{array}$ & $\begin{array}{l}\text { coastal rivers (Yarkon at } 30 \mathrm{~km}) \\
\text { Mediterranean }(30 \mathrm{~km}) \\
\text { Mediterranean }(30 \mathrm{~km}) \\
\text { Mediterranean }(30 \mathrm{~km}) \\
\text { Mediterranean }(30 \mathrm{~km})\end{array}$ & \\
\hline
\end{tabular}


Table 3 : Evidence for fish trade in sites from the Sinai, Israel and Palestine. (continued)

\begin{tabular}{|c|c|c|c|c|}
\hline SITE & PERIOD & TAXON & ORIGIN IN PUBLICATION & $\begin{array}{l}\text { NEIV INTER- } \\
\text { PRETATION }\end{array}$ \\
\hline Hatoula & PPNA & $\begin{array}{l}\text { Pagrus sp. (seabream) } \\
\text { Dentex ?dentex (dentex) } \\
\text { Diplodus ?sargus (seabream) } \\
\text { Lithognathus mormynus (striped } \\
\text { seabream) } \\
\text { Pagellus ?erythrimus (common pan- } \\
\text { dora) } \\
\text { Sciaena tmbra (brown meagre) } \\
\text { Mugil cephalus (mullet) } \\
\text { Liza ramada (mullet) } \\
\text { Euthymus alletteratus (little tuny) }\end{array}$ & $\begin{array}{l}\text { Mediterranean }(30 \mathrm{~km}) \\
\text { Mediterranean }(30 \mathrm{~km}) \\
\text { Mediterranean }(30 \mathrm{~km}) \\
\text { Mediterranean }(30 \mathrm{~km}) \\
\text { Mediterranean }(30 \mathrm{~km}) \\
\text { Mediterranean }(30 \mathrm{~km}) \\
\text { Mediterranean }(30 \mathrm{~km}) \\
\text { Mediterranean }(30 \mathrm{~km}) \\
\text { Mediterranean }(30 \mathrm{~km})\end{array}$ & \\
\hline Horvat Karkur! & Byzantine (5 $5^{\text {th }}-7^{\text {th }}$ c. AD) & $\begin{array}{l}\text { Sparisoma cretense (parrotfish) } \\
\text { Argyrosomus regius (meagre) } \\
\text { Scarus sp. (parrotfish) } \\
\text { Epinephelus sp. (grouper) } \\
\text { Liza sp. (mullet) } \\
\text { Tilapia sp. (tilapia) } \\
\text { Barbus sp. (barbel) } \\
\text { Clarias gariepimus (catfish) } \\
\text { Lates niloticus (Nile perch) }\end{array}$ & $\begin{array}{l}\text { Mediterranean }(40 \mathrm{~km}) \\
\text { Mediterranean }(40 \mathrm{~km}) \\
\text { Red Sea }(200 \mathrm{~km}) \\
\text { cf. Red Sea }(200 \mathrm{~km}) \\
\text { cf. Red Sea }(200 \mathrm{~km}) \\
\text { cf. Jordan valley }(180 \mathrm{~km}) \\
\text { cf. Jordan valley }(180 \mathrm{~km}) \\
\text { cf. Jordan valley }(180 \mathrm{~km}) \\
\text { cf. Nile }(350 \mathrm{~km})\end{array}$ & $\begin{array}{l}\text { Nile or Levant } \\
\text { Nile or Levant } \\
\text { Nile }\end{array}$ \\
\hline Horvat Rosh Zayitn & Iron Age II & $\begin{array}{l}\text { Lates niloticus (Nile perch) } \\
\text { Clarias gariepinus (catfish) } \\
\text { at least } 4 \text { marine taxa }\end{array}$ & $\begin{array}{l}\text { Nile }(450 \mathrm{~km}) \\
\text { coastal rivers (Kishon at } 15 \mathrm{~km}) \\
\text { Mediterranean }(15 \mathrm{~km})\end{array}$ & \\
\hline \multirow[t]{2}{*}{ Jaffa ${ }^{n}$} & Hellenistic ( $2^{\text {nd }}-1^{\text {st }}$ c. BC) & Lates niloticus (Nile perch) & Nile $(380 \mathrm{~km})$ & \\
\hline & Crusader period & Scarus sp. (parrotfish) & Red Sea $(250 \mathrm{~km})$ & \\
\hline \multirow[t]{2}{*}{$\begin{array}{l}\text { Jerusalem, } \\
\text { Ammenian monastery }\end{array}$} & Byzantine & $\begin{array}{l}\text { Clarias gariepimus (catfish) } \\
\text { Scartus sp. (parrotfish) }\end{array}$ & $\begin{array}{l}\text { Levant (Jordan R. } 32 \mathrm{~km} \text {; coastal } \\
\text { rivers } 45 \mathrm{~km}) \\
\text { Red Sea }(240 \mathrm{~km})\end{array}$ & \\
\hline & Early Islamic & $\begin{array}{l}\text { Lates niloticus (Nile perch) } \\
\text { Clarias gariepims (catfish) } \\
\text { Tilapiini (tilapia) } \\
\text { at least } 4 \text { marine taxa } \\
\text { Scartus } \text { sp. (parrotfish) }\end{array}$ & $\begin{array}{l}\text { Nile }(410 \mathrm{~km}) \\
\text { Levant (Jordan R. } 32 \mathrm{~km} \text {; coastal } \\
\text { rivers } 45 \mathrm{~km}) \\
\text { Levant (Jordan R. } 32 \mathrm{~km} \text {; coastal } \\
\text { rivers } 45 \mathrm{~km}) \\
\text { Mediterranean }(50 \mathrm{~km}) \\
\text { Red Sea }(240 \mathrm{~km})\end{array}$ & \\
\hline \multirow[t]{3}{*}{$\begin{array}{l}\text { Jenusalem, City of } \\
\text { Davidp }\end{array}$} & Middle Bronze Age II & $\begin{array}{l}\text { Symodontis schall } \\
\text { Lates niloticts (Nile perch) } \\
\text { Tilapiini (tilapia) } \\
\\
\text { Epinephelus sp. (grouper) } \\
\text { Sparus atrata (gilthead seabream) } \\
\text { Mugilidae (mullets) }\end{array}$ & $\begin{array}{l}\text { Nile }(410 \mathrm{~km}) \\
\text { Nile or Levant } \\
\text { Levant (Jordan R. } 32 \mathrm{~km} ; \text { coastal } \\
\text { rivers } 45 \mathrm{~km}) \\
\text { Mediterranean }(50 \mathrm{~km}) \\
\text { Mediterranean }(50 \mathrm{~km}) \\
\text { Mediterranean }(50 \mathrm{~km})\end{array}$ & $\begin{array}{l}\text { Nile } \\
\text { Levant or Nile }\end{array}$ \\
\hline & Iron Age I & $\begin{array}{l}\text { Lates niloticus (Nile perch) } \\
\text { Epinephelus sp. (grouper) }\end{array}$ & $\begin{array}{l}\text { Nile or Levant } \\
\text { Mediterranean }(50 \mathrm{~km})\end{array}$ & Nile \\
\hline & Iron Age II & $\begin{array}{l}\text { Clarias gariepinus (catfish) } \\
\text { Tilapiini (tilapia) } \\
\text { Lafes niloticus (Nile perch) } \\
\text { Epinephelus sp. (grouper) } \\
\text { Argyrosomus regits (meagre) } \\
\text { Spartus aurata (gilthead seabream) } \\
\text { Mugilidae (mullets) }\end{array}$ & $\begin{array}{l}\text { Levant (Jordan R. } 32 \mathrm{~km} \text {; coastal } \\
\text { rivers } 45 \mathrm{~km}) \\
\text { Levant (Jordan R. } 32 \mathrm{~km} \text {; coastal } \\
\text { rivers } 45 \mathrm{~km}) \\
\text { Nile or Levant } \\
\text { Mediterranean }(50 \mathrm{~km}) \\
\text { Mediterranean }(50 \mathrm{~km}) \\
\text { Mediterranean }(50 \mathrm{~km}) \\
\text { Mediterranean }(50 \mathrm{~km})\end{array}$ & $\begin{array}{l}\text { Levant or Nile } \\
\text { Levant or Nile } \\
\text { Nile }\end{array}$ \\
\hline
\end{tabular}


Table 3 : Evidence for fish trade in sites from the Sinai, Israel and Palestine. (continued)

\begin{tabular}{|c|c|c|c|c|}
\hline SITE & PERIOD & TAXON & ORIGIN IN PUBLICATION & $\begin{array}{l}\text { NEW INTER- } \\
\text { PRETATION }\end{array}$ \\
\hline \multirow{4}{*}{$\begin{array}{l}\text { Jerusalem, City of } \\
\text { David }\end{array}$} & Persian & Sparts anrata (gilthead seabream) & Mediterranean $(50 \mathrm{~km})$ & \\
\hline & Hellenistic & Sparus aurata (gillhead seabream) & Mediterranean $(50 \mathrm{~km})$ & \\
\hline & Early Roman & $\begin{array}{l}\text { Lates niloticus (Nile perch) } \\
\text { Euthymus alletteratus (little tuny) }\end{array}$ & $\begin{array}{l}\text { Nile or Levant } \\
\text { Mediterranean }(50 \mathrm{~km})\end{array}$ & Nile \\
\hline & Byzantine & Elasmobranchii (rays and sharks) & Mediterranean $(50 \mathrm{~km})$ & \\
\hline Jentsalem, Ophelq & Iron Age II & $\begin{array}{l}\text { Clarias gariepints (catfish) } \\
\text { Tilapiini (tilapia) } \\
\text { Lates niloticus (Nile perch) } \\
\text { Epinephehts sp. (grouper) } \\
\text { Argyrosomus regitus (meagre) } \\
\text { Sparus aurata (gilthead seabream) } \\
\text { Pagrus pagrus (common seabream) } \\
\text { Mugilidae (mullets) }\end{array}$ & $\begin{array}{l}\text { Levant (Jordan R. } 32 \mathrm{~km} \text {; coastal } \\
\text { rivers } 45 \mathrm{~km}) \\
\text { Levant (Jordan R. } 32 \mathrm{~km} \text {; constal } \\
\text { rivers } 45 \mathrm{~km}) \\
\text { Nile or Levant } \\
\text { Mediterranean }(50 \mathrm{~km}) \\
\text { Mediterranean }(50 \mathrm{~km}) \\
\text { Mediterranean }(50 \mathrm{~km}) \\
\text { Mediterranean }(50 \mathrm{~km}) \\
\text { Mediterranean }(50 \mathrm{~km})\end{array}$ & $\begin{array}{l}\text { Levant or Nile } \\
\text { Levant or Nile } \\
\text { Nile }\end{array}$ \\
\hline Kuntillet 'Ajrud' & Iron Age Il & $\begin{array}{l}\text { Lates niloticus (Nile perch) } \\
\text { Spartus annata (gilthead seabream) } \\
\text { Epinephelus sp. (grouper) }\end{array}$ & $\begin{array}{l}\text { Nile }(330 \mathrm{~km}) \\
\text { Mediterranean }(120 \mathrm{~km}) \\
\text { Mediterranean/Red Sea }\end{array}$ & \\
\hline \multirow[t]{4}{*}{ Lachishs } & Middle Bronze Age & $\begin{array}{l}\text { Lates niloticus (Nile perch) } \\
\text { Claritus gariepimus (catfish) } \\
\text { Tilapiini (tilapia) } \\
\text { at least } 6 \text { marine taxa }\end{array}$ & $\begin{array}{l}\text { Nile }(380 \mathrm{~km}) \\
\text { coastal river }(60 \mathrm{~km}), \text { L. Galilee } \\
(150 \mathrm{~km}) \text {, Jordan } \mathrm{R} .(80 \mathrm{~km}) \\
\text { coastal river }(60 \mathrm{~km}), \text { L. Galilee } \\
\text { (150 km), Jordan } \mathrm{R} .(80 \mathrm{~km}) \\
\text { Mediterranean }(30 \mathrm{~km})\end{array}$ & \\
\hline & Late Bronze Age III & $\begin{array}{l}\text { Lates niloticus (Nile perch) } \\
\text { Bagrus sp. (catfish) } \\
\text { Distichodus sp. } \\
\text { Mormyridae (elephant-snout fish) } \\
\text { Clarias gariepinus (catfish) } \\
\text { Tilapiini (tilapia) } \\
\text { Barbus sp. (barbel) } \\
\text { at least } 7 \text { marine taxa }\end{array}$ & $\begin{array}{l}\text { Nile }(380 \mathrm{~km}) \\
\text { Nile }(380 \mathrm{~km}) \\
\text { Nile }(380 \mathrm{~km}) \\
\text { Nile }(380 \mathrm{~km}) \\
\text { coastal river }(60 \mathrm{~km}), \text { L. Galilee } \\
\text { (150km), Jordan R. }(80 \mathrm{~km}), \text { Nile } \\
\text { coastal river }(60 \mathrm{~km}), \text { L. Galilee } \\
(150 \mathrm{~km}), \text { Jordan R. }(80 \mathrm{~km}), \text { Nile } \\
\text { L. Galilee }(150 \mathrm{~km}), \text { Jordan R. } \\
(80 \mathrm{~km}), \text { Nile } \\
\text { Mediterranean }(30 \mathrm{~km})\end{array}$ & \\
\hline & Iron Age II & $\begin{array}{l}\text { Lates niloticus (Nile perch) } \\
\text { Clarias gariepinus (catfish) } \\
\text { at least } 6 \text { marine taxa }\end{array}$ & $\begin{array}{l}\text { Nile }(380 \mathrm{~km}) \\
\text { coastal river }(60 \mathrm{~km}), \text { L. Galilee } \\
(150 \mathrm{~km}), \text { Jordan } R .(80 \mathrm{~km}) \\
\text { Mediterranean }(30 \mathrm{~km})\end{array}$ & \\
\hline & Persian & Clarias gariepimus (catfísh) & $\begin{array}{l}\text { coastal river }(60 \mathrm{~km}), \mathrm{L} . \text { Galilee } \\
(150 \mathrm{~km}) \text {, Jordan } \mathrm{R} .(80 \mathrm{~km})\end{array}$ & \\
\hline Masadat & Early Roman (1st c. AD) & $\begin{array}{l}\text { Sardinella awrita (round sardinella) } \\
\text { Engraulis encrasicolus (anchovy) } \\
\text { at least } 4 \text { marine taxa }\end{array}$ & $\begin{array}{l}\text { Western Mediterranean ; Spain } \\
\text { Western Mediterranean ; Spain } \\
\text { Mediterranean }(80 \mathrm{~km})\end{array}$ & \\
\hline \multirow[t]{3}{*}{ Megiddo } & Early Bronze Age & at least 3 marine taxa & Mediterranean (25 km) & \\
\hline & Middle Bronze Age & Lates niloticus (Nile perch) & Nile $(430 \mathrm{~km})$ & \\
\hline & Late Bronze Age & $\begin{array}{l}\text { Lates niloticus (Nile perch) } \\
\text { Clarias gariepinus (catfish) } \\
\text { at least } 3 \text { marine taxa }\end{array}$ & $\begin{array}{l}\text { Nile }(430 \mathrm{~km}) \\
\text { coastal rivers (Taninim at } 25 \mathrm{~km}) \\
\text { Mediterranean }(25 \mathrm{~km})\end{array}$ & \\
\hline
\end{tabular}


Table 3 : Evidence for fish trade in sites from the Sinai, Israel and Palestine. (continued)

\begin{tabular}{|c|c|c|c|c|}
\hline SITE & PERIOD & TAXON & ORIGIN IN PUBLICATION & $\begin{array}{l}\text { NEIV INTER- } \\
\text { PRETATION }\end{array}$ \\
\hline \multirow[t]{2}{*}{ Megiddo } & Iron Age II & $\begin{array}{l}\text { Lates niloticus (Nile perch) } \\
\text { Clarias gariepinus (catfish) } \\
\text { Tilapiini (tilapia) } \\
\text { at least } 6 \text { marine taxa }\end{array}$ & $\begin{array}{l}\text { Nite }(430 \mathrm{~km}) \\
\text { coastal rivers (Taninim at } 25 \mathrm{~km}) \\
\text { coastal rivers (Taninim at } 25 \mathrm{~km}) \\
\text { Mediterranean }(25 \mathrm{~km})\end{array}$ & \\
\hline & Roman & Serranidae (groupers) & Mediterranean $(25 \mathrm{~km})$ & \\
\hline Neve Yarak" & Late Bronze Age & Lates niloticus (Nile perch) & Nile $(400 \mathrm{~km})$ & \\
\hline Qarantal'w & Roman & $\begin{array}{l}\text { Scarus sp. (parrotfish) } \\
\text { Clarias gariepinus (cattish) }\end{array}$ & $\begin{array}{l}\text { Red Sea }(260 \mathrm{~km}) \\
\text { local river (Jordan R. } 8 \mathrm{~km})\end{array}$ & \\
\hline Sasa $^{x}$ & Middle Bronze Age & $\begin{array}{l}\text { Clarias gariepinus (catfish) } \\
\text { Lates niloticus (Nile perch)y }\end{array}$ & $\begin{array}{l}\text { Levant (Jordan R. } 25 \mathrm{~km} \text {; coastal } \\
\text { rivers } 20 \mathrm{~km}) \text { or Nile }(470 \mathrm{~km}) \\
\text { Nile }(470 \mathrm{~km})\end{array}$ & \\
\hline Tamara $^{\mathrm{z}}$ & Late Roman-Early Byzantine & $\begin{array}{l}\text { Clarias gariepinus (catfish) } \\
\text { Tilapini (tilapia) } \\
\text { Lethrinus sp. (emperor) } \\
\text { Scarus sp. (parrotfish) } \\
\text { Epinephelus aeneus (white grouper) } \\
\text { Dentex sp. (dentex) } \\
\text { Sparus aurata (gitthead seabream) } \\
\text { Sarda sarta (bonito) } \\
\text { Argyrosomus regitus (meagre) } \\
\text { Scorpaena sp. (scorpion fish) } \\
\text { Serranidae (groupers) } \\
\text { Mugilidae (mullets) } \\
\text { Labrus sp. (wrass) } \\
\text { Atxis sp. } \\
\text { Euthymmis spp. (little tuny) }\end{array}$ & $\begin{array}{l}\text { Levant (Jordan R. } 90 \mathrm{~km} \text {; coastal } \\
\text { rivers } 100 \mathrm{~km}) \\
\text { Levant }(\mathrm{Jordan} \text { R. } 90 \mathrm{~km} \text {; coastal } \\
\text { rivers } 100 \mathrm{~km}) \\
\text { Red Sea }(170 \mathrm{~km}) \\
\text { Red Sea }(170 \mathrm{~km}) \\
\text { Mediterranean }(90 \mathrm{~km}) \\
\text { Mediterranean }(90 \mathrm{~km}) \\
\text { Mediterranean }(90 \mathrm{~km}) \\
\text { Mediterranean }(90 \mathrm{~km}) \\
\text { Mediterranean }(90 \mathrm{~km}) \\
\text { Mediterranean } / \text { Red Sea } \\
\text { Mediterranean } / \text { Red Sea } \\
\text { Mediterranean/Red Sea } \\
\text { Mediterranean } / \text { Red Sea } \\
\text { Mediterranean } / \text { Red Sea } \\
\text { Mediterranean } / \text { Red Sea }\end{array}$ & $\begin{array}{l}\text { Levant or Nile } \\
\text { Levant or Nile } \\
\text { Mediterranean } \\
\text { Red Sea }\end{array}$ \\
\hline Tel Abu-Hawam ${ }^{23}$ & Late Bronze Age & Lates niloticus (Nile perch) & Nile or Levant & Nile $(450 \mathrm{~km})$ \\
\hline \multirow[t]{6}{*}{ Tel Akko ab } & Late Bronze Age & $\begin{array}{l}\text { Lates niloticus (Nile perch) } \\
\text { Clarias gariepimus (catfish) } \\
\text { Tilapini (tilapia) } \\
\end{array}$ & $\begin{array}{l}\text { Nile or Levant } \\
\text { Na'aman River }(0 \mathrm{~km}) \\
\text { Na'aman River }(0 \mathrm{~km}) \\
\end{array}$ & Nile $(460 \mathrm{kn})$ \\
\hline & Iron Age I & Lates niloticus (Nile perch) & Nile or Levant & Nile \\
\hline & Iron Age II & Lates niloticus (Nile perch) & Nile or Levant & Nile \\
\hline & Iron Age & Clarias gariepinus (catfish) & Na'aman River $(0 \mathrm{~km})$ & \\
\hline & Persian & $\begin{array}{l}\text { Lates niloticus (Nile perch) } \\
\text { Synodontis sp. (catfish) } \\
\text { Tilapini (tilapia) } \\
\text { Clarias gariepinus (catfish) }\end{array}$ & $\begin{array}{l}\text { Nile or Levant } \\
\text { Nile }(460 \mathrm{~km}) \\
\text { Na'aman River }(0 \mathrm{~km}) \\
\text { Na'aman River }(0 \mathrm{~km}) \\
\end{array}$ & Nile \\
\hline & Hellenistic & Lates niloticus (Nile perch) & Nile $(460 \mathrm{~km})$ & \\
\hline \multirow[t]{3}{*}{ Tel Ashqelon ${ }^{\text {ac }}$} & Late Bronze Age/Iron Age I & $\begin{array}{l}\text { Lates niloticus (Nile perch) } \\
\text { Tilapini (tilapia) }\end{array}$ & $\begin{array}{l}\text { Nile or Levant } \\
\text { coastal rivers (Yarkon at } 50 \mathrm{~km} \text { ) }\end{array}$ & Nile \\
\hline & Iron Age II & $\begin{array}{l}\text { Lates niloticus } \text { (Nile perch) } \\
\text { Bagrus } \text { sp. (catfish) } \\
\text { Synodonis sp. (catfish) } \\
\text { Clarias gariepinus (catfish) } \\
\text { Tilapiini (tilapia) } \\
\text { Cyprinidae (barbels) } \\
\text { Scarus sp. (parrotfish) } \\
\end{array}$ & $\begin{array}{l}\text { Nile or Levant } \\
\text { Nile }(350 \mathrm{~km}) \\
\text { Nile }(350 \mathrm{~km}) \\
\text { coastal rivers (Yarkon at } 50 \mathrm{~km}) \\
\text { coastal rivers (Yarkon at } 50 \mathrm{~km}) \\
\text { coastal rivers (Yarkon at } 50 \mathrm{~km}) \\
\text { Red Sea }(225 \mathrm{~km}) \\
\end{array}$ & Nile \\
\hline & Persian & Lates niloticus (Nile perch) & Nile or Levant & Nile \\
\hline
\end{tabular}


Table 3 : Evidence for fish trade in sites from the Sinai, Israel and Palestine. (continued)

\begin{tabular}{|c|c|c|c|c|}
\hline SITE & PERIOD & TAXON & ORIGIN IN PUBLICATION & $\begin{array}{l}\text { NEWV INTER- } \\
\text { PRETATION }\end{array}$ \\
\hline \multirow[t]{3}{*}{ Tel Ashqeton } & Byzantine & $\begin{array}{l}\text { Lates niloticus (Nile perch) } \\
\text { Bagrus sp. (catfish) } \\
\text { Synodontis sp. (catfish) } \\
\text { Clarias gariepinus (catfish) } \\
\text { Tilapini (tilapia) } \\
\text { Cyprinidae (barbels) } \\
\text { Scartus sp. (parrotfish) } \\
\end{array}$ & $\begin{array}{l}\text { Nile }(350 \mathrm{~km}) \\
\text { Nile }(350 \mathrm{~km}) \\
\text { Nile }(350 \mathrm{~km}) \\
\text { coastal rivers (Yarkon at } 50 \mathrm{~km}) \\
\text { coastal rivers (Yarkon at } 50 \mathrm{~km}) \\
\text { coastal rivers (Yarkon at } 50 \mathrm{~km}) \\
\text { Red Sea }(225 \mathrm{~km}) \\
\end{array}$ & \\
\hline & Early Islamic & $\begin{array}{l}\text { Lates niloticus (Nile perch) } \\
\text { Clarias gariepinus (catfish) } \\
\text { Tilapiini (tilapia) } \\
\text { Scarus } \text { sp. (parrotfish) } \\
\end{array}$ & $\begin{array}{l}\text { Nile or Levant } \\
\text { coastal rivers (Yarkon at } 50 \mathrm{~km}) \\
\text { coastal rivers (Yarkon at } 50 \mathrm{~km}) \\
\text { Red Sea }(225 \mathrm{~km}) \\
\end{array}$ & Nile \\
\hline & Islamic/Crisader period & $\begin{array}{l}\text { Clarias gariepinus (catfish) } \\
\text { Scarus sp. (parrotfish) }\end{array}$ & $\begin{array}{l}\text { coastal rivers (Yarkon at } 50 \mathrm{~km}) \\
\text { Red Sea }(225 \mathrm{~km})\end{array}$ & \\
\hline \multirow[t]{5}{*}{ Tel Dor ad } & Middle Bronze Age & Lates niloticus (Nile perch) & Nile $(410 \mathrm{~km})$ & \\
\hline & Late Bronze Age & Lates niloticus (Nile perch) & Nile & \\
\hline & Iron Age Ia & $\begin{array}{l}\text { Bagrus sp. (catfish) } \\
\text { Clarias sp. (catfish) } \\
\end{array}$ & $\begin{array}{l}\text { Nile } \\
\text { coastal rivers (Taninim at } 8 \mathrm{~km} \text { ) }\end{array}$ & \\
\hline & Iron Age & Lates niloticus (Nile perch) & Nile & \\
\hline & \begin{tabular}{|l|} 
Persian \\
\end{tabular} & Lates niloticus (Nile perch) & Nile & \\
\hline Tel Gerisa ${ }^{\text {af }}$ & Iron Age II & $\begin{array}{l}\text { Lates niloticus (Nile perch) } \\
\text { Scarus sp. (parrotfish) }\end{array}$ & $\begin{array}{l}\text { Nile }(400 \mathrm{~km}) \text { or Levant } \\
\text { Red Sea }(280 \mathrm{~km})\end{array}$ & Nile \\
\hline \multirow[t]{2}{*}{ Tel Hamidah } & Iron Age II & $\begin{array}{l}\text { Lates niloticus (Nile perch) } \\
\text { Clarias gariepints (catfish) } \\
\text { at least } 3 \text { marine taxa }\end{array}$ & $\begin{array}{l}\text { Nile }(380 \mathrm{~km}) \\
\text { coastal rivers (Yarkon at } 20 \mathrm{~km}) \\
\text { Mediterranean }(20 \mathrm{~km})\end{array}$ & \\
\hline & Byzantine $\left(6^{\mathrm{th}-7^{\text {th }}} \mathrm{AD}\right)$ & Cichlidae & coastal rivers (Yarkon at $20 \mathrm{~km}$ ) & \\
\hline \multirow[t]{4}{*}{ Tel Harassimai } & Late Bronze Age Ila & $\begin{array}{l}\text { Lates niloticus (Nite perch) } \\
\text { at least } 4 \text { marine taxa }\end{array}$ & $\begin{array}{l}\text { Nile or Levant } \\
\text { Mediterranean }(20 \mathrm{~km})\end{array}$ & Nile \\
\hline & Iron Age II $\left(9^{\text {th }} \cdot 8^{\text {th }} \mathrm{BC}\right)$ & $\begin{array}{l}\text { Lates niloticus } \text { (Nile perch) } \\
\text { at least } 5 \text { marine taxa }\end{array}$ & $\begin{array}{l}\text { Nile }(380 \mathrm{~km}) \\
\text { Mediterranean }(20 \mathrm{~km}) \\
\end{array}$ & \\
\hline & Persian $\left(5^{\text {th }} 4^{\text {th }} B C\right)$ & $\begin{array}{l}\text { Lates niloticus (Nile perch) } \\
\text { at least } 3 \text { marine taxa }\end{array}$ & $\begin{array}{l}\text { Nile }(380 \mathrm{~km}) \\
\text { Mediterranean }(20 \mathrm{~km})\end{array}$ & \\
\hline & Byzantine $\left(4^{\text {th }}-7^{\text {th }}\right.$ AD) & at least 2 marine taxa & Mediterranean $(20 \mathrm{~km})$ & \\
\hline \multirow[t]{4}{*}{ Tel Kabriaj } & Neolithic & at least 2 marine taxa & Mediterranean $(5 \mathrm{~km})$ & \\
\hline & Early Bronze Age & at least 3 marine taxa & Mediterranean $(5 \mathrm{~km})$ & \\
\hline & Middle Bronze Age & $\begin{array}{l}\text { Lates niloticus (Nile perch) } \\
\text { at least } 5 \text { marine taxa } \\
\text { Tilapini (tilapia) } \\
\end{array}$ & $\begin{array}{l}\text { Nile }(470 \mathrm{~km}) \\
\text { Mediterranean }(5 \mathrm{~km}) \\
\text { coastal rivers }(1.4 \mathrm{~km}) \\
\end{array}$ & \\
\hline & Iron Age II & $\begin{array}{l}\text { Lales niloticus (Nile perch) } \\
\text { at least } 4 \text { marine taxa }\end{array}$ & $\begin{array}{l}\text { Nile }(470 \mathrm{~km}) \\
\text { Mediterranean }(5 \mathrm{~km})\end{array}$ & \\
\hline Tel Katifak & Chatcolithic & Lates niloticus (Nile perch) & Nile $(350 \mathrm{~km})$ or Levant & Nile \\
\hline Tel Malhatat & Roman/Byzantine & $\begin{array}{l}\text { Bagrus sp. (catfish) } \\
\text { Scarus sp. (parrotfish) }\end{array}$ & $\begin{array}{l}\text { Nile }(350 \mathrm{~km}) \\
\text { Red Sea }(190 \mathrm{~km}) \\
\end{array}$ & \\
\hline Tel Miqne $e^{a m}$ & Iron Age II & $\begin{array}{l}\text { Symodontis sp. (catfish) } \\
\text { Lates niloticus (Nile perch) } \\
\text { Citharhinidae } \\
\text { Bagrus sp. (catfish) } \\
\text { Clarias gariepinus (catfish) } \\
\text { Tilapini (tilapia) } \\
\text { at least } 10 \text { marine taxa }\end{array}$ & $\begin{array}{l}\text { Nile }(380 \mathrm{~km}) \\
\text { Nile }(380 \mathrm{~km}) \\
\text { Nile }(380 \mathrm{~km}) \\
\text { Nile }(380 \mathrm{~km}) \\
\text { coastal rivers (Yarkon at } 40 \mathrm{~km}) \\
\text { coastal rivers (Yarkon at } 40 \mathrm{~km}) \\
\text { Mediterranean }(25 \mathrm{~km}) \\
\end{array}$ & \\
\hline Tel-el-Wawayat ${ }^{\text {an }}$ & Middle Bronze Age & Lates niloticus (Nile perch) & Nile $(450 \mathrm{~km})$ & \\
\hline
\end{tabular}


Table 3 : Evidence for fish trade in sites from the Sinai, Israel and Palestine. (continued)

\begin{tabular}{|c|c|c|c|c|}
\hline SITE & PERIOD & TAXON & ORIGIN IN PUBLICATION & $\begin{array}{l}\text { NEWVINTER- } \\
\text { PRETATION }\end{array}$ \\
\hline \multirow[t]{2}{*}{ Tel-el-IVawayat } & Late Bronze Age & $\begin{array}{l}\text { Lates niloticus (Nile perch) } \\
\text { Sparidae (seabreams) }\end{array}$ & $\begin{array}{l}\text { Nile or Levant } \\
\text { Mediterranean }(20 \mathrm{~km})\end{array}$ & Nile \\
\hline & Iron Age I & Lates niloticts (Nile perch) & Nile $(450 \mathrm{~km})$ & \\
\hline Tell el-Hesiao & Iron Age II & $\begin{array}{l}\text { Clarias gariepimus (catfish) } \\
\text { Cichlidac } \\
\text { Lates niloticus (Nile perch) } \\
\text { Serranidae (groupers) }\end{array}$ & $\begin{array}{l}\text { Nile or coastal river (Yarkon at } \\
65 \mathrm{~km}) \\
\text { Nile or coastal river (Yarkon at } \\
65 \mathrm{~km}) \\
\text { Nile }(350 \mathrm{~km}) \\
\text { Mediterrnean }(25 \mathrm{~km}) \\
\end{array}$ & \\
\hline \multirow[t]{4}{*}{ Tell el-Oreme:ap } & Early Bronze Age & Clarias gratiepinms (catfish) & Lake of Galilee $(0 \mathrm{~km})$ & \\
\hline & $\begin{array}{l}\text { Middle Bronze Age/Late } \\
\text { Bronze Age }\end{array}$ & $\begin{array}{l}\text { Lates niloticus (Nite perch) } \\
\text { Clarias gariepinus (catfisl) }\end{array}$ & $\begin{array}{l}\text { Nile }(480 \mathrm{~km}) \\
\text { Lake of Galilee }(0 \mathrm{~km})\end{array}$ & \\
\hline & Iron Age I & $\begin{array}{l}\text { Tilapini (tilapia) } \\
\text { Clarias gariepimus (catfish) } \\
\text { Epinephelts sp. (grouper) }\end{array}$ & $\begin{array}{l}\text { Lake of Galilee }(0 \mathrm{~km}) \\
\text { Lake of Galilee }(0 \mathrm{~km}) \\
\text { Mediterranean }(45 \mathrm{~km})\end{array}$ & \\
\hline & Iron Age 11 & $\begin{array}{l}\text { Lates niloticus (Nile perch) } \\
\text { Clarias gaviepintus (catfish) } \\
\text { Tilapiini (tilapia) }\end{array}$ & $\begin{array}{l}\text { Levant } \\
\text { Lake of Galilee }(0 \mathrm{~km}) \\
\text { Lake of Galilee }(0 \mathrm{~km})\end{array}$ & $\begin{array}{l}\text { Nile } \\
\text { Levant or Nile } \\
\text { Levant or Nile }\end{array}$ \\
\hline \multirow[t]{2}{*}{ Tell Jeninaq } & Early Bronze Age & Epinephelus sp. (grouper) & Mediterranean $(38 \mathrm{~km})$ & \\
\hline & Byzantine & Lates niloticus (Nile perch) & Nile $(430 \mathrm{~km})$ & \\
\hline \multirow[t]{2}{*}{ Tell Qasilaar } & Iron Age I & Lates nilotictis (Nile perch) & Nile $(390 \mathrm{~km})$ & \\
\hline & Iron Age II & Lates niloticus (Nile perch) & Nile or Levant & Nile \\
\hline \multirow[t]{2}{*}{ Tell Rehovas } & Iron Age Ib & Lates niloticus (Nile perch) & Nile $(430 \mathrm{~km})$ & \\
\hline & Iron Age II & Lates niloticus (Nile perch) & Nile $(430 \mathrm{~km})$ & \\
\hline Timnat & Late Bronze Age to Iron Age I & $\begin{array}{l}\text { Clarias gariepinus (catfish) } \\
\text { Sparus aurata (gilthead seabream) } \\
\text { Argyrosomus tegitus (meagre) } \\
\text { Carcharhinidae (requiem sharks) }\end{array}$ & $\begin{array}{l}\text { Nile }(400 \mathrm{~km}) \\
\text { Mediterranean }(185 \mathrm{~km}) \\
\text { Mediterranean }(185 \mathrm{~km}) \\
\text { Mediterranean }(185 \mathrm{~km})\end{array}$ & \\
\hline Upper Zohar & Early Byzantine & 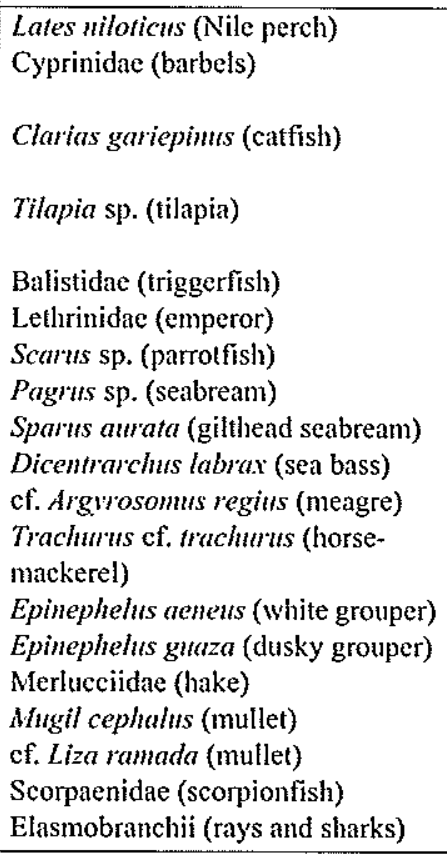 & $\begin{array}{l}\text { Nile or Levant } \\
\text { Levant (Jordan } 70 \mathrm{~km} \text {; coastal } \\
\text { rivers (Yarkon } 105 \mathrm{~km}) \text { ) } \\
\text { Levant (Jordan } 70 \mathrm{~km} \text {; coastal } \\
\text { rivers (Yarkon } 105 \mathrm{~km}) \text { ) } \\
\text { Levant (Jordan } 70 \mathrm{~km} \text {; coastal } \\
\text { rivers (Yarkon } 105 \mathrm{~km})) \\
\text { Red Sea }(190 \mathrm{~km}) \\
\text { Red Sea }(190 \mathrm{~km}) \\
\text { Red Sea }(190 \mathrm{~km}) \\
\text { Mediterranean }(80 \mathrm{~km}) \\
\text { Mediterranean }(80 \mathrm{~km}) \\
\text { Mediterranean }(80 \mathrm{~km}) \\
\text { Mediterranean }(80 \mathrm{~km}) \\
\text { Mediterranean }(80 \mathrm{~km}) \\
\\
\text { Mediterranean }(80 \mathrm{~km}) \\
\text { Mediterranean }(80 \mathrm{~km}) \\
\text { Mediterranean }(80 \mathrm{~km}) \\
\text { Mediterranean } / \text { Red Sea } \\
\text { Mediterranean } / \text { Red Sea } \\
\text { Mediterranean } / \text { Red Sea } \\
\text { Mediterranean/Red Sea } \\
\end{array}$ & $\begin{array}{l}\text { Nile } \\
\text { Nile or Levant } \\
\text { Nile or Levant } \\
\text { Nile or Levant } \\
\\
\text { Mediterranean' } \\
\text { Red Sea }\end{array}$ \\
\hline
\end{tabular}


Table 3 : Evidence for fsh trade in sites from the Sinai, Israel and Palestine. (continued)

\begin{tabular}{|c|c|c|c|c|}
\hline SITE & PERIOD & TAXON & ORIGIN IN PUBLICATION & $\begin{array}{l}\text { NEW INTER- } \\
\text { PRETATION }\end{array}$ \\
\hline \multirow[t]{3}{*}{ Yavneh-Yamªv } & Iron Age II & Lates nilotictss (Nile perch) & Nile $(370 \mathrm{~km})$ & \\
\hline & Hellenistic & Lates niloticus (Nile perch) & Nile $(370 \mathrm{~km})$ & \\
\hline & Early Islamic & Synodontis sp. (catfish) & Nile $(370 \mathrm{~km})$ & \\
\hline Zichrin $^{\text {aw }}$ & Early Byzantine & Lates niloticus (Nile perch) & Nile $(400 \mathrm{~km})$ & \\
\hline Zippori $\mathrm{jx}$ & Roman & $\begin{array}{l}\text { Bagrus sp. (catfish) } \\
\text { Clarias gariepimus (catfish) } \\
\text { Tilapini (tilapin) } \\
\text { at least } 5 \text { marine taxa }\end{array}$ & $\begin{array}{l}\text { Nite }(450 \mathrm{~km}) \\
\text { constal rivers (Kishon at } 25 \mathrm{~km}) \\
\text { coastal rivers (Kishon at } 25 \mathrm{~km}) \\
\text { Mediterranean }(25 \mathrm{~km})\end{array}$ & \\
\hline
\end{tabular}

a. LERNAU, unpublished.

b. Ihid.

c. MAHER, umpublished,

d. LERNAU, unpublished.

e. FRADKIN and LERNAU, in press.

f. LERNAU, in press.

g. LERNAU, unpublished.

h. LERNAU, 1986.

i. LERNAU, unpublished.

j. Ibiul.

k. LERNAU and LERNAU, 1994.

l. Lernau, 2004.

m. LERNAU, unpublished.

n. Ibid.

o. Ibid.

p. LERNAU and LERNAU, 1992.

q. LERNAU and LERNAU, 1989.

r. LERNAU, unpublished.

s. Ibid.

t. COTTON et al., 1996; LERNAU et al., 1996.

i. LERNAU, 2000

v. LERNAU, unpublished.

w. Ibid.

x. HORWITZ, 1987

y. LERNAU, unpublished.

z. LERNAU, 1986 aa. LERNAU, 1996.

ab. LERNAU, 1996 ; LERNAU, unpublished.

ac. LERNAU, 1986/87; LERNAU, 1996 ; LERNAU, unpublished.

ad. LERNAU, unpublished.

ae. BARTOSIEWICZ, pers, comm.

af. LERNAU, 1986/1987.

ag. SADE, pers. comm.

ah. LERNAU, unpublished.

ai. LERNAU, 1996 ; LERNAU, unpublished.

aj. LERNAU, unpublished.

ak. LERNAU, 1996.

al. LERNAU, unptiblisited.

am. bid.

an. LERNAU, 1996 ; LERNAU, unpublished.

ao. LERNAU, unpublished.

ap. ZIEGLER and BOESSNECK, 1990 ; MANHART and VON DEN DRIESCH, in press.

aq. AL ZAWAHRA, 1999.

ar. LERNAU, 1986/1987 ; LERNAU, unpublished.

as. LERNAU, unpublished.

at. LERNAU, 1988 .

au. LERNAU, 1995.

av. LERNAU, unpublished ; excavations under the direction of M. FISCHER (2002), archaeozoology by M. SADE.

aw. LERNAU, unpublished ; excavations under the direction of M. FISCHER (1989), archaeozoology by M. SADE.

ax. LERNAU, unpublished. addition to extant luxury items may have included gold and textiles) from Egypt to exchange for those things (copper, asphalt, olive oil and wine) Egypt desired. It is conceivable that during this period of state sponsored trade, fish, being an inexpensive commodity with a relatively short shelf life, were not considered worth the trouble of transporting for this purpose. Even when Egypt turns northward to Byblos and engages in maritime trade for bulk commodities, such as wood, which is well documented in EBII and $\mathrm{EBIII}^{70}$, anchorage points along the Levantine coast show no evidence of fish. Trade that continued with Canaan into the Old Kingdom was apparently conducted by royal emissaries, in direct contact with local heads of

70. MARCUS, 2002 and references cited there. state, who were exchanging prestige items for local produce. Literary evidence suggests this trade was undertaken using the overland route, with occasional military forays to insure compliance ${ }^{71}$. Alternative explanations for the lack of fish remains may, of course, also be related to the relatively few excavated Early Bronze Age sites and inadequate sampling techniques during excavation.

For the Middle Bronze Age, Nilotic fish remains are known from the coastal sites of Tel Dor and Tel Kabri, and from the inland sites of Lachish, Megiddo, Sasa, Tel-elWawayat and the City of David. At some of these sites, the fish remains are the only evidence for Egyptian imports of

71. REDFORD, 1992 ; MIROSCHEDJI, 2002. 
Table 4 : Evidence for fish trade in sites from Lebanon, Jordan and Syria.

\begin{tabular}{|c|c|c|c|c|}
\hline SITE & PERIOD & TAXON & ORIGIN IN PUBLICATION & $\begin{array}{l}\text { NEW INTER- } \\
\text { PRETATION }\end{array}$ \\
\hline \multicolumn{5}{|l|}{ Lebanon } \\
\hline \multirow[t]{2}{*}{ Sarepta ${ }^{a}$} & Late Bronze II & Lates niloticus (Nile perch) & Nile $(480 \mathrm{~km})$ & \\
\hline & Iron Age l & Lates niloticus (Nile perch) & Nile $(480 \mathrm{~km})$ & \\
\hline \multicolumn{5}{|l|}{ Jordan } \\
\hline \multirow[t]{2}{*}{ Tall al-'Umayrib } & $\begin{array}{l}\text { Late Bronze Age/Early Iron } \\
\text { Age }\end{array}$ & $\begin{array}{l}\text { Lates niloficus (Nile perch) } \\
\text { Cyprinidae (minnows) }\end{array}$ & $\begin{array}{l}\text { Nile }(450 \mathrm{~km}) \\
\text { Jordan river }(40 \mathrm{~km})\end{array}$ & \\
\hline & Iron Age I & Lates niloticus (Nile perch) & Nile $(450 \mathrm{~km})$ & \\
\hline \multirow[t]{4}{*}{ Tell Hesbanc } & Iron Age II & $\begin{array}{l}\text { Scaridae (parrotfish) } \\
\text { Spartus aurata (gilthead seabream) } \\
\text { Serranidae (groupers) }\end{array}$ & $\begin{array}{l}\text { Red Sea }(250 \mathrm{~km}) \\
\text { Mediterranean }(100 \mathrm{~km}) \\
\text { Medit.Red Sea }\end{array}$ & \\
\hline & Late Hellenistic & $\begin{array}{l}\text { Clarias gariepimus (catfish) } \\
\text { Scombridae (tumas, mackerels...) }\end{array}$ & $\begin{array}{l}\text { Jordan river }(25 \mathrm{~km}) \\
\text { Medit./Red Sea }\end{array}$ & \\
\hline & Early \& Late Roman & $\begin{array}{l}\text { Tilapiini (tilapia) } \\
\text { Clarias gariepimis (catfish) } \\
\text { Scaridae (parrotfish) } \\
\text { Argyrosomus regitus (meagre) } \\
\text { Scombridae (tunas, mackerels...) }\end{array}$ & $\begin{array}{l}\text { Jordan river }(25 \mathrm{~km}) \\
\text { Jordan river }(25 \mathrm{~km}) \\
\text { Red Sea }(250 \mathrm{~km}) \\
\text { Mediterranean }(100 \mathrm{~km}) \\
\text { Medit./Red Sea } \\
\end{array}$ & \\
\hline & Early \& Late Byzantine & $\begin{array}{l}\text { Tilapiini (tilapia) } \\
\text { Clarias garicpinus (catfish) } \\
\text { Scaridae (parrotfish) } \\
\text { Argyrosomus regius (meagre) } \\
\text { Scombridae (tunas, mackerels...) } \\
\text { Sparus aurata (gilthead seabream) }\end{array}$ & $\begin{array}{l}\text { Jordan river }(25 \mathrm{~km}) \\
\text { Jordan river }(25 \mathrm{~km}) \\
\text { Red Sea }(250 \mathrm{~km}) \\
\text { Mediterranean }(100 \mathrm{~km}) \\
\text { Medit./Red Sea } \\
\text { Mediterranean }(100 \mathrm{~km}) \\
\end{array}$ & \\
\hline \multirow[t]{2}{*}{ Petrad $^{d}$} & Nabataean & $\begin{array}{l}\text { Clarias gariepinus (catfish) } \\
\text { Carangidae (trevallies and jacks) } \\
\text { Haemulidae (grunts) } \\
\text { Lethrimus sp. (emperor) } \\
\text { Lutjamus sp. (snapper) } \\
\text { Scartus sp. (parrotfish) } \\
\text { Euthymmis sp. (little tuny) } \\
\text { Scomberomortis sp. } \\
\text { Epinephelus sp. (grouper) } \\
\text { Sparidae (seabream) } \\
\text { Sphyraena sp. (barracuda) }\end{array}$ & $\begin{array}{l}\text { Levant? (Jordan R. } 170 \mathrm{~km}) \\
\text { Red Sea }(100 \mathrm{~km}) \\
\text { Red Sea }(100 \mathrm{~km}) \\
\text { Red Sea }(100 \mathrm{~km}) \\
\text { Red Sea }(100 \mathrm{~km}) \\
\text { Red Sea }(100 \mathrm{~km}) \\
\text { Red Sea }(100 \mathrm{~km}) \\
\text { Red Sea }(100 \mathrm{~km}) \\
\text { Red Sea }(100 \mathrm{~km}) \\
\text { Red Sea }(100 \mathrm{~km}) \\
\text { Red Sea }(100 \mathrm{~km}) \\
\end{array}$ & $\begin{array}{l}\text { Levant or Nile } \\
(400 \mathrm{~km})\end{array}$ \\
\hline & Late Roman & $\begin{array}{l}\text { Labridae (wrasses) } \\
\text { Lethrinus sp. (emperor) } \\
\text { Scanus sp. (parrotfish) } \\
\text { Euthymus sp. (little tuny) } \\
\text { Epinephelus sp. (grouper) } \\
\text { Sigamus sp. (rabbitfish) } \\
\text { Argyrops spinifer (soldierbream) } \\
\text { Acanthopagrus sp. (seabream) }\end{array}$ & $\begin{array}{l}\text { Red Sea }(100 \mathrm{~km}) \\
\text { Red Sea }(100 \mathrm{~km}) \\
\text { Red Sea }(100 \mathrm{~km}) \\
\text { Red Sea }(100 \mathrm{~km}) \\
\text { Red Sea }(100 \mathrm{~km}) \\
\text { Red Sea }(100 \mathrm{~km}) \\
\text { Red Sea }(100 \mathrm{~km}) \\
\text { Red Sea }(100 \mathrm{~km})\end{array}$ & \\
\hline Petra ${ }^{\mathrm{c}}$ & Late Roman & Clipeiformes (sardines, anchovies...) & Red Sea $(100 \mathrm{~km})$ & \\
\hline \multicolumn{5}{|l|}{ Syria } \\
\hline Apamea ${ }^{f}$ & Early Byzantine (pre-613 AD) & Epinephelus cf. gttaza (grouper) & Mediterranean $(50 \mathrm{~km})$ & \\
\hline Palmyrag & $7^{\text {th }} \cdot 8^{\text {th }}$ cent. $\mathrm{AD}$ & Scaridae (parrotfish) & Red Sea $(650 \mathrm{~km})$ & \\
\hline
\end{tabular}

a. ROSE, 1994.

b. PETERS et al., 2002.

c. VON DEN DRIESCH and BOESSNECK, 1995 ; LEPIKSAAR, 1995.

d. DESSE-BERSET and STUDER, 1996. e. STUDER, 1994.

f. VAN NEER, 1984.

g. VAN NEER, unpublished. 
any type. After a period of political instability in Egypt in the First Intermediate Period and urban collapse in the Southern Levant, trade between Egypt and its eastern neighbours picks up again. During the strong central government of Egypt's $12^{\text {th }}$ Dynasty, Byblos remains a major trading partner for timber via maritime routes along the coast. Commercial relations with towns along the Canaanite coast probably began when provisioning boats on the Byblos run, but contact with Egypt alone was not the prime motivation for the maritime orientation of coastal developments in the Levant, as trade was undertaken in a more international arena, with the Aegean now playing a role. Trade with Egypt during the $12^{\text {th }}$ Dynasty-MBIIA is attested both overland, on an ad hoc basis with nomadic traders as depicted in the tomb of Khnumhotep at Beni Hassan, and also apparently by sea, to judge from the extremely small amount of Canaanite pottery (less than $1 \%$ ) found in the houses of the capital at Lisht ${ }^{72}$. State relations also apparently continued along the Old Kingdom model with royal emissaries in direct contact with local heads of state, and occasional military forays to insure compliance, during which war booty and human captives were obtained, sometimes on a vast scale ${ }^{73}$. The value of the evidence for the type of contact derived from the Egyptian materials found in Canaan at this time is a matter of dispute ${ }^{74}$, however, the Egyptian Execration texts indicate growing knowledge of the Levantine urban landscape and the various routes throughout the region.

In the $13^{\text {th }}$ Dynasty-MBIIB, the nature of trade relations changes as the overwhelmingly Asiatic town of Tel el-Daba' on the Pelusiac branch of the Nile developed into an international port of call and apparently controlled much of the trade with Egypt from this time until its destruction at the end of MBIIC. Analysis of settlement patterns ${ }^{75}$, pottery distribution $^{76}$ and Neutron Activation Analysis of some of the large number of Canaanite vessels found at the site indicate a concentration of trade relations with the so-called Gaza group of sites located along the southern coast and inland to Lachish and the exclusion of northern sites ${ }^{77}$. This trade in wine, resins and other materials that required ceramic containers appears uni-directional into Tel el-Daba', with no Egyptian pottery moving outward. What was traded in exchange, aside from

\footnotetext{
72. ARNOLD et ait., 1994.

73. REDFORD, 1992.

74. WEINSTEIN, 1975.

75. OREN, 1997a.

76. BIETAK, 1997.

77. MCGOVERN, 2000.
}

small scarabs and trinkets, remains unclear, although the close spacing of the Gaza group sites indicates a population greater than the carrying capacity of the land ${ }^{78}$. Thus in addition to the gold for which Egypt was famous, an abundant array of bulk food stuffs, including fish (as attested from Lachish), of a type that did not require transport in pottery containers, is likely to be involved.

The evidence for the trade in Nilotic fish from this dynamic period, limited as it is, provides some interesting insights, although the lack of chronological precision precludes a detailed examination of the full possible significance. The presence of Nilotic fish along coastal sites such as Tel Kabri, a large and important port with far flung international relations, and Tel Dor is perhaps not inexpected. However, given the general cessation of trade with the north evident from the study of the materials from Tel el-Daba', the recovery of Nilotic fish at the northern sites of Tel-el-Wawayat in Lower Galilee and Sasa in Upper Galilee are surprising. They are the only good contexted evidence for Egyptian goods at these sites. While Tel-el-Wawayat sits astride a trade route that continues to be important in the LBA, it is not a major centre. Sasa is even smaller, but both sites could potentially be on a route to Hazor and therefore the fish bones might illustrate continued relations between Tel el Daba' in the soutl and its counterpart at Hazor, in control of Syrian trade to the north. Their proximity to a large fish-bearing body of water makes one wonder why exotic fish would be imported to such locations, although the importation of Mediterranean fish to Telel-Wawayat (only a distance of $20 \mathrm{~km}$ away) makes it clear that mechanisms were in place to do so. The lack of evidence for exotic fish at more major nodes or termini of such trades routes makes it difficult to determine whether the presence of fish is simply part of an internal interaction between coastal sites and their hinterlands ${ }^{79}$ or part of a directed trade caravan to a specific inland destination. The same is true for Jerusalem, where Nilotic fish constitute the only evidence for Egyptian contact at this time. The presence of Mediterranean fish at Jerusalem, though not necessarily brought there through the same mechanisms, indicates that the site is being serviced, possibly via Lachish in return for materials (possibly resin or incense) from the Jordan valley. Thus it is unlikely that Jerusalem, although known from the Execration texts, was in direct contact with Egypt.

\footnotetext{
78. OREN, 1997b.
}

79. See ILAN, 1995. 
Megiddo, a regional centre within the Jezreel valley with strategic control of important land routes also holds evidence of the movement of exotic fish in Middle Bronze times. A large number of Egyptian artefacts have been found at the site dating to at least the later part of the Middle Bronze Age. The statue of a $12^{\text {th }}$ Dynasty Egyptian official found at the site, although considered by some as evidence of Egyptian administrative or trading presence at the site, is unlikely to have come to the site until later ${ }^{80}$, nevertheless, reciprocal trade relations, however indirect, with Egypt are indicated by the Neutron Activation Analysis of one ceramic vessel at Tel elDaba' in MBAllA ${ }^{81}$ and the number of Egyptian artefacts points to continued interaction during the Hyksos period.

Egyptian military reprisals to eradicate the Hyksos disrupted the integrated MBA urban landscape of the southern Levant. In the Late Bronze Age, the area of Canaan fragmented into a number of squabbling city states, a situation that Egypt, eventually to become the major force in the region, was happy to maintain, as it allowed it to control and exploit the area with a minimum of man-powers2. International trade with the Aegean world flourished, enriching both Egypt and its vassal city states. At the end of the Late Bronze Age, the Egyptian approach to controlling its diminishing empire changed to a more pro-active one in which Egyptian administrators, garrisons and large quantities of Egyptian material culture (as imports or imitations) were present. The LBA is a well-documented period in which many events - military campaigns, political arrangements, population movements are known, all of which certainly had an impact on trade relations ${ }^{83}$. The sample bias and lack of temporal precision for the majority of the finds of exotic fish preclude their interpretation in light of the complex historical record. Finds of Nilotic fish dated to the Late Bronze Age, mainly in present-day Israel, have been reported from settlements along the coast (Haruvit, Tel Dor, Tel Abu-Hawam, Tel Akko) and from inland sites (Neve Yarak, Megiddo, Wawayat). Nilotic fish imports from more precisely dated contexts are known from Tel Harassim (Late Bronze Age Ila) and Lachish (Late Bronze Age III), and further north, in present-day Lebanon, from a Late Bronze Age II context at Sarepta. Three sites, finally, dated to the Late Bronze Age/Iron Age I are known : coastal Tel Ashqelon, and the inland sites Timna and Tall al'Umayri. The increase, although modest, in the documented

80. WEINSTEIN, 1975: 12.

81. MCGOVERN, 2000.

82. BUNIMOVITZ, 1995.

83. See WEINSTEIN, 1981 ; REDFORD, 1992. export of fish in this period begs the question of the status of fish as an item of trade. We know that fish were transported by boat internally within Egypt, collected as taxes, stored in royal and temple storerooms, and distributed as pay and rations to boat crews, at the Harim, and workmen's villages ${ }^{84}$. In Egypt, fish was a cheap food source (although prices varied by type and whether gutted or whole) on a par with bread and beer and appears to have constituted a major source of protein for workers employed by the state ${ }^{85}$. It may be possible to view the Nilotic fish in this light, i.e., as rations, at least at the following sites dating to the later LBA : Timna, a mining site manned by Egyptians; Haruvit, one of the large fortresses and administrative centre controlling the land route across northern Sinai (the Ways of Horus); and Tel Abu-Hawam (if it is an Egyptian naval base, although this is a matter of debate ${ }^{86}$ ). It is also quite tempting to see the isolated late MB/early LBA find from Tell el-Oreme as the residue of early Egyptian military interests in the north, given the royal stele found there mentioning a victory over foreigners from Mitanni ${ }^{87}$. Evidence for a strong Egyptian presence at Megiddo, Lachish and Ashqelon is also present (again although its significance is debated ${ }^{88}$ ) and may gain additional strength in light of the fish remains. Of course, the export of fish as rations/pay for those in Egyptian employ does not preclude trade, as the presence of Nilotic fish on Cyprus and at Sarepta would imply. It remains unknown whether this took place at a state level or on a more private basis (once the necessary taxes were paid), as depicted in the tomb of Kenamon, where Syrian traders are apparently off-loading their wares in a dock-side market in exchange for textiles, sandals and foodstuffs ${ }^{89}$. That the majority of sites reporting exotic fish are also central places (or very close) within their regions, regardless of an Egyptian presence, could also be construed as evidence for trade ${ }^{90}$.

For the Iron Age I period, eight sites yield evidence for the import of Nilotic fish. Five of them are coastal sites (Sarepta, Tell Qasila, Tel Akko, Tel Ashqelon, and Tel Dor), the three others are located inland (City of David, Ein Hagit and Tell Rehov). At least 19 sites with Nilotic fish are known for the Iron Age II, namely the five coastal settlements Tel Ashqelon, Yavneh-Yam, Tel Gerisa, Tell Qasila and Tel Akko, and 14

84. JANSSEN, 1961 ; HELCK, 1961-1970.

85. JANSSEN, $1975: 348-350,481$.

86. See HASEL, 1998 : 100-102 for discussion.

87. WEINSTEIN, 1981.

88. See HASEL, 1998 : 114-116 for discussion.

89. DAVIES and FAULKNER, 1947.

90. BUNinovitz, 1995 : fig 6. 
inland sites: Kuntillet 'Ajrud, Tel Harassim, Tel Mique, Lachish, Tell el-Oreme, Horvat Rosh Zayit, Megiddo, Tell Rehov, Tel Hamid, Tel Kabri, City of David, Ophel, Tell elHesi and Beer Sheva. Evidence for Nile fish is also present at Tel-el-Wawayat, but the context was only broadly dated to the Iron Age. The presence of Nilotic fish bones at eight Iron Age I sites and 19 Iron Age Il sites reveals an increase both in inland sites and in the overall number of sites yielding Nilotic fish.

The following discussion places the export of Nile fish against the broader textual and archaeological background of Egypt's political and commercial relations with Syria-Palestine throughout the Iron Age to early Persian periods (20th $26^{\text {th }}$ Dynasties : ca $1200-525 \mathrm{BC}$ ). It is suggested here that the changing nature, dispersal, and distribution of Nilotic fish in the Levant follows the broad temporal and spatial distributions of Egyptian(izing) artefact percentages in Levantine strata and deposits spanning Iron Age IA-B, Iron Age IIA-C, and the Neo-Babylonian period. These Egyptian(izing) artefacts have been further quantified as percentages within occupation, cultic, and mortuary contexts at 27 Iron Age sites to assess Egypt's material impact and influence upon different aspects of Levantine society ${ }^{91}$. These sites include a Red Sea port, Tell el-Kheleifeh (Gulf of Aqaba), and nine Mediterranean coastal sites : Tell Abu Salima (Sheikh Zuweid), Deir elBalah, Ruqeish, Tell el-'Ajjul, Ashdod, Tell Qasila, Tel Michal, Tyre, and Sarepta. The remaining 17 settlements represent inland sites throughout the coastal plain and hill country of the Negev and Syria-Palestine : Tell Farah South, Beer Sheva, Horvat Qitmit, Kadesh Barnea, Tawilan, Timna, Tell Beit Mirsim, Lachish, Gezer, Tel Mevorakh, Megiddo, Tell Keisan, Tell es-Sa'idiyeh, Beth Shan, Hazor, Alalakh (Tell Atchana), and Carchemish ${ }^{92}$.

Iron Age IA ( $c a 186-1136 \mathrm{BC}$; early $20^{\text {th }}$ Dynasty) is characterized by an approximately 50 -year period of Sea People and refugee raids and migrations throughout the East Mediterranean and the gradual decline of Egypt's northern empire (temp. Sethnakhte and Ramesses III-VI). During this time, Ramesses III and IV dispatched military expeditions against Palestine (Djahy) and Asiatics ${ }^{93}$. State level contact with Syria-Palestine is reflected by the occurrence of various artefacts and monuments of Ramesses III, IV, and VI in this region ${ }^{94}$.

91. MUMFORD, $1998: 3,925-929$.

92. MUNFORD, 1998.

93. WILSON in PRITCHARD, $1969: 260-263$; PEDEN, 1994 : 87, 89, 97 , 141 .

94. See e.g., MUMFORD, $1998: 338-341$; PORTER and MOSs, 1952 : $370,372,374,379,391,404$.
Despite diverse evidence for Egyptian activity in Iron IA Palestine, and turquoise and copper mining expeditions to South Sinai and the southern Arabah (e.g., Timna, site 200), relatively few contemporary texts survive detailing Egyptian activities in Syria-Palestine at this time. For instance, Papyrus Harris I reveals that Ramesses $11 \mathrm{l}$ established captives in military garrisons, presumably including forts in Palestine (e.g., Deir el-Balah ; Tell el-'Ajjul ; Beth Shan), providing clothing and provisions to them annually ${ }^{95}$. This document mentions Ramesses III building a temple to Amun in Canaan and outlines his construction and dispatch of ships to Syria-Palestine to retrieve annual dues from temple estates in nine towns (other tribiite from this region included cedar, oil, grain, livestock, and captives).

Set against this Egyptian state level activity in Iron Age IA, the extant find spots for Nilotic fish have been noted at only eight Iron Age I sites, namely the coastal sites of Sarepta, Tell Qasila, Tel Akko, Tel Ashqelon, and Tel Dor, and the inland sites of Ein Hagit (near the coast), Tell Rehov, and the City of David (Jerusalem). In contrast, the Iron Age IA strata and deposits at Tell Abu Salima, Deir el-Balah, Tell Beit Mirsim, Ashdod, Tyre, Lachish, Tell Mevorakh, Megiddo, Beth Shan, Deir Alla, Tell es-Sa'idiyeh, Hazor, Sarepta, and Alalakh have yielded a broad range of Egyptian(izing) artefacts amounting to $17 \%, 26 \%$, and $35 \%$ in occupation, cultic, and mortuary contexts, respectively - excluding pottery, which is less well-quantified in many site publications ${ }^{96}$. Of these sites, only Sarepta has yielded Nilotic fish (from an Iron Age I context). Egypt's overall effect upon the Iron IA artefact assemblage amounts to $23 \%$ (149 of 649 artefacts ; excluding Timna's LB IIB-Iron IA shrine : 396 Egyptian items [27\%] out of 1482 artefacts). The Egyptian items consist of figurines (bronze deities; cobras; shawabti funerary figurines), ceramic anthropoid coffins, jewellery (beads; amulets; scarabs and cylinder seals with Egyptian motifs), pottery (bowls, some bearing hieratic texts ; duck-headed bowls ; jugs ; beer jars ; store jars), vessels of calcite and faience, cosmetic containers of calcite and ivory (e.g., fish dishes and kohl-pots), bronze wine sets, gold foil, ivory inlay (from boxes), senet game boards and playing pieces, ostraca (with hieratic texts), bronze tools (e.g., an adze), bronze strips from a city gate, and architecture and monuments ( $\mathrm{T}$-shaped door sills, jambs,

95. IVILSON in PRITCHARD, $1969: 262$.

96. MUMFORD, $1998: 3,928-929$. 
lintels, cornices, capitals, stelae, statues, and statuettes from temples, residences, and forts) ${ }^{97}$.

The export of Nilotic fish in Iron Age IB ( ca 1 136-1 000 $\mathrm{BC}$; late $20^{\text {th }}$ Dynasty - early $21^{\text {st }}$ Dynasty) is mostly undistinguished from Iron IA, but the published find spots have a more limited distribution than Egyptian(izing) artefacts in Iron Age IA Palestine. Concerning Egyptian royal activity, although Iron Age IB lacks firm evidence for Egyptian military activity in Syria-Palestine, it is possible, albeit speculative, that Psusennes I's epithet "seizer of cities" reflects military activity in Palestine ${ }^{98}$. Official Egyptian contact with southern Palestine is implied by the presence of the names of Ramesses VIII, IX, and X on scarabs from Tell Farah South, Tell Masos, Gezer, and Beth Shemesh ${ }^{99}$.

The Journey of Wenamon, a text that is set at the end of Ramesses XI's reign ( $c a 1070 \mathrm{BC}$ ), provides clear information relating to the export of Nilotic fish to Syria-Palestine. This account reveals regular, late Ramesside maritime commerce, albeit through Levantine shipping, between Tanis (northeast Delta), Dor, and Byblos ${ }^{100}$. It relates the transportation to Byblos of Wenamon (a priestly envoy of Amun), a cult statuette of Amun (in Wenamon's possession), five jars of gold, a Kak-men of gold, nine jars of silver, a sack of silver, ten pieces of royal linen, 15 kherd of good Upper Egyptian linen, 500 pieces of unfinished cowhides, 500 ropes, 500 rolls of finished papyrus, 21 sacks of lentils, and 35 baskets of fish $^{101}$. Most of these products represent the payment - to the prince of Byblos - for cedar logs. Five kherd of linen, one sack of lentils, and five baskets of fish are intended for Wenamon's personal use. This document also shows Egyptian traders, messengers, and others (e.g., an Egyptian singer and butler) traveling to and sometimes settling in Byblos. An Assyrian text from year three of King Assur-bel-kala ( $c a 1070 \mathrm{BC}$ ), records the receipt of a royal gift of an ape and a crocodile from Egypt ${ }^{102}$, illuminating the continuation of Egyptian, long distance diplomatic relations after the loss of Egypt's northern empire.

97. Ibid.: I, 597-603, 2, 95-100, 2, 391-399, 3, 344-349, 3, 492-499, 3, $572-578,3,664-672$, and $3,825-832$.

98. REDFORD, $1992: 313 ;$ KITCHEN, $1986: 267$

99. PORTER and MOSS, $1952: 370,374$ and 375 .

100. WILSON in PRITCHARD, $1969: 25-29$.

101. lbid.: 26-28.

102. MLLLARD, $1970: 168-169$.
Although it is not yet possible to observe fluctuations in the quantities of Nilotic fish exports during the Iron Age I period, an examination of Iron Age IB artefact assemblages in occupation, cultic, and mortuary contexts (at Ashdod, Tell Qasila, Tyre, Sarepta, Beer Sheva, Tell Beit Mirsim, Gezer, Megiddo, Beth Shan, Hazor, and Tell Keisan) shows a distinct decrease in Egyptian(izing) items from Iron IA levels, falling to $7 \%, 12 \%$, and $19 \%$ in Iron IB, respectively 103 . Of these sites, Tell Qasila and Sarepta have yielded Nilotic fish from Iron Age I contexts, while most to all of the Iron Age IB strata at these sites have produced Egyptian(izing) artefacts. In this period, Egypt's overall material impact upon the small find assemblage decreases to $10 \%$ (100 of 1002 artefacts). There is a corresponding reduction in the diversity of Egyptian items and Egyptian motifs, which include figurines (bronze deities ; locally made ceramic Ashdoda-figurines with lotus motifs), an Egyptianizing naos-plaque, jewellery (beads; amulets ; scarabs and cylinder seals with Egyptian motifs), seal-impressions, pottery (spinning bowls; duck-headed bowls; store jars), calcite containers and lids, cosmetic containers (kohl pots), inlay (from boxes), senet game boards and counters, and Nile molluscs ${ }^{104}$. Iron Age IB strata and deposits in towns, shrines, and tombs also display some re-use of Egyptian monuments and architectural pieces (door sills ; jambs, cornices ; wall blocks ; mortuary stelae ; a statue of Ramesses III).

The recovery, dating, and publication of fish bones from archaeological sites has improved greatly, but much of the extant data concerning Nilotic fish exports lacks a finer chronological attribution than the general Iron Age I period. Iron Age I encompasses a 200 year period that witnessed radical changes in East Mediterranean shipping, overland trade routes, and the socio-political, cultural and economic infrastructure of the Levant. These changes include the loss of Egypt's Mediterranean fleet, Egypt's dependence upon Levantine shipping (reflected in the Journey of Wenamon), the disappearance of Egypt's fortified military and commercial way-stations ("Ways of Horus") across North Sinai, the Sea People and refigee migrations, the displacement of former populations (e.g., Canaan ; Amurru), the formation of Philistine and Phoenician city-states, and the appearance of Israelite settlements in the hill country.

103. MUMFORD, $1998: 3,928.929$

104. Ibill.: 2, 8-11, 2, 95-100, 2, 434-436, 2, 437-441, 2, 511-515, 3, 344$349,3,492-499,3,529-533,3,572-578,3,664-672$ and $3,730-737$. 
A study of Egyptian(izing) products at Levantine, Iron Age IA-B coastal sites reveals an expected, overall southnorth decrease - using regression analysis - in the material culture assemblages from Tell Abu Salima (Northeast Sinai), Deir el-Balah, Ashdod, Tell Qasila, Tell Mevorakh, Tyre, and Sarepta ${ }^{105}$. Of these coastal sites, Tyre retains the highest proportion of Egyptianizing products (strata XIV-XIII.1 : $17 \%$; $18 \% ; 6 \%$ ) in contrast to Tell Abu Salima (strata L2-3: $13 \% ; 5 \%$ ) and the northern port of Sarepta (strata V/F-VI/ E : $0 \% ; 4 \%)^{106}$. The results from Tyre, however, do represent a smaller artefact assemblage. Until more artefacts are quantified from Tyre, the proportions of Egyptian exports and influence show Tyre competing with the ports at Tell Qasila (strata XIIB-X : $0 \% ; 3 \%$; Iron IB shrines XII-X: $17 \%$; $19 \% ; 17 \%$ ) and Ashdod (strata XIIIa-XI : $10 \% ; 11 \%$; $15 \%)^{107}$. The data reveal a late Iron IB, southern shift to Ashdod concerning the concentration of Egyptian products. In regards to Nilotic fish and shell exports to Iron Age I sites, Nile perch is shipped to Tell Qasila, Tel Akko, Sarepta, and Ein Hagit ( $8 \mathrm{~km}$ from the Mediterranean), Nile catfish is exported to Tel Dor (in Iron Age IA), and Spathopsis shells appear in Jerusalem ${ }^{108}$. After Iron Age IA, as related in the Journey of Wenamon ( $c a 1070 \mathrm{BC}$ ), it is likely that Levantine merchants transported Nilotic fish and other Egyptian products by sea, using non-Egyptian ships, stopping at various ports, and possibly favouring certain ports (e.g., Ashdod ; Tell Qasila ; Tel Dor ${ }^{109}$; Tyre ; Byblos) in contrast to other, perhaps less frequently visited ports (e.g., Tell Abu Salima; Sarepta).

The collapse of Egypt's empire in Iron Age IA removes Egyptian inland garrisons from Syria-Palestine (e.g., at Lachish ; Jerusalem ; Beth Shan) and marks a shift in the dispersal patterns and quantities of Egyptian exports and influence at inland sites. A comparison between the Late Bronze Age and Iron Age west-east (inland) dispersals of Egyptian(izing) products and influence reflects the loss of major Egyptian inland centres in Palestine and a return to a more normal, west-east decrease in Egyptian artefact percentages. In addition to various Egyptian products (mostly luxury items), overland caravans delivered Nilotic fish to diverse inland destinations, such as Ein Hagit $(8 \mathrm{~km}$ from the Mediterranean), Tell Rehov (45 km inland), Jerusalem (50 km

105. Ibid. : 3, 928-929.

106. Ibid. : 1, 602-603, 3, 577-578, 3, 734-737.

107. Ibid. : 2, 99-100, 2, 514-515.

108. REESE et al., $1986: 82$.

109. Cited in the Journey of Wenamon. inland), and even as far as Tall al-'Umayri in Jordan. The $45 \mathrm{~km}$ to $50 \mathrm{~km}$ overland, linear distances actually represent longer routes, following winding wadis and sloping terrain ; these routes could be traversed in two to four days, depending upon whether the caravans used donkeys (17-31 km per day) or ox-drawn carts ( $16 \mathrm{~km}$ per day) to transport goods ${ }^{110}$. With the exception of the southern Negev in Iron Age IB, Egyptian products and Nilotic fish are found at a range of large to small ports and inland settlements during Iron Age I. This probably reflects both a Levantine desire for Egyptian fish and other items and the presence of Egyptian diplomatic gifts, envoys, merchants, and residents living in Syria-Palestine. In exchange, Egypt received cedar, olive oil, wine, bitumen, and a variety of other materials and products shipped from SyriaPalestine and further abroad (e.g., lapis lazuli from Afghanistan).

Although the broad dating of Nilotic fish exports to Iron Age II reflects several centuries, a closer examination of the chronological sequence of Egypto-Levantine historical and material cultural relations clarifies the political and economic setting for Nilotic fish exports. Iron Age IIA ( $c a 1000-925$ $\mathrm{BC}$; late $21^{\text {st }}$ Dynasty - early $22^{\text {nd }}$ Dynasty) witnesses a reappearance of some Egyptian military activity in southern Palestine. A temple wall block from Tanis portrays Siamun ( $c a$ 978-959 BC) smiting an Asiatic, while his name occurs on a scarab from Tell Farah South ${ }^{111}$. A later Biblical text ${ }^{112}$ mentions an Egyptian raid ascribed to this time. The best attested military activity takes place at the end of this period, ca 925 $\mathrm{BC}$, during the reign of Sheshonq I ( $c a$ 945-924 BC). His extensive campaign against southem Palestine and the Negev is attested on a stele fragment from Megiddo, several inscriptions in Egypt, and Biblical accounts ${ }^{113}$. Sheshonq I apparently also maintained diplomatic and commercial ties with Byblos, which yielded a votive statue of Sheshonq I $^{114}$. Later Biblical texts mention the presence of Egyptians in Israel during the reign of King David ${ }^{115}$, the marriage of an Egyptian princess to King Solomon ${ }^{116}$, and the export of Egyptian chariots and horses to Israel117.

110. ENGELS, 1978: 15-16, note $15,153-156$, table 7 ; MUMFORD, $1998: 42 \cdot 48$, table 1.10

111. KITCHEN, $1986: 280-281$; PORTER and MOSS, $1952: 370$.

112. I Kings $9: 15 \cdot 18$.

113. 1 Kings $14: 25-27 ; 2$ Chronicles $12: 25-27 ;$ KITCHEN, $1986: 432$ 447 ; PORTER and MOSS, $1952: 381$.

114. PORTER and MOSS, 1952: 388.

115. 2 Samuel $23: 21 ; 1$ Chronicles $11: 23$.

116. I Kings $3: 1-2,9: 15-24$.

117. I Kings $10: 28-29 ; 2$ Chronicles $1: 16-17$. 
Concerning Egyptian contact with Syria-Palestine, however, there is a small to "large" decrease in Egyptian(izing) artefacts from Iron IB to $9 \%, 0 \%$ (small sample), and $17 \%$ in the Iron IIA material culture assemblages of occupation, cultic, and mortuary contexts at Tell Abu Salima, Ashdod, Beer Sheva, Tell Beit Mirsim, Lachish, Tell Qasila, Tel Michal, Tell Mevorakh, Megiddo, Beth Shan, Hazor, Tell Keisan, Tyre, and Sarepta118. Of these sites, Beer Sheva, Lachish, Tell Qasila, and Megiddo have yielded Nilotic fish from Iron Age II contexts, while Tell Qasila, Tel Michal, Tell Mevorakh, Hazor, Tyre, and Sarepta lack Egyptian(izing) artefacts in their Iron Age IIA strata. The overall Egyptian influence in the Iron Age IIA artefact assemblage falls slightly from Iron IB to $9 \%$ (29 of 309 items). There is also a dramatic reduction in the range of Egyptian artefact types, which consist of jewellery (beads; scarabs and scaraboids; plaque seals), pottery (spinning bowls; duck-headed bowls; beer jars), a bronze fish-shaped weight, and Nile molluscs ${ }^{19}$. This picture might be modified by the quantification of more sites and artifact assemblages from Iron Age IIA.

The first half of Iron Age IIB ( $c$ a 924-732 BC; late $22^{\text {nd }}$ Dynasty $-23^{\text {rd }}-24^{\text {th }}$ Dynasties) exhibits a continuation of Egyptian military and related activity in the Levant. Osorkon I ( $c a$ 924-889 BC) may be equated with a person called Zerar, who conducted a raid into Palestine ${ }^{120}$; he is represented by a votive statue at Byblos and a scarab from Salamis (Cyprus) $^{121}$. Around 853 BC, Osorkon II (ca 874-850 BC) probably represents the Egyptian king who sent 1000 troops to assist a Levantine coalition against the Assyrians at the Battle of Qarqar (Syria) ${ }^{122}$; his political ties with the Levant are attested by the presence of his name on a vase fragment from Samaria (Israel) and a votive statue at Byblos ${ }^{123}$. Osorkon IV (ca 730-715 BC) probably maintained diplomatic relations with Philistia and Israel according to Assyrian and later Biblical texts. These texts mention that the king of Egypt ${ }^{124}$ dispatched his commander and troops against the Assyrians in southern Palestine ${ }^{25}$. Towards the end of Iron Age IIB, other Assyrian and Biblical texts describe Egypt's export and transportation to the Levant (and Assyria) of Egyptian horses, sol-

118. MUMFORD, $1998: 3,928-929$.

119. Ibid.: 1, 597-591, 603, 2, 8-11, 2, 95-100, 2, 391-399, 2, 434-436, 2 , 471-474, 3, 492-499, and 3, 529-533.

120. 2 Chronicles $14: 9-15$.

121. PORTER and MOSS, $1952: 388$; LECLANT, $1967: 218$ no. $6 \mathrm{~b}$.

122. OPPENHEIM in PRITCHARD, $1969: 278-279$.

123. PORTER and MOSS, $1952: 376,388$.

124. Named "So" in 2 Kings $17: 1 \cdot 6$.

125. OPPENHEIM in PRITCHARD, $1969: 284-286$. diers (Qarqar), camels, a hippopotamus, a rhinoceros, an antelope, elephants, and monkeys ${ }^{126}$.

Iron Age IIB may represent a period of increased exportation of Nilotic fish to Palestine. For instance, at Tell Abu Salima, Tell el-'Ajjul, Ashdod, Tell Qasila, Tel Michal, Tyre, Sarepta, Tell Beit Mirsim, Lachish, Gezer, Megiddo, Tell esSa'idiyeh, Hazor, and Carchemish Egyptian(izing) artefacts increase variously from Iron IIA to $9 \%, 44 \%$ (small sample), and $15 \%$ in Iron IIB occupation, cultic, and mortuary artefact assemblages, respectively ${ }^{127}$. Of note, Tell Qasila, Lachish, and Megiddo have yielded the remains of Nilotic fish from Iron Age II contexts, while Tell Beit Mirsim and Tel Michal lack Egyptian artefacts from their Iron Age IIB strata. It must be emphasized, however, that in the overall archaeological data quantified for this period, Egyptian artefacts rise only a little to $11 \%$ (284 of 2695 artefacts). A more detailed examination of five sub-phases of Iron Age IIB has revealed a gradual decline in Egyptian influence (i.e., artefact percentages) in Palestine in relation to increasing Assyrian control ${ }^{128}$.

The Egyptian and Egypto-Phoenician artefact types include figurines (a monkey; a shawabti funerary figurine from a shrine), jewellery (beads; amulets; scarabs, scaraboids, plaque seals, and cylinder seals with Egyptian motifs), pottery (duck-headed bowls; beer jars ; store jars), calcite, ivory, and clay cosmetic containers (kohl pots; an alabastron; a pyxis), vessels of calcite and faience, senet game boards and counters, a 20 -square game board, linel from tombs (near Carchemish), Egyptian weights (with hieratic numbers), and fittings (e.g., an Egypto-Phoenician decorated bone handle) ${ }^{129}$. In southern Syria and Assyria, Phoenician ivory inlays from furniture display many Egyptian motifs ${ }^{130}$.

During Iron Age IIC ( $c$ a 732-605 BC), contact between Egypt and Southwest Asia intensifies dramatically ${ }^{131}$. At this time, although Kushite Egypt had managed to dispatch troops - led by Prince Taharqa - against Assyria in $701 \mathrm{BC}$, the Assyrians kept Egypt on the defensive, attacking and sometimes briefly occupying Egypt during ca 674, 671, 667, and $664 \mathrm{BC}$. The continuous, albeit changing, Kushite relations with Palestine and Assyria are also illustrated by the car-

126. Ibid. : 278-279, 281 ; KITCHEN, $1986: 324$

127. MUAFORD, $1998: 3,928-929$.

128. Ibid. : $3,928-929$.

129. bid. : 1, 748-750, 1, 949-961, 2, 95-100, 2, 391-399, 2, 434-436, 2 , $437-441,2,471-474,2,511-515,2,796-802,3,344-349,3,572-578,3,664$ $672,3,730-737$, and $3,910-912$.

130. BARNETT, 1982.

131. STERN, $2001: 228-235$. 
touches of Shabako ( $\mathrm{ca}$ 716-702 BC) on scarabs from Tell Farah South and Beth Shan, jar sealings from Megiddo, correspondence sealings from Nineveh (Assyria), and a statue from Palestine ; likewise, the name of Taharqa ( $c a$ 690-664 $\mathrm{BC}$ ) occurs on a sealing from Tadmor (Palmyra), a vase from Ashur (Assyria), and statuettes (i.e., booty) from Nineveh ${ }^{132}$.

The advent of the $26^{\text {th }}$ Dynasty (Saite period) marks the decline of the Assyrian empire and a brief period of Egyptian imperialism. For instance, Kings Psamtik I and Necho II dispatched troops to Syria-Palestine to assist the weakening Assyrian empire in $616,612,610-609$, and 606-605 BC. This phase of Saite imperialism in the Levant is also reflected through the name of Psamtik I ( $c a$ 664-610 BC) on scarabs from Gezer, Atlit, and Salamis, and statues from Taanach and Arvad (Phoenicia); similarly, the cartouches of Necho II ( $\mathrm{ca}$ 610-595 BC) are found on a statue from Sidon and jar sealings from Carchemish (Egyptian and Assyrian military headquarters) ${ }^{133}$.

The recovery and quantification of Nilotic fish from specific Iron Age IIC contexts would augment our understanding of Egypto-Levantine relations during this particularly turbulent period in international relations. For instance, although late Iron Age texts provide selective details regarding Egypt's relations with Syria-Palestine and Assyria, they lack references to the export of Nilotic fish to the Levant. Instead, texts identify Egyptian items of trade, tribute, and booty as including grain, horses, rams, donkeys, monkeys, leather products, cow hides, obelisks, unspecified items (of wood, gold, silver, copper, tin, stone, and ivory), precious stones, jewellery, crowns, alum (?), linen (including clothes and sail cloth), byssos-quality linen, pieces of African blackwood ("ebony"), furniture, unspecified items, and the personal possessions belonging to King Tanwetamani ${ }^{134}$. In addition, the Egyptian and Kushite captives transported back to Assyria consisted of Taharqa's queen, the crown prince, female servants, courtiers, physicians, divination experts, goldsmiths, cabinet-makers, and others ${ }^{135}$.

Despite an initial expectation that Iron Age IIC would reveal an increase in Egyptian products, the quantification of artefact assemblages at Tell Abu Salima, Horvat Qitmit, Beer Sheva, Tawilan, Tell el-Kheleifeh, Tell Beit Mirsim, Lachish, Gezer, Ashdod, Tell Qasila, Tell es-Sa'idiyeh, Megiddo, Tell

132. PORTER and MOSS, $1952: 370,379,381,382,396$, and 397 ; LECLANT, $1958: 96$ no. $6 \mathrm{~b}$; LECLANT, $1961: 394$ no. $7 \mathrm{a}, 395$ no. $7 \mathrm{~b}$.

133. PORTER and MOSS, $1952: 375,380,381,384,393,398$; LECLANT, $1961: 395$ no. 8 ; LECLANT, $1969: 296$ no. 3c.

134. OPPENHEIM in PRITCHARD, $1969: 293-295,297,302-303,392-393$.

135. Ibid. : 293.
Keisan, Hazor, Sarepta, and Carchemish, demonstrates that Egyptian(izing) artefacts actually decrease somewhat from Iron IIB, falling to $5 \%, 2 \%$, and $13 \%$ in Iron IIC occupation, cultic, and mortuary contexts, respectively ${ }^{136}$. Of these sites, Beer Sheva, Lachish, Tell Qasila, and Megiddo have yielded Nilotic fish from Iron Age II contexts, while Tell Qasila, Tell es-Sa'idiyeh, Megiddo, and Hazor lack Egyptian(izing) artefacts in their Iron Age IIC strata. Egyptian influence within the overall Iron Age IIC artefact assemblage drops to an average of $4 \%$ ( 89 of 2277 artefacts).

The Egyptian(izing) artefacts included figurines (a baboon ; bronze statuettes of Osiris, Isis, and Horus), jewellery (beads ; amulets ; scarabs), a signet ring of Psamtik I, seal impressions (e.g., Necho II), pottery (store jars), vessels of faience, calcite, and granite, cosmetic containers (e.g., ivory kohl pot), Nile shells (Spathopsis from Ashdod stratum V), bronze wine sets, bronze situlae, ivory carvings (from furniture), a stone sistrum (Tel Miqne-Ekron), a clay mould for a Bes amulet (Gezer), and ostraca bearing hieratic numbers and measures (e.g., Kadesh-Barnea ; Arad) ${ }^{137}$. Although Phoenician architecture adopted various Egyptian architectural elements during Iron Age II, the late $7^{\text {th }}$ century $\mathrm{BC}$ contains clear evidence for an Egyptian structure in Syria : Carchemish has yielded an Egyptian-style official residence with $\mathrm{T}$ shaped door sills, five uninscribed wall slabs topped by a cavetto cornice, and many Egyptian items ${ }^{138}$. Tyre has produced a diorite head from the statue of a $25^{\text {th }}$ Dynasty king 139 .

Iron Age IIA-C spans a 400-year period that is characterized by the rise of Phoenician city-states, Phoenician maritime commerce, and fluctuations in the socio-economic and political relations between Egypt, Philistia, Israel, Judah, Edom, Moab, Ammon, Aram-Damascus, Phoenicia, and Assyria. In general, after $c a 853 \mathrm{BC}$, the rise and westward expansion of the Assyrian empire appears to have reduced Egypt's material influence and trade in the Levant.

The distribution of Egyptian(izing) artefacts at Levantine Iron Age II coastal sites displays a continuation of the Iron Age I pattern of a south-nortl decrease (confirmed through regression analysis) in Egyptian influence in various subphases of Iron Age IIB artefact assemblages. This pattern is likely duplicated by Nilotic fish exports and relies mainly on

136. MUMFORD, $1998: 3,928-929$.

137. Ibid. : 1, 416-429, 1, 597-603, 2, 8-11, 2, 95-100, 2, 391-399, 2, 434. $436,2,437-441,3,529-533,3,572-578$, and 3, 910-912; STERN, $2001: 228$ 235.

138. WOOLLEY, $1921: 123-128$.

139. PORTER and MOSS, $1952: 383$ 
occupation contexts at Tell Abu Salima (strata K1-Lower G : $40 \% ; 33 \% ; 41 \% ; 29 \% ; 37 \% ; 23 \% ; 8 \% ; 18 \%$ ), Ashdod (strata X-VI : $11 \% ; 6 \% ; 13 \% ; 4 \% ; 4 \%$ ), Tell Qasila (strata IX-VII : $0 \% ; 7 \%$ ), Tel Michal (strata XIV-XIII : $0 \% ; 17 \% ; 900-730 \mathrm{BC}$ hiatus ; stratum XII : $0 \% ; 700-540$ $\mathrm{BC}$ hiatus), Tell Mevorakh (Iron IIA strata VIII-VIl : $0 \%$ ), Tyre (strata XII-X : $0 \%$; IX-VIII : $9 \%, 25 \%$; VII-V : $0 \%$; IV-III : $18 \%, 17 \%$; II-I : $0 \%$ ), and Sarepta (strata VII/D-B : $0 \%$; Iron IIC-Persian: $4 \% ; 800-600$ BC Shrine-1: $34 \%)^{140}$. In the second half of Iron Age IIB, ca $850-720 \mathrm{BC}$, peaks occur in Egyptian trade and influence at the Phoenician port of Tyre (strata IX-VIII : $9 \%, 25 \%$; IV-III : $18 \%, 17 \%$ ) in contrast to lower levels of Egyptian(izing) items at Ashdod (strata IX-VII : $6 \% ; 13 \% ; 4 \%$ ). These northern peaks are short-lived, however, and, despite a brief decade of Saite imperialism ( $c a$ 616-605 BC), Egyptian influence decreases in northem Palestine throughout Iron IIC.

In Iron Age IIA-C, Nilotic fish and Nile shells (Spathopsis from Tell Goren and Jerusalem) are exported by ships to various ports (cited above) and re-dispersed inland more broadly by overland caravans to markets in Philistia (Tel Ashqelon; Tel Miqne-Ekron), Edom/Negev (Beer Sheva; Tell Rehov; Horvat Temam), Judah (Tell el-Hesi ; Lachish; Jerusalem [Ophel and the City of David] ; Tell Goren), Israel (YavnehYam; Tel Gerisa; Tell Qasila ; Tel Hamid ; Megiddo ; Beth Shan; Hazor; Tell el-Oreme; Tell es-Sa'idiyeh), Moab (Tawilan), Ammon (Tall al-'Umayri), the Kingdom of AramDamascus, and Phoenicia (Tel Akko; Tell Keisan; Tel Kabri ; Tel Harassim) ${ }^{141}$.

At the advent of Iron Age II, the formation of the United Monarchy in Israel and its subsequent expansion into the Negev secured passage to the Gulf of Aqaba and the Red Sea spice and incense trade. Sheshonq I's military campaign into the Negev probably aimed to secure or gain revenue from this lucrative, Red Sea tradel42. Later, during the Iron IIB-C periods, Egyptian(izing) artefacts, and in many cases Nilotic fish, appear at sites extending from the coastal ports of Ruqueish (an Assyrian trading centre : Sargon II's sealed Karmm) and Tell el-'Ajjul, past Tell Farah South, Tell Rehov, Kadesh Barnea, and Horvat Teman, to Tell el-Kheleifeh beside the Gulf of Aqaba ${ }^{143}$. Another caravan route branches off eastwards from Tell el-'Ajjul to Beer Sheva, Horvat Qitmit, and Tawilan.

140. MUMFORD, $1998: 1,602-603,2,9-10,2,473-474,2,514-515,2$, $944-945,3,577-578,3,734-737$.

141. REESE et al., $1986: 82$.

142. MUMFORD, $1998: 386-388,1,439-440$.

143. Ibid. : 925-926.
Various rivers and valleys mark other caravan trails leading inland from the Mediterranean Sea to the Plain of Sharon (Tell el-Hesi ; Lachish ; Tel Miqne ; Gezer ; Tel Hamid), the hill country (Tell Beit Mirsim ; Jerusalem), the Jordan Valley, and beyond (Tell Hesban; Tall al-'Umayri). Another major overland caravan route heads eastwards through the Jezreel Valley, from Tel Akko to Tell Keisan, Horvat Rosh Zayit, Tel-el-Wawayat, Megiddo, and Beth Shan, while the sites of Tell el-Oreme, Hazor, and Tel Harassim could be reached from northern ports or via a north-south caravan route along the Jordan Valley.

As in the Iron Age I period, Egyptian items and Nilotic fish are found at a range of large to smaller ports and inland settlements during Iron Age II. Egyptian fish, luxury products, and other items appear at a greater number of sites, albeit in lower proportions in local artefact assemblages, possibly reflecting the spread of Egyptian(izing) items from the elite to a larger portion of the population. The Egyptian fish and other products also reflect the presence of Egyptian gifts, messengers, merchants, and residents in this region.

The Neo-Babylonian ( $c a 605-540$ BC) to early Persian period ( $c$ a 539-525 BC) spans the end of the $26^{\text {th }}$ Dynasty in Egypt. This period begins with the 605 BC Babylonian defeat of Egypt and Assyria at Carchemish, which is followed by Egypt losing its domination of Palestine and having to defend its eastern frontier against a Babylonian attack in $601 \mathrm{BC}$. The subsequent Egyptian campaigns into Palestine failed to dislodge the Babylonians. In $591 \mathrm{BC}$, Egypto-Babylonian relations appear to have cooled sufficiently for Psamtik II to send a priestly delegation into Palestine. This calm was short-lived, however, since by $589 \mathrm{BC}$ the Babylonians had to send troops to southern Palestine to suppress a rebellion. An army sent into Philistia and Judah by Apries failed to end the Neo-Babylonian siege of Jerusalem (ca 589 BC) and had limited success attacking Neo-Babylonian forces at Tyre and Sidon ( $\mathrm{ca}$ $586)^{144}$. By $568 \mathrm{BC}$, the Neo-Babylonians resumed their attack upon Egypt's eastem frontier. Towards the end of Iron Age IIB, the name of Amasis (Ahmose II) occurs on a sistrum from Sidon and Psamtik III's name appears on a scarab from 'Ain Shems' 45 . By 539 BC, the Persians had taken over the Babylonian empire, and, in $525 \mathrm{BC}$, succeeded in invading and establishing a long-term occupation of Egypt.

Inscriptions reveal that Egyptian personnel, exports, and booty to southwest Asia included ivory, alum, natron, linen

144. Herodotus Book II : 160-166.

145. PORTER and MOSS, $1952: 373,384$. 
(including fine linen), pieces of African blackwood ("ebony"), unspecified items, soldiers, priests, residents (e.g., Kushite servants), and captives ${ }^{146}$. These texts lack references to Nilotic fish exports.

During this period, Egyptian and Egypto-Phoenician artefacts continue to appear in diverse contexts at Tell Abu Salima (stratum Upper G : $30 \% ; 3$ of 10 items), Beer Sheva (pit 866 votives : $67 \% ; 2$ of 3 items), Tawilan (stages $1-5: 0.4 \% ; 2$ of 496 items), Ashdod (stratum Vc-b: $0 \% ; 0$ of 19 items), Lachish (tombs : $17 \% ; 1$ of 6 items), Tell Keisan (stratum 3 b. $1-3: 17 \% ; 1$ of 6 items), and Sarepta (stratum B : $0 \% ; 0$ of 15 items); the Neo-Babylonian strata and deposits at Ashdod, Lachish, and Sarepta, lack Egyptian(izing) artefacts. These items amount to $1.1 \%$ (6 of 546 items) in occupation contexts, " $67 \%$ " (unreliable sample size : 2 of 3 items) in cultic contexts, and $17 \%$ (unreliable sample size : 1 of 6 items) in mortuary contexts ${ }^{147}$. The combined totals, namely nine Egyptian(izing) artefacts out of 555 items, represent an overall Egyptian impact of $2 \%$, but require further sites, contexts, and artefacts to assess the validity of this result for the Neo-Babylonian period. For example, the exclusion of the artefact assemblage from Tawilan raises the overall, quantified Egyptian influence at the sampled sites from $2 \%$ to $14 \%$.

These and other Egyptian and Egypto-Phoenician artefacts include figurines (a baboon), jewellery (beads; amulets ; scaraboids), vessels of calcite and faience, an ivory cosmetic container, and a bronze mirror ${ }^{148}$. Other Saite period scarabs, vessels, and statuettes appear at Beer Sheva, Tell Zakariya, Tel Miqne-Ekron, Atlit, Deir Sras (east of Lake Hulah), 'Adlun (Phoenicia), Tell Arqa (Phoenicia), Carchemish (Syria), Tell Abu Danne (Assyria), Nineveh, and Persepolis (Iran) ${ }^{149}$; Beer Sheva, Lachish, and Tel Miqne also produced Nilotic fish remains in Iron Age II.

In summation, the detection of Nilotic fish and Egyptian(izing) artefacts at Iron Age sites have clarified Egypt's conmercial relations with the East Mediterranean and Levant. The distribution of these Nilotic fish bones, and the account of Nilotic fish exports to Byblos in the Journey of

146. Ezekiel 27:2-9, 15; FREEDY and REDFORD, 1970:479; ODED, $1979: 57,100,101,104$; OPPENHEIM, $1967: 238,243,245,248,250,251$, $253 ; 1$ Chronicles $2: 34-35$.

147. MUMFORD, $1998: 1,429-433,1,602.603,2,10-11,2,99-100,2$, $396-399,3,532-533,3,736-737$, and $3,928-929$.

148. Ibid. : $1,416-437,3,529-533$, and $3,664-672$.

149. LECLANT, $1961: 395$ no. 7b ; LECLANT and CLERC, $1985: 489$ no. 4d ; LECLANT and CLERC, $1987: 381$ no. 5 ; LECLANT and CLERC, $1989: 378$ no. 3b; LECLANT and CLERC, 1991 : 268 no. $1 \mathrm{~g}$; PORTER and MOSS, 1952 : $372,381,383,398$.
Wenamon, demonstrate that both Egyptian residents abroad and at least the upper echelons of the Levantine populace consumed Nilotic fish. However, the appearance of Nilotic fish at coastal and inland settlements of all sizes supports an argument for a broader spectrum of the Iron Age population consuming imported fish from Egypt. The general Iron I-II increase in sites yielding Nilotic fish is paralleled loosely by various peaks in Egyptian(izing) artefacts at Iron Age II sites. Comparisons between both data sets, however, await the attribution of Nilotic fish remains to narrower time frames within the Iron Age.

Until better chronological sequences and quantifications become available for the excavated and published Nilotic fish remains from Iron Age sites, it remains hypothetical that Iron Age I and II Nilotic fish exports parallel the temporal and spatial dispersal pattems for other Egyptian products in the Levant. An examination of 27 Levantine sites and 19 phases, spanning LB Age IA to the Neo-Babylonian period, has revealed that Egyptian(izing) artefacts and infuence reach a peak in late LB Age IIB ( $c a \mid 250-1200 \mathrm{BC}: 26 \%$ ), late Iron Age IB ( $c a$ $1050-1000 \mathrm{BC}: 11 \%$ ), and early Iron Age IIB (ca 900-850 BC : $14 \%$ ), with an overall decline in Egyptian exports and types of products throughout most of Iron Age II ${ }^{150}$. Of these selected sites, $89 \%$ (of late LB II sites), $79 \%$ (of Iron IA sites), $100 \%$ (of Iron IB sites), $57 \%$ (of Iron IIA sites), $79 \%$ (of Iron IIB sites), $75 \%$ (of Iron IIC sites), and $70 \%$ (of Neo-Babylonian sites) yield Egyptian(izing) artefacts, duplicating the peaks in Egyptian artefacts and influence during the later Late Bronze IIB to Neo-Babylonian periods. Hence, late LB IIB, (late) Iron IB, and (early) Iron IIB yield peaks in both the distribution and quantities (\%) of Egyptian artefacts and influence at Levantine sites. Both the Sea People's raids and the Battle of Qarqar between the Assyrians and Levantine-Egyptian coalition ( $c a$ $853 \mathrm{BC}$ ) signal the advent of major reversals in Egypt's political and commercial influence in Syria-Palestine. The latter conflict marks the advent of Assyria's growing domination of the southern Levant, which, despite a brief period of Saite imperialism, is succeeded by the Neo-Babylonian and Persian control of this region.

The Achaemenid empire expanded in most regions of the Near East, between the accession of Cyrus and the conquest of Alexander. The Persian period is recently the focus of renewed archaeology attention, approaching the concept and praxis of its empire ${ }^{151}$. In the Levant, the Phoenician towns

150. MUMFORD, $1998: 3,928.929$.

151. DUSINBERRE, 2003. 
acted as strong partners, while Cyrus allowed the exiled Jews to return and found a more or less autonomous temple state centred on the temple in Jerusalem ${ }^{152}$. Egypt was brought under Persian control until the end of the $5^{\text {th }}$ century $\mathrm{BC}$, while two local dynasties regained control for most of the fourth century $\mathrm{BC}$. The attested export of Nile fish at Tel Akko, Tel Dor, Tel Harassim and Tel Ashq̨elon does not allow the reconstruction of an intelligible pattern of exchange or the detection of an incentive provided by the incorporation of both regions in the newly established empire. The remains rather suggest a continuity of contact.

Egypt, especially after the foundation of Alexandria, witnessed the opening of new worlds. The new royal Ptolemaic dynasty capitalized on the political and economic potential of the many gifts of the Nile. The fact that Egypt was later turned into a personal protectorate of the Roman emperor further illustrates its potential impact in the contemporary constellation of the eastern Mediterranean. From the Egyptian hinterland a large variety of agricultural products came to Alexandria, but the lands along the Nile were mainly known as granaries. A large part of the Egyptian grain was exported, provisioning Rome and later Constantinople. Egypt, and especially Alexandria, was known for its textiles and the preparation of eastern silk and perfumes. Only the marshy Nile Delta offered the perfect ecological conditions for the papyrus plant, the main writing material in ancient times. No doubt the many medicines prepared by the famous medical school at Alexandria were also in high demand. In the Eastern Desert and along the Nile in Upper and Middle Egypt were many stone quarries where especially limestone, sandstone, basalt, granite and porphyry could be mined. These quarries were run by the state. Egypt and mainly Alexandria was famous for the quality of its craftsmanship, although there is very little archaeological evidence available to attest and identify Alexandrian production. High quality Alexandrian glass vessels were exported, and Alexandrian gold, silver and bronze vessels were also in great demand, as well as the products of the local jewellers ${ }^{153}$. Various ancient sources indicate that fish products may also have been a staple export from Egypt. The fish factory at Mendes, for one, was known throughout the Roman empire, and documentary sources further attest to the export of catfish to Rome. Sophokles ${ }^{154}$ discussed the shipment of Egyptian fish to Greece in the $5^{\text {th }}$ century $\mathrm{BC}$,

152. BEDFORD, 2001.

153. EMPEREUR (éd.), 1998.

154. Ap. Athen. $3.119 \mathrm{c}$.
Xenokrates of Aphrodisias told of the fame of Egyptian fish products in the early empire, and Synesitus of Cyrene could not do without dried Egyptian fish at his estate in southern Cyrenaica in late Roman times ${ }^{155}$. The present-day archacological record provides the following framework for the fish trade in the Levant and the Aegean.

For the Hellenistic period only the coastal sites of Tel Akko, Jaffa, Apolonia and Yavneh-Yam yield archaeozoological evidence for trade in Nilotic fish, but papyrology provides futher data. In this context, mainly the Zenon archive is of importance. The archive was a lucky and exceptional find of a coherent collection of documents by local farmers at ancient Philadelphia in the Fayum. The texts represent about one third of the total amount of extant Ptolemaic papyri and are closely datable to between 261 and $229 \mathrm{BC}$. Zenon was born at Carian Katnos when this region was still under Ptolemaic control and soon came to enjoy the benefits of the Hellenistic commonwealtly when he became an aid to Apollonius, a treasury official at Alexandria ${ }^{156}$. The first part of the archive, when Zenon travelled in Palestine, is of main interest here, as it provides detailed information on the wide variety of products purchased by Zenon on behalf of his overlord, illustrating the intricacies and complexities of daily life and contemporary trade activities. Many of the products traded by Zenon, such as Sicilian and Black Sea fish, will have found other, possibly more direct, ways into or from Egypt, while other items of exchange will never have been the concern of Zenon. Even if the archive is very particularistic and difficult to extrapolate, the collection of documents provides a very vivid illustration of the potential deficiencies of the archaeological record and its interpretation. For example it attests to the transaction of 32 drachmes for two camels of dried fish transported from Pelusium to Gaza for which archacological evidence would be difficult to obtain.

Exchange patterns between neighbouring Palestine and Egypt were of course varied and longstanding, either by sea or land. Next to a growing body of papyrological material from the Roman Near East ${ }^{157}$, Talmudic source material also provides unique and highly detailed evidence of patterns of daily life in the towns and countryside of the Land of Israel, comparable in standard to papyri. It is clear that imports from Roman Egypt into contemporary Palestine were varied and represented the largest portion of imported materials 155 .

155. CURTIS, $1991: 131-141$

156. PESTMAN (ed.), 1981.

157. COTTON et al., 1995.

158. SPERBER, 1976. 
Trade between both regions was supported by the fact that the Jews of Alexandria had an important stake in the shipping industry. Imports fiom Egypt included commodities such as grain, linen, for which Pelusium was especially known but which would experience strong local competition in later Roman times, natron for washing clothes, drugs and medicaments from Alexandria, and plaited basketry and ropes.

Food items, in particular, were subject to specific conditions of Jewish religious law, so that, for instance, Egyptian wine, which is reported as an import in Rabbinic sources, was not fit for Jewish consumption, except for the sour varieties. Beer, on the other hand, was mostly tolerated and imported from Egypt. Similar restrictions applied to fisl products. As in other regions of the eastern Mediterranean, fish formed an important element in the diet of ancient Palestine, and most fish were caught in the Mediterranean or the Sea of Galilee. The latter area supplied the Jewish part of the population for the most part, and Jews were also not supposed to buy their fish products from non-Jews. The non-Jewish elements of the population, on the other hand, were mainly concentrated in the coastal towns, where fishermen were a normal feature. Ptolemais, for instance, was an important fishing centre. Ancient testimonies further attest to the importance of fish imports into Palestine, of which Jews considered a few species clean and thus also consumable. Dried Egyptian fish transported in baskets was one of these exceptions and was well known throughout Roman Palestine. Garum from Pelusium, and Apameia and Sidon in Syria, imported into Caesarea, seems to have dominated the late Roman market, and was considered kosher in the Talmudic tradition, in contrast to the shellfish from the Delta ${ }^{159}$. Dried Nilotic fish (except for the scaleless catfish) could thus be consumed by both Jews and non-Jews alike, and considering the close exchange patterns between both Egypt and Palestine, it should not come as a surprise to find imported fish remains throughout Palestine at most of the major excavated sites.

Dried fish was probably imported from Alexandria or Pelusium to the main harbours such as Ptolemais, Tel Dor, Caesarea Maritima, Apolonia, Ashqelon or Gaza. The specific geography of the Palestine coastline limited the position of important harbours to a few locations ${ }^{160}$, where the population would be mixed and engaged in wider exchange patterns, as attested from the pottery spectra, for instance ${ }^{161}$.

159. SPERBER, 1976:119-123; SAFrAI, $1994: 163-165,385-386$

160. SPERBER, $1998: 171-176$.

161. BERLIN, 1992.
Another possibility was, of course, land transport via the Pelusium-Gaza desert route, which connected to the trade artery of the Via Maris or branched inland. The development of the inland road system in Palestine was mainly a Roman incentive, and facilitated traffic between all major towns of the region; however, no apparent traffic nodes or main routes were created ${ }^{162}$. As a result, apart from the main lines of communication, many more or less direct, occasional and smaller scale chamels of distribution will also have been active, sustaining daily exchange, which are mostly beyond grasp in archaeology. The Talmudic tradition, for instance, attests to traffic between the Judaean sea harbours and also along the Sea of Galilee ${ }^{163}$. This more modest type of exchange probably formed the reality for the majority of the population, allowing for many variants of down-the-line trading which are now impossible to pin-point.

Interestingly, the same patterns of wide distribution of Nile fish throughout the Land of Israel seem to continue into the Islamic and Crusader periods, indicating the continuing potential for exchange of needs and benefits between two neighbouring regions.

As far as the Nabataean kingdom is concerned, only Petra yielded Clarias gariepimus, which is said to be possibly local in the original publication. Judging from the modern distribution, the nearest source for this fish within the Levant seems to be the Jordan basin, which is about $170 \mathrm{~km}$ from Petra. Given the intense involvement of the Nabataeans, and the town of Petra in particular, in the incense trade between southern Arabia and the Mediterranean, it is atso possible that this fish may have been imported from the Nile, as part of the aromatics may have been destined for Egypt, and the perfume industry of Alexandria. Well-maintained caravan routes crossed the Negev and Sinai deserts, reaching Gaza or Egypt itself. In this way, Nile fish could have formed part of the retum cargo of these caravans, in much the same way as Nile fish reached the Red Sea harbours. The Zenon papyri illustrate the importance of this exchange under Nabataean control, with incense brought by caravan to the port of Gaza, and entering the Egyptian tax system at Pelusitum. Even caravans that were not destined for Egypt, but the Mediterranean in general, could have stocked Nile fish at the overlay point of Gaza. The desert caravan routes were maintained at least into the Severan period ${ }^{164}$.

162. SAFRAI, $1994: 274-275$.

163. Ibid. : 290-291.

164. YOUNG, $2001: 90-135$. 
In conclusion a remark needs to be made here conceming the finds of Clarias and tilapia in the Levant. In many sites these fish co-occur with Nilotic taxa such as Nile perch. On sites located far from local freshwater bodies, such as Lachish, they are considered imports from the Nile. In other cases, they are considered derived from local waters (e.g., at Tel Akko and Tell el-Oreme), or bodies of water not too distant (e.g., Megiddo, Rosh Zayit, Jerusalem and many other sites) since Clarias gariepinus and tilapia are known from the area. It cannot be excluded that these fish may have more than one origin, i.e., both "local" and imported from the Nile. It is clear from the available database that Clarias, in particular, was one of the fish taxa frequently exported from Egypt and there is no reason to suppose that it would not have been part of the "package" of fish that was traded in parts of the Levant where this fish occurred naturally. To investigate this hypothesis, mtDNA-analyses should be carried out that might allow us to identify the geographic origin of the catfish ${ }^{165}$. Such analyses are expensive, however and success depends also on the state of preservation of the ancient DNA.

A number of sites occur in which no unequivocal evidence has been found for Nilotic fish. They have yielded remains of Clarias, tilapia and cyprinids, but none of the "clear" indicators such as Lates, Synodontis, Bagrus etc. This is the case for the Iron Age Il through Byzantine levels of Tell Hesban, Nabataean Petra, Early Roman Gamla, Late Roman-Early Byzantine En Boqeq and Tamara, Roman/Byzantine Ein Gedi, and Byzantine Tel Hamid. Finally, there are a number of multi-phased sites for which the import of Nilotic fish is proven in certain periods, but not for others. The Iron Age I levels of Tell el-Oreme and Byzantine contexts of Caesarea only yielded Clarias and tilapia, and in the Byzantine levels of the Armenian monastery only Clarias was found. In younger contexts of these three sites, however, these taxa cooccur with "clear" Nilotic fish. This is also the case in an older Middle-Late Bronze Age context at Tell el-Oreme. At Tel Ashqelon, only Clarias was found in the Islamic/Crusader period, but in the older phases, this taxon was found associated with Nile perch and other Nilotic fish. It is not excluded that the continuity in fish trade at these places is obscured by the fact that the geographical distribution of certain Nilotic species such as Clarias and tilapia extends into the Levant.

165. Cf. ARNDT et al., 2003.

\section{BEYOND THE LEVANT : ISLANDS AND ANATOLIA}

The majority of exported Nilotic fish has been found in the Levantine area, but some finds are also known from the larger islands in the eastern Mediterranean and Anatolia (tables 5 and 6). Late Bronze Age finds of Nile perch have been reported from Hala Sultan Tekke on Cyprus. The Iron Age II finds of Clarias sp. at Salamis on Cyprus could either come from the Nile or the Levant, but taking into account the regular export of Nilotic fish during this period, we are inclined to see these catfish also as originating from Egypt. Later Iron Age finds of Nile perch and Clarias sp. are known from Kilise Tepe. Both at Salamis and Kilise Tepe, evidence has been found for Spathopsis ${ }^{166}$.

Even more distant from the source area are the Nile perch remains found at Miletos (Turkey) in an Archaic context. The agricultural and mineral resources of its territory, the harbours and the neighbouring sanctuary of Apollo at Didyma brought wealth to Miletos. During the $8^{\text {th }}$ and $7^{\text {th }}$ centuries BC, the regional centre established many colonies in the eastern Mediterranean, including Sinope on the Black Sea. Miletos was also involved in the creation of the trading post of Naucratis in Egypt. The trade and international contacts brought a prosperity and cosmopolitan character to the fown, which developed into a centre of Greek science and philosophy ${ }^{167}$.

Throughout the Roman-early Byzantine contexts of Sagalassos, Clarias remains are found, of which mitochondrial DNA-analysis demonstrated that they are of a Nilotic origin $^{168}$. Bagrus and Lates niloticus remains have also been found, albeit in smaller numbers. Tilapia is a fourth exotic taxon at this site, which theoretically may have come from either the Syro-Palestinian area or from the Nile. Because the three other imports have a Nilotic origin, it is likely that this is also the case for the tilapia. Circumstantial evidence indicates that the Byzantine find of Clarias from Kilise Tepe is also of Nilotic origin. The taxon has also been reported from a Flavian context at Ephesos.

The archaeological and epigraphic evidence is most substantial at ancient Sagalassos, and integrated archaeological research focused on this site has resulted in the reconstruction of an interdependent system of regional exchange mechanisms and patterns. We consider this exchange pattern as a model of why archaeological data should never be regarded at

166. REESE et al., 1986.

167. GREaVES, 2002.

168. ARNDT et al., 2003. 
Table 5 : Evidence for fish trade in Anatolian sites.

\begin{tabular}{|c|c|c|c|c|}
\hline SITE & PERIOD & TAXON & ORIGIN IN PUBLICATION & $\begin{array}{l}\text { NEW INTER- } \\
\text { PRETATION }\end{array}$ \\
\hline \multirow[t]{8}{*}{ Kilise Tepe ${ }^{a}$} & Early Bronze Age II & Sparidae indet. (seabreams) & Mediterranean $(40 \mathrm{~km})$ & \\
\hline & Early Bronze Age III & Umbrina cirrosa (corb) & Mediterranean $(40 \mathrm{~km})$ & \\
\hline & Middle Bronze Age & Carcharhinus sp. (shark) & Mediterranean $(40 \mathrm{~km})$ & \\
\hline & Late Bronze Age & Alosa fallax (twaite shad) & Mediterranean $(40 \mathrm{~km})$ & \\
\hline & Early Iron Age & $\begin{array}{l}\text { Clupeidae indet. (herrings, sardines, } \\
\text { shad) }\end{array}$ & Mediterranean $(40 \mathrm{~km})$ & \\
\hline & Early Iron Age & Katsuwomus pelamis (skipjack tuna) & Mediterranean $(40 \mathrm{~km})$ & \\
\hline & Later Iron Age & $\begin{array}{l}\text { Lates niloticus (Nile perch) } \\
\text { Clariidae (catfish) } \\
\text { Mugilidae indet. (mullets) }\end{array}$ & $\begin{array}{l}\text { Nile }(700 \mathrm{~km}) \\
\text { Nile }(700 \mathrm{~km}) \\
\text { Mediterranean }(40 \mathrm{~km})\end{array}$ & \\
\hline & Byzantine & $\begin{array}{l}\text { Clariidae (catfish) } \\
\text { Alosa fallax (twaite shad) } \\
\text { Sparidae indet. (seabreams) }\end{array}$ & $\begin{array}{l}\text { Nile }(700 \mathrm{~km}) \\
\text { Mediterranean }(40 \mathrm{~km}) \\
\text { Mediterranean }(40 \mathrm{~km})\end{array}$ & \\
\hline Milete ${ }^{b}$ & Archaic $\left(6^{\text {th }}-5^{\text {th }} \mathrm{c} . \mathrm{BC}\right)$ & Lates niloticus (Nile perch) & Nile $(850 \mathrm{~km})$ & \\
\hline Pergamonc & Late Hellenistic-Roman & Abramis brama (bream) & northwest Anatolia & \\
\hline Sagalassos ${ }^{\mathrm{d}}$ & 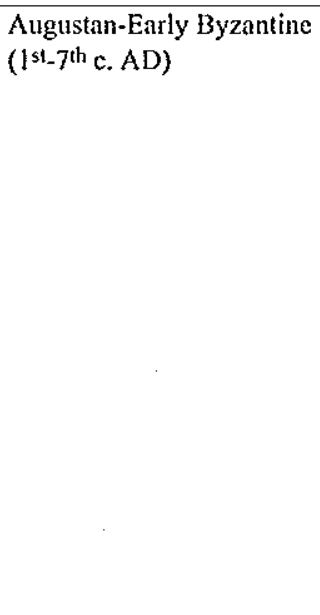 & $\begin{array}{l}\text { Lates niloticus (Nile perch) } \\
\text { Bagrus sp. (catfish) } \\
\text { Clarias gariepints (catfish) } \\
\text { Tilapinin (tilapia) } \\
\text { Epinephelus sp. (grouper) } \\
\text { Auxis rochei (bullet tuna) } \\
\text { Euthymms alletleratus (little tuny) } \\
\text { Acipenseridac (sturgeons) } \\
\text { Sardina pilchardus (pilchard) } \\
\text { Sarda sarda (bonito) } \\
\text { Scomber joponicus (Spanish mackerel) } \\
\text { Pseudophoximus handlischii } \\
\text { Cyprinus carpio (carp) } \\
\text { Leuciscus sp. } \\
\text { Vimba vimba (vinba) } \\
\text { Silurus glanis (catfish) } \\
\text { Esox lucius (pike) }\end{array}$ & $\begin{array}{l}\text { Nile }(750 \mathrm{~km}) \\
\text { Nile }(750 \mathrm{~km}) \\
\text { Nile }(750 \mathrm{~km}) \\
\text { Nile or Levant } \\
\text { Mediterranean }(120 \mathrm{~km}) \\
\text { Mediterranean }(120 \mathrm{~km}) \\
\text { Mediterranean }(120 \mathrm{~km}) \\
\text { Medit. / Black Sea } \\
\text { Medit. / Black Sea } \\
\text { Medit. / Black Sea } \\
\text { Medit. / Black Sea } \\
\text { Lake Egirdir ( } 35 \mathrm{~km}) \\
\text { outside territory Sagalassos } \\
\text { outside territory Sagalassos } \\
\text { outside territory Sagalassos } \\
\text { outside territory Sagalassos } \\
\text { outside territory Sagalassos }\end{array}$ & $\begin{array}{l}\text { Nile } \\
\text { Mediterranean } \\
\text { Mediterranean } \\
\text { Mediterranean } \\
\text { Mediterranean }\end{array}$ \\
\hline \multirow[t]{4}{*}{ Ephesose } & Hellenistic & Sander licioperca (pikeperch) & NW Anatolia & \\
\hline & Late Hellenistic & Cyprimus carpio (carp) & NW Anatolia & \\
\hline & Flavian & $\begin{array}{l}\text { Sander lucioperca (pikeperch) } \\
\text { Cyprinus carpio (carp) } \\
\text { Abramis brama (bream) } \\
\text { Rutilus frisii (kutum) } \\
\text { Clarias sp. (catfish) }\end{array}$ & $\begin{array}{l}\text { NW Anatolia } \\
\text { NW Anatolia } \\
\text { NW Anatolia } \\
\text { NW Anatolia } \\
\text { Nile or Levant }\end{array}$ & \\
\hline & Severian & $\begin{array}{l}\text { Sander lucioperca (pikeperch) } \\
\text { Cyprinus carpio (carp) }\end{array}$ & $\begin{array}{l}\text { NW Anatolia } \\
\text { NW Anatolia }\end{array}$ & \\
\hline
\end{tabular}

a. VAN NEER and WAELKENS, in press.

b. VON DEN DRIESCH, pers, comm.

c. BOESSNECK and VON DEN DRIESCH, 1985. d. VAN NEER et al., 1997 ; VAN NEER el al., 2000c ; ARNDT et al., 2003 ; VAN NEER, unpublished.

e. FORSTENPOINTNER et al., 1993, 2002. face value and how it can be integrated into a wider body of interdisciplinary evidence. Other such mechanisms may have been active in the case of Ephesos, but in this context only the economic, social, cultural, political, military and religious exchange pattern involving Sagalassos and Egypt is devel- oped ${ }^{169}$. The identification of examples of Sagalassos red slip ware, being a high quality tableware produced in the potters' quarter of Sagalassos, found at Alexandria and other Egyptian

169. POBLOME and IVAELKENS, 2003. 
Table 6 : Evidence for fish trade in sites from Cyprus and Crete.

\begin{tabular}{|c|c|c|c|c|}
\hline SITE & PERIOD & TAXON & ORIGIN IN PUBLICATION & $\begin{array}{l}\text { NEW INTER- } \\
\text { PRETATION }\end{array}$ \\
\hline \multicolumn{5}{|l|}{ Cyprus } \\
\hline \multirow[t]{2}{*}{ Hala Sultan Tekke ${ }^{a}$} & Late Bronze Age & Lates niloticus (Nile perch) & Nile $(450 \mathrm{~km})$ & \\
\hline & Roman & & & \\
\hline Apiklib & Late Bronze Age & Cyprimis carpio (carp) & Anatolia $(300 \mathrm{~km})$ & \\
\hline Salaunis ${ }^{\mathrm{c}}$ & Iron Age II and archaic & Clarias sp. (catfish) & Nile $(550 \mathrm{~km})$ or Levant & Nile \\
\hline \multicolumn{5}{|l|}{ Crete } \\
\hline Kommos ${ }^{\mathrm{d}}$ & ca. $375 \mathrm{BC}-200 \mathrm{AD}$ & Clarias sp. (catfish) & Nile $(750 \mathrm{~km})$ or Levant & Nile \\
\hline
\end{tabular}

a. ROSE, 1994.

b. DU PLAT TAYLOR, 1952 ; ROSE, 1994. c. GREENWOOD and HOWES, 1973.

d. ROSE, 1994. sites, ranging in date between early imperial and late Roman times, and the recovery of imported Nile fish at Sagalassos has been instrumental in umravelling this exchange pattern. Table wares and fish were most probably not traded for their own sake, or were at least not trend setting goods in the exchange, but good indicators, preferentially preserved in the archaeological record.

The rational exploitation of the forests around Sagalassos not only satisfied local needs, but produced a surplus that was in part traded and exported, mainly in the direction of Phrygia, but possibly also to Egypt ${ }^{170}$, and the timber may have represented the major, bulk item of the exchange. The different kinds of pitch, a secondary product of needle-leafed trees, from the Pisidian forests were most probably also the object of trade ${ }^{171}$. Whether the wool traders of Sagalassos managed to market their products further afield is unfortunately impossible to determine. The Via Sebaste linking central Anatolia with the Mediterranean coast crossed the territory of Sagalassos, and in this way the town may have profited from the maintenance taxes and transit trade of, for instance, slaves or the valued white Anatolian marbles and sarcophagi ${ }^{172}$ en route to the entire Mediterranean world, including Alexandria and Egypt. The very strong ties between Sagalassos and Pisidia at large with Egypt go back to the early and middle Hellenistic period, when the Ptolemies controlled southen Pisidia and when Pisidian mercenaries played an important role in the Egyptian army ${ }^{173}$.

170. WAELKENS and the Sagalassos team, 1997.

171. MEJGGS, 1982 ; PASZTHORY, 1988, 1990.

172. WAELKENS, 1982; WAELKENS et al., 2002.

173. WAELKENS, 2002 ; WAELKENS, in press.
The pattern of exchange between Sagalassos and Alexandria, and southern Asia Minor and Egypt in general, is corroborated when a closer look is taken at the movements of people and ideas. The incorporation of Egypt into the Roman empire was symbolized by the permanent presence of Roman military troops. Legio XXII Deiotariana was stationed at Nikopolis and was created from the troops the Galatian client-king Amyntas had deployed to pacify the towns of the Taurus, including Pisidia. Since Sagalassos together with some other towns had supported Amyntas, and the region was known by all Hellenistic rulers, including the Ptolemids, for the readiness of it mercenaries, it is probable that inhabitants of Sagalassos also served in the army of Amyntas and so also in the later legio XXII Deiotariana. The other of the two legions stationed at Nikopolis, legio III Cyrenaica, also originally consisted in a great part of Anatolian recruits of the army of Amyntas ${ }^{174}$. The unique find of a set of seven inscriptions on the rear wall of the Neon library at Sagalassos not only illustrates the mechanism of social promotion of two generations of a local noble family, but also shows that even after the reign of Augustus, Anatolian soldiers and more specifically Sagalassians still played a role in the Roman occupation force in Egypt. T. F(lavius) Attalianus Quadratus was most probably the first eques Romanus of the family, who, as praefectus cohortis II Thracum, may have served in Egypt when his unit was stationed there at some time between 86 and $105 \mathrm{AD}$. In the same period, the sister of Attalianus Quadratus, Flavia Severa, married the eques Iulius Maximianus, son of $\mathrm{M}$. I(ulius) Sanctus Maximinus. We can reconstruct the successful career of Iulius Maximianus from Egyptian papyri. These

174. DEVIJVE, 1989 ; MITCHELL, 1993 ; ALSTON, 1995. 
show that in the years 137-139 AD he was nothing less than the iuridicus Alexandreae. This was a very important position within the administration of Egypt, second in command after the praefectus Aegypti, the governor. His father, M. I(ulius) Sanctus Maximinus, also spent a large part of his military career in Egypt as tribumus of legio XXII Deiotariana, and returned to Egypt at the height of his career to serve as iuridicus Alexandreae, thus setting a precedent for his son. This term of service was probably during the reign of the emperor Domitian ${ }^{175}$. During the 1996 campaign, a new inscription was found on the lower agora. It showed that M. Gellius Maximus, who became the physician (archiatros) of the emperor Caracalla and also the procurator of the museion at Alexandria, was a native of Sagalassos ${ }^{176}$. Such officials must almost certainly have favoured closer relations between the two cities, along with the procuratores of the provincia Lycia-Pamphylia who later were appointed praefectus Aegypti.

Eastern religions in general and specifically Egyptian rites were not only tolerated by the Romans, but attracted many new worshippers throughout the empire. In Pisidia and specifically at Sagalassos indications have been found for the cult of Isis, Sarapis and Harpokrates on the local bronze coinage, decorated Sagalassos red slip ware and locally produced figirines and sculpture. The fact that the Egyptian gods took strong root in the Pamphylian harbour towns of Side, Perge, Aspendos and Attaleia might have fostered their influence in the wider region 177 .

In the light of this interdependent exchange pattern, the consumption of Nile fish at Sagalassos should not come as a surprise, but forms a nice example of how trade in fish products might reveal much more intricate exchange patterns involving the many regions of the eastern Mediterranean, which, as such, formed the structure of the ancient Mediterranean economy ${ }^{178}$. Clearly, the wider framework of the yearly Egyptian grain annona destined for Rome, for which the northern route passed along western Cyprus, southern Asia Minor and southern Crete off to Italy, was potentially beneficial to this exchange pattern, and forms another illustration of how regional fates were determined by the potential association with large-scale exchange networks, in the interest of the authorities. Whether the presence of catfish at Kommos on Crete should be explained in this way is not clear, based on the evidence of a single site. Hala Sultan Tekke on Cyprus is not

175. DEVIJVER, 1993.

176. DEVIJVER, 1996.

177. TALLOEN, 2001.

178. HORDEN and PURCELL, 2000. located within the zone of the island, which was preferentially touched by the amnona exchange, but rather in the zone associated with the northern Levant ${ }^{179}$. The results from Cyprus are also too meagre to explain in useful ways. The Ptolemaic interest in Cyprus and Rough Cilicia, disregarding the political events, may have fostered exchange patterns between these regions that were to last into Byzantine times, as evidenced by the catfish remains found at Kilise Tepe ${ }^{180}$.

In general, Nilotic fish is absent from the archaeological record of northern Levant and Syria at this time. To be sure, the royal houses of the Seleucids and Ptolemids were never close, and competed fiercely over the control of the castern Mediterranean waters. This pattern seems to continue into Roman times. Unfortunately, it is very difficult to place this negative evidence in a wider context, as Egypt was never involved in the production for export of types of artefact commonly found in the archaeological record, such as pottery, while its high quality artisanal output, mainly from Alexandria, was mostly cherished or, in the worst case, recycled for the value of its raw materials. Recently, however, this picture was confirmed by the association of a specific type of exported artefact with early Byzantine Egypt ${ }^{181}$, the so-called HIMT vessel glassi82, which is common in Egypt and the southern Levant, but does not appear in any quantity in northern Israel and other more northerly regions 183 .

\section{EXPORT OF MEDITERRANEAN FISH TO INLAND SITES}

\section{MEDITERRANEAN FISH IN EGYPT}

The evidence for Mediterranean fish in Egyptian sites is very limited, and it is not always clear whether the finds represent good indications for transport (fig. 2). Middle Kingdom to Second Intermediate Period finds (dated to 2055$1550 \mathrm{BC}$ ) are available from Tell el-Daba', currently located about $40 \mathrm{~km}$ from the coast (but it was closer in ancient times). Mediterranean fish were also found at Migdol, an Iron Age II site, dated to the $6^{\text {th }}$ century $\mathrm{BC}$, located about $15 \mathrm{~km}$ from the coast. Later finds are also limited to the Delta area

179. LUND, 1999.

180. VAN NEER and WAELKENS, in press.

181. FoY et al, 2003.

182. FREESTONE, 1994.

183. FREESTONE et al., 2002. 


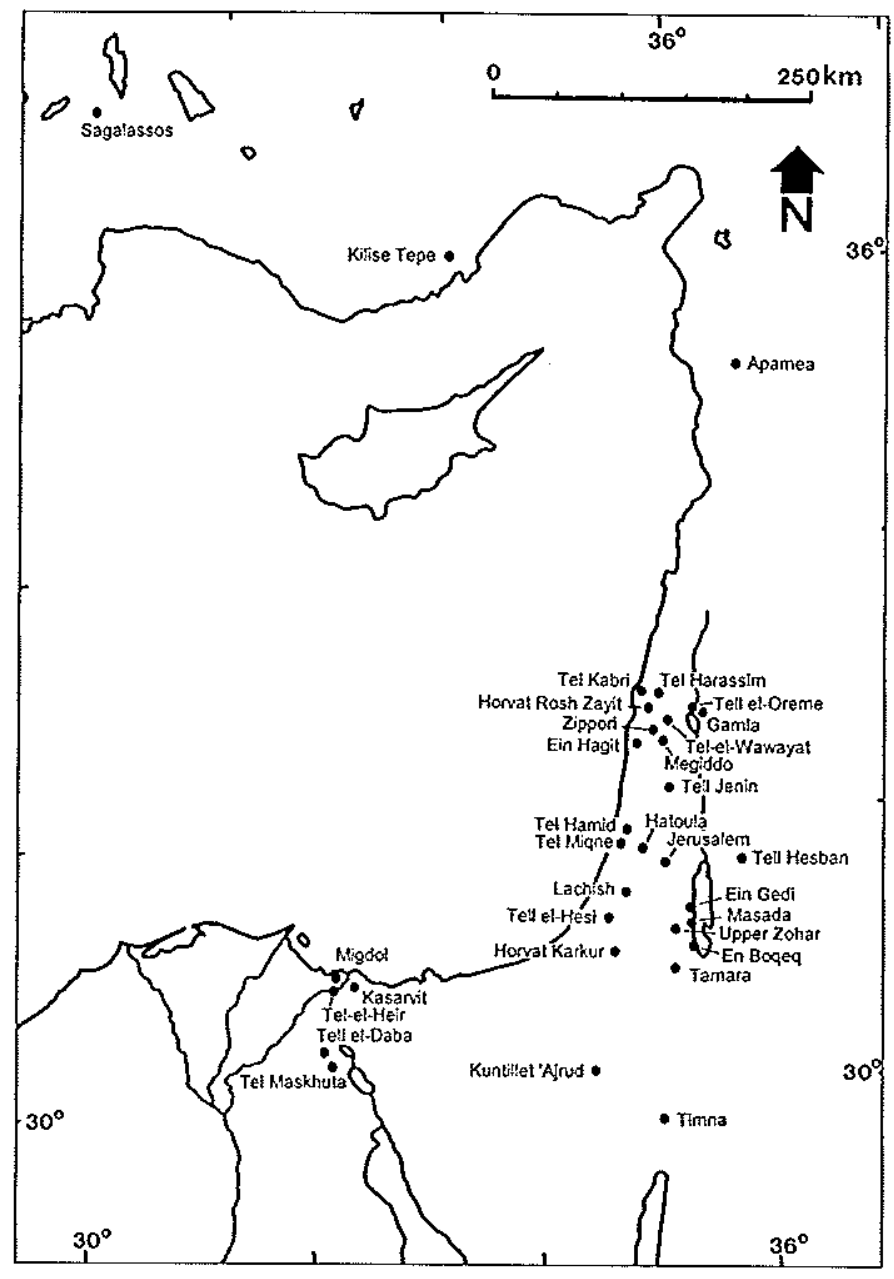

Fig. 2: Sites in the Eastern Mediterranean with indication for import of Mediterranean fish.

and include Persian/Hellenistic levels at Tel el-Heir, Late Period ( $7^{\text {th }}$ century BC) to Roman period contexts at Tel Maskhuta and Late Roman Kasarvit. These sites are located along Nile branches in the Delta at short distances from the coast, and the species found are relatively tolerant of lower salinity levels. It cannot therefore be excluded that the fish represent the local catch of specimens that entered the Delta waters rather than fish captured at sea and brought inland.

Mediterranean fish rarely figure in the archaeological record of ancient Egypt, and the available papyrological sources hardly mention sea fish. Imported marine fish did not form part of the regular Egyptian diet with plenty of fish available locally. The documents mostly mention garum or salted tunny slices, which most probably were beyond the reach of the majority of the population. The Zenon archive mentions salted fish from Byzantion, the islands of Peparethos and
Thasos, and Syria, but the dealings of Zenon were clearly in function of the higher political echelons in $3^{\text {rd }}$ century $\mathrm{BC}$ Egypt $^{184}$. In imperial times, Spanish and Tunisian garum amphorae have been identified at Mons Claudianus, but the wide range of products imported to the quarries should be seen as a consequence of the interest of the central authorities in the site, rather than representing a normal Egyptian pattern $^{185}$.

\section{MEDITERRANEAN FISH IN THE SINAI AND THE SYRO-PALESTINIAN AREA}

The oldest evidence for inland transport of Mediterranean fish comes from the Natufian and PPNA contexts of Hatoula, located at about $30 \mathrm{~km}$ from the coast. Neolithic Tel Kabri is only $5 \mathrm{~km}$ from the Mediterranean. In both cases it is obvious that the distance from the coast to the settlement was relatively small and that it could be covered in a day, making it unnecessary to preserve the fish to assure arrival of the food in a good state. The same is true for the Early Bronze Age evidence at Tel Kabri, Megiddo and Tell Jenin. Inland sites dating to the Middle Bronze Age in this area include the site of the City of David in Jerusalem and Lachish. Tel Kabri is included here as well, although the distance to the coast is only $5 \mathrm{~km}$. Late Bronze Age indications for Mediterranean fish imports are available from Tel Harassim, Tel-el-Wawayat, Megiddo and Lachish. For the Iron Age I, evidence is limited to Ein Hagit, Tell el-Oreme along the Lake of Galilee, and to the City of David in Jerusalem. Indications for the import of Mediterranean fish during the Iron Age II period are more frequent: Tel Kabri, Tel Harassim, Horvat Rosh Zayit, Megiddo, Tel Hamid, Tel Miqne, the sites of Ophel and the City of David in Jerusalem, Lachish, and Tell el-Hesi. Also the more distant sites of Tell Hesban and Kuntillet "Ajrud yielded Mediterranean species in the Iron II period.

During the Early, Middle, and Late Bronze Age periods, most Mediterranean fish appears to have been transported moderate distances from coastal ports to several inland destinations, ranging from one to three days away overland. The Early Bronze Age sites of Tel Kabri, Megiddo and Tell Jenin lie 5 to $38 \mathrm{~km}$ from the coast; the Middle Bronze Age settlements at Tel Kabri, Lachish, and the City of David (Jerusalem) are dispersed 5 to $50 \mathrm{~km}$ from the Mediterranean Sea;

184. CURTIS, $1991: 135$ and 139.

185. TOMBER, 1996. 
the Late Bronze Age towns of Tel Harassim, Tel-el-Wawayat, Megiddo, and Lachish lie 20 to $30 \mathrm{~km}$ from the sea. At most of the aforementioned sites Nile fish were also found, indicating that both Mediterranean and Nilotic fish were transported inland from coastal towns.

At Timna, during the Iron Age I period, Mediterranean fish were brought in over a relatively long distance - respectively 80 and $185 \mathrm{~km}$ (6-11 days of travel via the Negev for Timna). It is possible that Levantine merchants transported Mediterranean fish southwards to Timna following a north-south route along the eastern side of the southern Arabah (southwest Jordan). The evidence for this traffic comes from northwest Arabian pottery (Qurraya ware, formerly called "Midianite" pottery), which occurs at Timna, Amman (Jordan), and a few sites in southwest Palestine ${ }^{186}$. On the other hand, the absence of Late Bronze Age sites in the central Negev, between Timna in the southern Arabah and sites in the northern Negev, suggests that Egypt may have used another route to transport Mediterranean fish to Timna during the Ramesside period of copper mining at this site (temp. Sety I to Ramesses V) ${ }^{187}$. It is possible that Ramesside expeditions to the copper and turquoise mines in South Sinai (e.g., Wadi Maghara; Serabit el-Khadim ; Wadi Reqeita) continued overland, or by sea, further east and northwards to Timna (Egyptian Atika ?). Papyrus Harris I reveals that Ramesses III dispatched both overland caravans of donkeys and ships to Atika (identified with Timna) to obtain copperl $^{188}$. A rock-cut text of Ramesses III occurs at Site 582, $11 \mathrm{~km}$ to the northwest of the Gulf of Elat ${ }^{189}$. It may mark part of an overland route connecting Timna (19 km to the north) with the mining region in south Sinai $(110 \mathrm{~km}$ to the southwest). Ramesside expeditions may also have followed the Darb el-Hajj route across central Sinai (which also lacks sites over a distance of $190 \mathrm{~km}$ ), departing from a Ramesside fort at Kom el-Qolzoum (Suez) to reach Timna (near Elat) ${ }^{190}$. Most of the Old, Middle, and New Kingdom expeditions to south Sinai appear to have originated from northern Egypt ${ }^{191}$. In one instance, Neby, the mayor of Tjaru (Tell Heboua, near the Mediterranean Sea), commanded an $18^{\text {th }}$ Dynasty expedition to Serabit el-Khadim. Many New Kingdom expeditions would have headed southwards along the Isthmus of Suez, passing Rames-

186. HANKEY, $1995: 182$

187. COHEN, $1993: 1123-1133$; ROTHENBERG, $1993: 1475-1486$

188. PEDEN, $1994: 219$

189. ROTHENBERG, $1972: 201$, fig. 62.

190. CLÉDAT, 1916: 206-208; LECLANT, $1964: 342$ no. 11 ; MUMFORD, $1998: 545-561,1,317-318$.

191. MUMFORD and PARCAK, 2003. side shrines at Serapeum (near Lake Timsah), Gebel Abu Hassa and a Ramesside boundary stele at Gebel Mourr, to reach the southernmost Ramesside fort at Kom el-Qolzoum (Suez), before departing for south Sinai or Timna ${ }^{192}$. In the Ramesside period, these expeditions could have brought both Egyptian products and Mediterranean fish to Timna, using either a south Sinai overland route or transportation in the Red Sea (likely from Kom el-Qolzoum, or possibly from the Middle Kingdom anchorage at Wadi Gawasis, on the Red Sea coast).

In Iron Age II, there is evidence for a different dispersal pattern for the export of Mediterranean fish to the Negev. During Iron Age II, settlements reappear throughout the Negev, especially a network of forts in Iron Age IIA ${ }^{193}$. Biblical accounts generally ascribe the fortification of this region to King Solomon of Israel, who is said to have secured access to the Gulf of Aqaba and the Red Sea trade in spice and incense ${ }^{194}$. In turn, the Negev forts and spice and incense caravans provided the infrastructure by which Red Sea fish were exported northwards into the Levant (see below).

For the later periods, the evidence indicates the continued importance of Mediterranean fish in the Palestinian diet. During the Persian period, imports are attested at Tel Harassim and the City of David. At the latter site Hellenistic, early Roman and Byzantine levels also yielded such fish remains. For the early Roman period, sites with indications of Mediterranean fish include Gamla and Masada. Besides the usual food fish, the latter site has also yielded Spanish garmm amphorae ${ }^{195}$, but being one of the palaces of Herod, this evidence should not be considered typical. Three sites yielded Mediterranean fish in contexts that were only broadly dated as "Roman" or "early and late Roman" : Zippori, Megiddo and Tell Hesban. Other broadly dated indications are the late Roman-early Byzantine finds from En Boqeq and Tamara, and the Roman-Byzantine material from Ein Gedi. For the early Byzantine period, Upper Zohar presents the only evidence. Finds labelled as "Byzantine" or "early and late Byzantine" are known from Tel Harassim, the City of David, Tell Hesban and Horvat Karkur. Early Islamic finds, finally, are known from the Armenian monastery in Jerusalem.

Clearly, the evidence for the consumption of Mediterranean fish is most abundant in Palestine. This is partly a result of the tradition of research in the area, but it does form a meaningful contrast to the Egyptian situation, for instance.

192. CLÉDAT, $1916: 206-208$; MUMFORD, $1998: 1,317-318$.

193. COHEN, $1993: 1123-1133$.

194. 2 Chronicles $8: 17-18$; MUMford, $1998: 1,397-398$.

195. CotTon et al., 1996. 
Egypt was mostly self-sufficient in its fish needs, and even managed to export its fish products. Palestine, on the other hand, had a rich fish supply in the Sea of Galilee, but this was not sufficient to cover all its needs. The Mediterranean, which was never far away, represented an obvious additional source, and when handled correctly, sea fish was kosher. No specific pattern can be read in the evidence to allow the reconstruction of the trade in sea fish, except that Mediterranean fish is attested in a wide variety of contexts. As a result, we are dealing with a constant need for a staple good, implying that sea fish formed a normal commodity in daily transactions, but this is mostly beyond the reach of archaeology.

The presence of grouper bones in an early Byzantine context at Apamea is a piece of isolated evidence that demonstrates that sea fish formed a supplement to the catfish from the floodplain of the Orontes. It is striking that the marine fish only occurs in contexts predating the $613 \mathrm{AD}$ invasion of the Persians. Afterwards, the local elite disappeared, and import of sea fish apparently also stopped ${ }^{196}$. Amphorae attest to the importation of Spanish fish products to the coastal towns of Syria in the second and fourth centuries AD. In mid imperial and early Byzantine times, the import of amphorae with fish products (?) from the Black Sea is also attested ${ }^{197}$.

\section{MEDITERRANEAN FISH IN ANATOLIA}

The evidence for inland transportation of Mediterranean fisl into Anatolia is limited to two sites only, no doubt because fish remains have received little attention thus far in this region. At Kilise Tepe, located along the Göksu basin, about $40 \mathrm{~km}$ from the coast, all the occupation levels with fish remains yielded evidence for the importation of marine fish. This is the case for Early Bronze Age II, Early Bronze Age III, Middle Bronze Age, Late Bronze Age, Early Iron Age and Later Iron Age contexts. The transport over land probably took a day or two, but it is unclear if the Mediterranean fish were cured for the purpose of transport ${ }^{198}$. In the case of Sagalassos, such must have been the case, as the site is located about $120 \mathrm{~km}$ from the Mediterranean. Here, Mediterranean fish occurred in contexts dating from Augustan to early Byzantine times. Commercial relationships with neighbouring coastal Pamphylia are well documented archaeologically ${ }^{199}$.

196. VAN NEER, 1984.

197. BUTCHIER, 2003 : 196-199.

198. VAN NEER and WAELKENS, in press.

199. BRANDT, 1992.
From the study of the many stray coins found during the excavations at Sagalassos, it is clear that the major Pamphylian harbours of Side, Aspendos, Perge and Attaleia were already in contact in late Hellenistic times. From the second century $A D$ onwards until the end of the civic coinage in the second half of the next century, Perge features strongly in the numismatic evidence, while the other southern harbour cities have a more token presence. The coins from Perge in second century and Severan Sagalassos indicate the importance of the good link with this harbour town, from where the entire Mare Nostrum was accessible and the region of Sagalassos could interlock with wider exchange patterns ${ }^{200}$. It should come as no surprise that a fair amount of early imperial to early Byzantine Sagalassos red slip ware has been identified at Perge 201 , and that among the imported pottery items at Sagalassos the lead glazed tableware made at Perge is represented. The proposed presence of a "Bauhuitte" from Perge involved in the construction of the Hadrianic Nymphaeum north of the Lower Agora ${ }^{202}$ may have had a more considerable economic impact. The activities of an architect from Perge at Sagalassos have also been attested epigraphically. In the next period, both Pamphylia and Pisidia were promoted as a result of their contribution in the provisioning of the military campaigns in the cast, with Side growing in importance especially upon the installation of the Roman fleet in its harbour, a relationship reflected in the "Homonoia" coins of Sagalassos with Side ${ }^{203}$. After that, the epigraphic habit disappears and civic coinage is discontinued. The fish remains indicate, however, that exchange with Pamphylia continued until the abandonment of the town in the middle or second half of the $7^{\text {th }}$ century AD.

\section{TRADE IN RED SEA FISH}

\section{INSIDE EGYPT}

Of course, Egypt also made use of the trade potential offered by the Red Sea, and this natural connection grew into a substantial branch of eastem trade in Roman times ${ }^{204}$. The Ptolemies had already invested in the establishment of the required port facilities and fortified desert road connections

200. SCHEERS, 2000.

201. ATIK, 1995.

202. VANDEPUT, $1997: 89-95$

203. WAELKENS, 2002.

204. YOUNG, $2001: 27-89$. 
reaching the Nile, through which a growing variety of luxury goods, medicines and incenses used in religious rites were imported from India, Arabia and Africa. Fish products from the Red Sea found their way into Egypt in the same way as the Nile fish reached the Red Sea coast, taking advantage of the official interest in wider ranging exchange patterns and the infrastructure of distribution. The finds are of early imperial or later date and are related to the transports that took place along the desert routes 205 . Some of the sites are along the desert trade roads or in the neighbourhood of Coptos, where the Romans concentrated, controlled and taxed the Red Sea transit traffic en route to Alexandria, where the goods could be put on sale, processed into artisanal products or transhipped into the Mediterranean. Scaridae are the only taxon reported from $2^{\text {nd }}$ century $A D$ refuse deposits of El-Zarkeh, a desert station along the Qift - Quseir al-Qadim road, about $65 \mathrm{~km}$ from the coast, and it was also the predominant taxon in the late first - mid-third century AD site of Mons Porphyrites, located about $50 \mathrm{~km}$ from the coast along the Abu Sha'ar - Nile road. In Byzantine (Coptic) levels of Shenhur, in the area of Coptos in the Nile Valley, parrotfish have also been reported (fig. 3).

Fish remains formed part of the diet supplied to the workforce at the imperial stone quarries, the road connections of which were later developed for the Red Sea trade as well. There is evidence from ostraca that first-second century AD Mons Claudianus received food supplies from the Nile Valley ${ }^{206}$, but the faunal remains show that Red Sea molluscs and at least 12 families of Red Sea fish were also brought in. The distance of this quarry site to the coast is about $50 \mathrm{~km}$, which would make it feasible to bring in marine products in fresh state during the cooler months of the year. It is striking that at all sites the parrotfish are very numerous, a fish family that is very suitable for drying and that is still frequently commercialized in that form. It is therefore likely that dried Red Sea fish was brought to Mons Claudianus.

\section{TO THE SYRO-PALESTINIAN AREA}

The oldest evidence in the Levant for trade in Red Sea fish comes from the Iron Age II period, which represents a time of resettlement and fortification of the caravan routes in the

205. SIDEBOTHAM and ZITTERKOPF, 1995.

206. TOMBER, 1996.

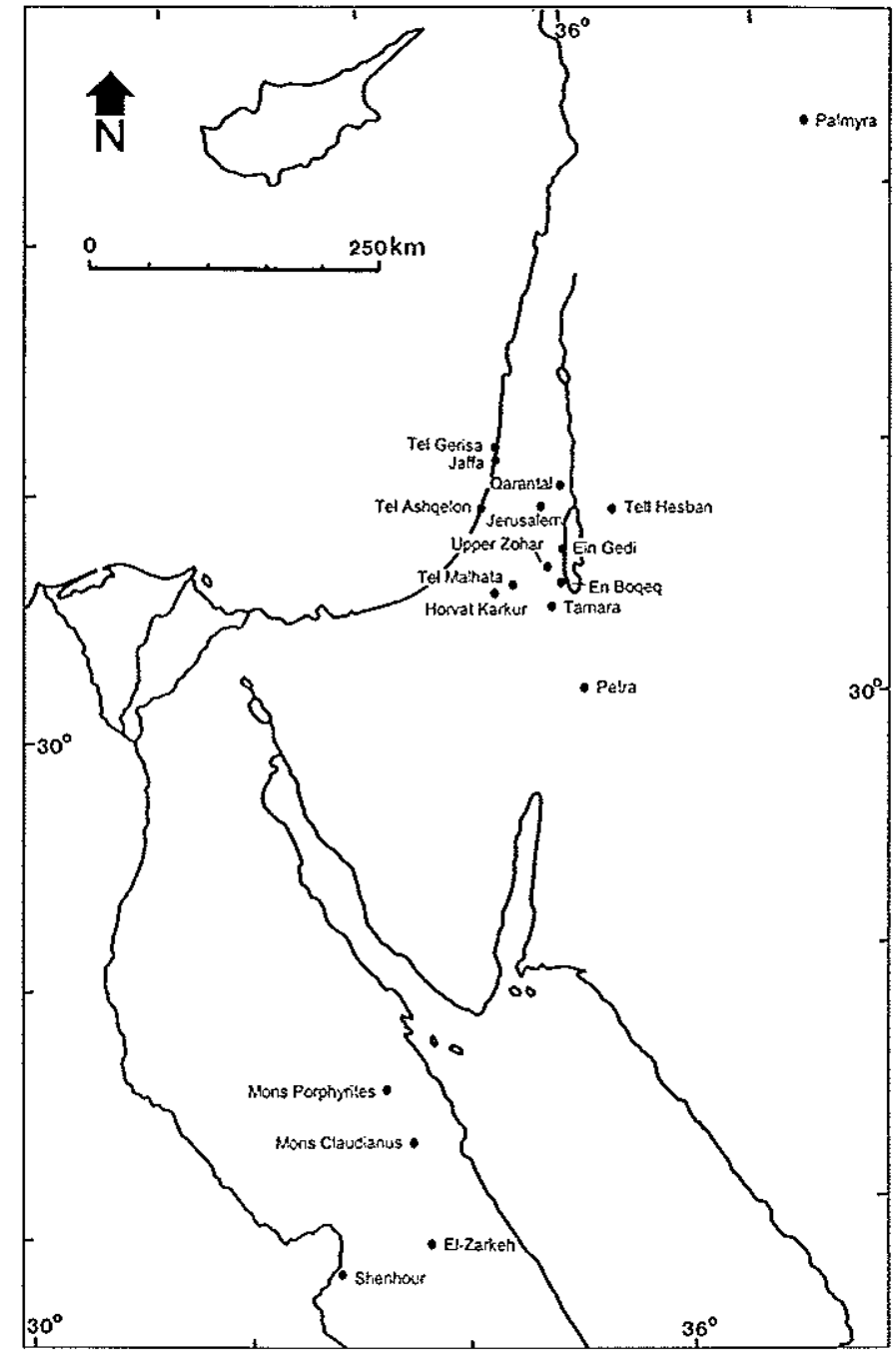

Fig. 3 : Sites in the Eastern Mediterranean with indication for import of Red Sea fish.

Negev 207 in order to attain access to the Red Sea incense and spice trade. During this time, Red Sea parrotfish appear at Tel Ashqelon, Tel Gerisa, and Tell Hesban, which lie 225, 280, and $250 \mathrm{~km}$, respectively, from the Red Sea. These distances represent $7-13$ days, 9-17 days, and $8-15$ days of overland transportation, respectively. The distribution of these Red Sea fish reflects the transportation of spices and incense by overland caravans from Arabia and the Red Sea, moving through the Negev to the coastal Plain of Sharon (Philistia and Israel) and heading northwards through western Jordan to Syria. At different points throughout the Iron Age II period, Israel, Judah, Edom, and Assyria built or maintained forts and way-

207. COHEN, $1993: 1123-1133$. 
stations in the Negev (e.g., Tell Jemmeh; Kadesh Barnea; Tell el-Kheleifeh), securing this region for caravan traffic and Red Sea shipping208.

Aside from Sheshonq I's invasion of the Negev, Egypt did not play a direct role in the Red Sea trade. Egyptian(izing) artefacts remain either unpublished or absent from Period I ( $10^{\text {th }}$ or $9^{\text {th }}$ centuries BC) and Period II ( $8^{\text {th }}$ century BC) at Tell el-Kheleifeh, but amount to approximately $10 \%-20 \%$ in Period III ( $c a$ 783-733 BC) and 7\%-15\% in Period IV ( $c a$ 700 ?-586 BC); these artefacts include calcite vessels, jewellery (beads; amulets), and sealings ${ }^{209}$. Despite significant percentages of Egyptian products and influence in the Negev (e.g., hieratic ostraca), the Assyrian and Neo-Babylonian empires controlled the Red Sea trade during late Iron Age II. For example, in pottery assemblages from the Negev and adjacent regions, Assyrian-derived pottery amounts to $10 \%$ at Horvat Qitmit (ca 650-586 BC) and 14\%-20\% in phase iii at Tavilan (Area II phases ii-iv : 6\%, $14 \%, 17 \%$; Area III phases i-iv : $5 \%, 7 \%, 20 \%, 9 \%$; $c a 675-500 \mathrm{BC})^{210}$.

At Petra, located at about $100 \mathrm{~km}$ from the Gulf of Aqaba, at least 10 Red Sea taxa have been found in Nabataean levels, and 9 taxa in Late Roman contexts. Early and Late Roman contexts from Tell Hesban also indicate commercial links with the Gulf of Aqaba. At Karantal, finally, the Red Sea fish were from levels dated as "Roman". Red Sea fish has also been attested in Late Roman-Early Byzantine contexts from En Boqeq and Tamara. More broadly dated finds are known from Roman/Byzantine levels at Ein Gedi and Tel Malhata. For the Early Byzantine period, Red Sea fish have been attested at Palmyra, Upper Zohar and Horvat Karkur. Finds listed as "Byzantine" are known from Tel Ashqelon and the Armenian monastery in Jenusalem. Those sites also yielded Red Sea fish in their Early Islamic levels, and at Tel Ashqelon these fish were also found in the later Islamic/Crusader period. Crusader period parrotfish finds exist from Jaffa. The aforementioned finds show that Red Sea fish might have become part of another large-scale trade connection, supplying the ancient Mediterranean with southem Arabian aromatics 211 . This link pre-dated Roman influence in the area, and mainly the Nabataeans are known to have exploited the poten-

208. MUMFORD, $1998: 1397-1404,1416-1429$.

209. GLUECK, $1939: 13,17,20$; HERRMANN, $1994: 10,50$ no. 458, 18, 50 no. 883 ; MUMFORD, 1998 : 1397-1404, 1416-1429; PRATICO, 1985 : 2627.

210. MUMFORD, $1998: 1,429-431,1,441$; BENNETT and BIENKOWSKI, $1995: 61-66$, data tables 6.1-6.3 and 6.6-6.12 ; BEIT-ARIEH, $1995: 212$ no. 5 ; 214 , table 4.1 .

211. YOUNG, $2001: 90 \cdot 135$. tial of the caravan routes crossing the Arabian peninsula and the harbour of Leuke Kome. The trade routes converged at Petra and finally reached the Mediterranean at Gaza. From the port of Gaza or overland, a part of the merchandise passed into Alexandria and Egypt, while some of the aromatics were also directed north from Petra towards the Decapolis and Damascus. The Petra-Gaza connection, and the Arabian trade in general, continued upon the Roman instalment of the provincia Arabia into the Severan period, but seems to have suffered from the added-on effects of the third century crisis. In late Roman times, part of the Red Sea trade was diverted from Egypt towards Aila, while Bostra capitalized on part of the Palmyrene caravan routes, but the overall importance of the Arabian incense and eastern trade had declined.

The Arabian incense trade provided another scenario by which regional products of relatively unimportant economic impact could profit from the association with a wider-ranging network of distribution. Red Sea fish supplemented the already attested need for fish imports into the Lands of Israel. It is striking, however, that the distribution of Red Sea fish in the southern Levant increased in importance at a later stage compared to Nilotic fish imports into the same area. It is not clear at this point whether this is related to the archaeozoological evidence available or whether the Arabian incense trade, which indirectly promoted the distribution of Red Sea fish, became more rooted in the general area of the southern Levant at a later stage, possibly upon the annexation of Roman Arabia, when this trading scheme gradually loses its importance. Possibly the wider distribution of Red Sea fish, coupled with a fair amount of imported Nile fish, in later Roman times, might simply be linked to the general economic well-being of the contemporary Palestinian economy ${ }^{212}$. Once again, the variety of sites in the region, at which Red Sea fish was attested, indicate that this commodity might have become part of daily transactions in the urban and rural markets, adding variety to the traditional diet.

Apparently, the mechanisms by which Red Sea fish reached the southern Levant, and even Palmyra, were still partly in place during and after the end of the Roman hegemony over the region. In general, the contemporary archaeological record is less well known, but this type of evidence could indicate some form of continuity of inter-regional trade patterns in early Islamic times. The Dome of the Rock in Jerusalem formed part of a building programme along with the alAqsa mosque, one of the earliest Islamic monuments, and a palatial or governmental complex at the foot of the Mount,

212. KINGSLEY, 2001 
representing the continued prosperity of the region. Ashqelon and Jaffa provided the Crusaders of Jerusalem access to the Mediterranean, while the fall of Shobak in 1115 extended their territorial control to the Red Sea.

\section{TRADE IN ANATOLIAN FRESHWATER FISH}

Evidence for the trade in Anatolian freshwater fish is limited to four sites thus far, no doubt because of the lack of attention paid in the past to the recovery of smaller animal bones in this area. The most striking find is the carp (Cyprimus carpio) found at Apikli on Cyprus, which dates to the Early Bronze Age. Contacts between Anatolia and Cyprus existed since the $9^{\text {th }}$ millennium $\mathrm{BC}^{213}$, but a more regular pattern of contacts, influence and presumably immigration from Anatolia is attested from the Early Bronze Age onwards, possibly triggered by patterns of change induced by the contemporary early state formation processes and the arrival of the IndoEuropeans on the scene. From then on, its metal resources placed the island on the map and, given its strategic location in the eastem Mediterranean, gradually started to determine the island's development.

The major food fish at Roman-early Byzantine Sagalassos was carp, which is a species that could not live in the waters around the site, and this was the case for all the fish taxa found at the site. Thus far, it has been impossible to establish the exact origin of the wild carp, since it had a wide distribution. The closest locality where it might have come from is Lake Eğirdir, but also Lakes Beyşehir, Eber and possibly Akşehir may have been the source for wild carp, as well as one of the distant larger rivers, such as the Büyik Menderes or the Gediz to the west, or the Sakarya to the north. Pike, a species found in rather low quantities at Sagalassos, may also have been derived from various waters : Lakes Eber and Akşehir, the Büyük Menderes, Sakarya or Kizil Irmak rivers, or lakes in the Marmara region. Unequivocal evidence for the import of fish from Lake Egirdir is given by Pseudophoximus handlirschii, which is endemic to that lake. Now the species is close to extinction because of the introduction of pikeperch, but it used to be a major food fish that was also prepared for export in the first half of the twentieth century ${ }^{214}$. Trade of salted, sinilar sized cyprinids from the nearby Beyşehir lake also occurred ${ }^{215}$. Obviously,

213. VIGNE and BUITENHUIS, 1999.

214. Ünlüsayın, pers, comm.

215. NÜMANN, 1961. the regional exchange patterns in which Sagalassos was involved did not only include Pamphylia, the Mediterranean and Egypt, but reached inland Anatolia as well. The pattern of inland exports of Sagalassos red slip ware overlap very nicely with the identification of other city coins found at Sagalassos, and both categories of evidence indicate contacts and exchange with communities and regions in western and north-western Asia Minor, along with the importance of the connecting road network ${ }^{216}$. The import of statuary from Dokimeion and Aphrodisias to the Pisidian primus inter pares confirms this picture ${ }^{217}$, and the newly defined pattern may serve as an illustration of the role of overland exchange in Asia Minor. More research is needed to understand the other aspects involved in this inland exchange pattern, but fish products and the other attested artefact categories once more found a wider distribution through association with a wider-ranging platform.

The bream (Abramis brama), which is found today in the lower reaches of the Sakarya river and some other waters in the extreme north-western part of Anatolia is attested outside its natural distribution in Pergamon, in contexts dated as late Hellenistic-Roman, and at Ephesos in Flavian levels. Roman contexts from Ephesos yielded also remains of Rutilus frisii, another good indicator of long distance trade with northwest Anatolia. That species has a distribution more or less similar to that of bream, and is found in addition in Lake Iznik. Pikeperch (Sander lucioperca) was also found at Ephesos that must have come from northern Anatolia. Cities such as Pergamon and especially Ephesos were involved in multifaceted exchange patterns with a multitude of regions in the wider Mediterranean, exemplified by the export pattern of Pergamene sigillata ${ }^{218}$ and the variety of amphorae imported to Ephesos 219 . The attested import of fish from north-western Anatolia adds a new element, but may come as no surprise. After all, Pliny praised the Sangarios river basin not so much for its size, but for its enormous supplies of fish.

\section{CONCLUSIONS}

The presence of fish remains belonging to species that do not occur in the region of a given archaeological site is a useful indicator of former trade comnections, as has been

216. POBLOME, 1999 : 288 ; SCHEERS, 2000.

217. MOENS et al., 1997.

218. MEYER-SCHLICHTMANN, 1988.

219. BEZECKY, 2001 
demonstrated above by bone finds of fish from the Nile, the Mediterranean, the Red Sea and Anatolia. Comparison of the faunal data with archaeological, artefactual and - when available - textual data seems to indicate that the number of sites with exotic fish reflect general patterns in the commercial relations between producer and consumer areas, although poor dating of the fish remains sometimes hampers the interpretation. The dataset indicates that the export of Nilotic fish has the longest tradition, starting in the Late Chalcolithic and Early Bronze Age with few finds in more southerly located coastal settlements of the Levant. From the Middle Bronze Age onwards finds become more numerous and also occur inland and on more northerly located coastal sites. During the Iron Age, Nilotic fish appear not only at a greater number of sites but also at a wider range of large to smaller ports and inland settlements, possibly reflecting the access of a larger portion of the population to items from Egypt. The documented trade mechanisms include transport by ship combined with the interaction of coastal sites with their hinterland, as well as directed caravan trade to specific destinations. It is striking that most inland sites in the Levant with Mediterranean fish also yield Nilotic fish, indicating that the latter probably arrived by ship at the coastal towns from where they were transported inland along the same roads as the Mediterranean fish. Evidence for trade of Red Sea fish dates exclusively to the Iron Age II and later periods, showing that these species were transported over land once the caravan routes in the Negev were fortified and when the region gained access to the Red Sea incense and spice trade. Evidence for this trade continues until the Byzantine period, and the same holds true for Mediterranean and Nilotic fish trade in general.

In several instances, e.g., for the Middle Bronze Age and the Iron Age period, fish bones are the only indicators available on a site for contacts with other regions. The careful recovery and analysis of fish bones from Eastern Mediterranean sites should allow this find category to become an even more powerful tool in the documentation of former trade comections. In addition, it needs to be verified in the future to what extent the presence of exotic fish is a marker of identity or status 220 . This will only be possible when ichthyofaunas become available from a wider variety of settlements, and are studied in an interdisciplinary way.

\section{ACKNOWLEDGEMENTS}

Ve would like to thank L. Bartosiewicz, A. von den Driesch and J. Peters, S. Hamilton-Dyer, E. Maher and M. Sade for providing us with unpublished data. The contribution of Wim Van Neer, Jeroen Poblome and Marc Waclkens to this text presents research results of the Interuniversity Attraction Poles Programme - Belgian Science Policy, of the Conserted Action of the Flenish Government (GOA 97/2) and of the Fund for Scientific Research-Flanders (Belgium) (FWO, G.2145.94).

WiII VAN NEER Royal Museum for Central Africa Lewconsesteenveg 13 B-3080 Terviren Belgitum vanneer@africamuseum.be

OIIII LERNAU

2 Nurit POB 1268

Mevisseret Zion Israel

ozlernan@netvision.ut.til

Renée FRIEDMAN

Deparment of Ancient Egypt and Stulan British Museum

London J'C1B 3DG

reneefavirgin.net

Gregory MUMFORD

Department of Near and Middle Eastern Civilizations

University of Toronto

CANADA M5S 1CI

gregorymmmford@hormail.com.

Jeroen POBLOME and Marc WAELKENS

Katholieke Universiteit Lenven

Department of Archaeology Blijde Inkomststrant 21 B-3000 Lewven Belginm

jerocn.poblome@arts,kulemven,ac,be marc.wnelkens@arts.kulewven.ac.be

220. VAN NEER and ERVYNCK, 2004. 


\section{BIBLIOGRAPHY}

ALSTON R.

1995 Soldier and Socicty in Roman Egypt. A Social History. London : Routledge.

ALTENSELLER $\mathrm{H}$.

2001 Trade and Markets. In : REDFORD D.B. (ed.), The Oxford Encyclopedia of Ancient Egypt 3:445-450. Oxford : Oxford University Press.

AL-Zaivahra M.A.M.

1999 The Fanal Remains from Tell Jenin, Northern West BankPalestine. Leuven : Katholicke Universiteit, M.A. Thesis.

ARNDT A., VAN NEER W., HELlemans B., RobBen J., Volckaert F. and WAELKENS $\mathrm{M}$.

2003 Roman trade relationships at Sagalassos (Turkey) elucidated by ancient DNA of fish remains. Joumal of Archueological Science $30: 1095-1105$.

ARNOLD D., ARNOLD F. and ALLEN S.

1994 Canamite imports at Lisht, the Middle Kingdom capital of Egypt. Agypten und Levante 5 : 13-32.

ATIK $N$.

1995 Die Keramik aus den Südthermen von Perge. lstambuler Mitteilungen. Beiheft 40:1-211. Tübingen : Ernst Wasmuth.

BARNET R.D.

1982 Ancient lories in the Middle East and Adjacent Countries. Jerusalem : The Hebrew University of Jerusalem (Qedem 14, Monographs of the Instinte of Archaeology).

BAR-YOSEF MAYER D.E.

2001 Trate in the Late Bronze Age and lron Ages as reflected by the shells of Lachish, Megiddo and Beth Shean. Preliminary Abstract Book, American Sclool of Oriental Research Annual Meeting.

2002 Egyptian-Canaanite Interaction during the Fourth and Third Mitlennia BCE : The Shell Connection. $m:$ LEVY T.E. and VAN DEN BRINK E.C.M. (eds), Egypt and the Levant. Interrelations from the $4^{\text {th }}$ through the early $3^{\text {rd }}$ Millemitm $B C E$ : 129-138. London : Leicester University Press.

BEDFORD P.R.

2001 Temple Restoration in early Achaemenid Judah. Joumal for the Study of Judaism. Supplement 65. Leiden : Brill.

\section{BEIT-ARIEH I.}

1995 Hon at Qitmit : An Edomite Shrine in the Biblical Negev. Tel Aviv: Institute of Archacology of the Tel Aviv University (Monograph Series of the Instilute of Archaeology of the Tel Aviv University 11).

BENNETT C.-M. and BIENKOWSKI P.

1995 Excavations at Tawilan in Southern Jordan. Oxford : Oxford University Press.

BERLIN A.M.

1992 Hellenistic and Roman Pottery. Preliminary Report, 1990. In: VANN R.L. (ed.), Caesarea Papers. Straton's Tower, Herod's Harbour, and Roman and Byzantine Caesarea. Journal of Roman Archaeology' Stpplementary Series 5: 112 128. Ann Arbor.

BEZECZKY T.

2001 Late Hellenistic - early Roman amphora stamps from Ephesus. $I n$ : KRINZINGER F. (Hrsg.), Studien zur hellenistischen
Keramik in Ephesos. Ergänzungshefie zu den Jahresheftes des Österreichischen Archäologischen Institutes in Wien 2 : 11-19. Wien.

BIETAK M.

1997 Avaris, capital of the Hyksos Kingdom : new results of excavations. In : OREN E.D. (ed.), The Hytsos : New Historical and Archaeological Perspectives: 87-140. Philadelphia: University of Pennsylvania Museum.

BIGELOW H.B. and SCHROEDER W.C.

1953 Fishes of the Westem North Atlanic. Sanfishes, Guilar. fishes, Skates and Rays, Chimaeroides. New Haven : Sears Foundation for Marine Research (Memoir Sears Fomdation for Marine Research 1,2$)$.

BLACHE J. et MITON F.

1962 Première Contribution à la Connaissance de la Pêche duns le Bassin Hydrographique Logone-Chari-Lac Tchud. Paris : ORSTOM.

BOESSNECK J.

1977 Die Tierknochenfunde aus Isan Bahriyat. In: HROUDA B. (Hrsg.), Isin-Isam Bahriyat I : 111-133. München : Verlag der Bayerischen Akademic der Wissenschaften (Bayerische Akademie der Wissenschaften, Philosophisch-Historische Klasse, Abhandlungen - Nette Folge 79).

1992 Besprechung der Tierknochen- und Molluskenreste von Hassek Höyük. Istanbuler Forschungen 38 : 58-74.

BOESSNECK J., VON DEN DRIESCH A. und STEGER U.

1984 Tierknochenfunde der Ausgrabungen des Deutschen Archaeologisches Institut, Baghdad, in Uruk-Warka. Baghdater Mitteilungen $15: 149-189$.

BOESSNECK J. und VON DEN DRIESCH A.

1985 Knochenfunde aus Zisternen in Pergamon. München : UniDruck.

BOESSNECK J. und ZIEGLER R

1987 Tierknochenfunde III. Serie 1983-1984 (7.-8. Kampagne). In : Hrouda B. (Hrsg.), Isin-Isan Bahriyat III. Die Ergebnisse der Ausgrabungen 1986-1989: 137-150. München: Verlag der Bayerischen Akademie der Wissenschaften (Bayerische Akademie der Wissenschaften, Philosophisch-Historische Klasse, Abhandlingen - Newe Folge 94).

BOULENGER

1907 Zoology of Egypt. The Fishes of the Nile, London : Hugh Press.

BRANDT H.

1992 Geselschaft und Wirtschaf Pamphyliens und Pisidiens im Altertum. Asia Minor Studien 7. Bonn : Habelt.

BUNIMOVITZ S

1995 On the edge of empires - Late Bronze Age (1 500-1 200 BCE). $n$ : LEVY T.E. (ed.), The Archaeology' of Society in the Holy Land: 320-330. London: Leicester University Press.

BUTCHER K.

2003 Roman Syria and the Near East. Los Angeles : Getty Publications.

ClÉdat J.

1916 Notes sur l'Isthme de Suez. Bulletin de l'Institut Français d'Archéologie Orientale 16: 201-228. 
COHEN R.

1993 Middle Bronze Age I and Iron Age ll sites in the Negev hills. In: STERN E., LEWINSON-GILBOA A. and AVIRAM J. (eds), The New' Encyclopedia of Archaeological Excavations in the Holy Land $3: 1123-1133$. Jerusalem: The Israel Exploration Society.

COTTON H.M., COCKLE W.E.H. and MILLAR F.G.B.

1995 The papyrology of the Roman Near East : a survey. The Journal of Roman Studies $85: 214-235$.

COTTON H., LERNAU O. and GOREN Y.

1996 Fish sauces from Herodian Masada, Journal of Roman Archueology $9: 223-238$.

CURTIS R.I.

1991 Garum and Salsamenta. Production and Commerce in Materia Medica. Studies in Ancient Medicine 3. Leiden : Brill.

DAVIES N. DE G. and FAULKNER R.O.

1947 A Syrian Trading Venture in Egypt. Journal of Egyptian Archaeology $33: 40-46$.

DE GROSSI MAZZORIN J.

2000 État de nos connaissances concemant le traitement et la consommation du poisson dans l'antiquité à la lumière de l'archéologie. Mélanges de l'École Française de Rome, Antiquité 112 : 155-167.

DESSE-BERSET $\mathbf{N}$. and STUDER $\mathbf{j}$.

1996 Fish remains from ez Zantur (Petra, Jordan). $m:$ STUCKY R.A. (ed.), Terra Archaeologica II, Monographien der Schweizerisch-Liechtensteinischen Stiffung für Archäologische Forschungen im Ausland: 381-387. Mainz: Philipp von Zabern.

DEVIJVER $\mathrm{H}$.

1989 L'Égypte et l'histoire de l'armée romaine. $m$ : CRISCUOLO L. i GERACI G. (a cura di), Egitto e Storia Antica dall'Ellenismo all'Eta Araba: 37-54. Bologna : Cooperative libraria universitaria.

1993

The inscriptions of the Neon-Library of Roman Sagalassos. In: WAELKENS M. and POBLONE J. (eds), Sagalassos II Report on the Third Excavation Campaign 1992: 107-123. Leuven: Leuven University Press (Acta Archaeologica Lovaniensia Monographiae 6).

1996 Local elite, equestrians and senators : a social history of Roman Sagalassos. Ancient Society 27 : 105-162.

DREXHAGE H.-J.

1993 Garum und Ganumhandel im römischen und spätantiken Ägypten. Mï̈nstersche Beiträge zur antiken Handelsgeschichte $12: 27-55$.

DU PLAT TAYLOR J.

1952 A Late Bronze Age settlement at Apikli, Cyprus. Antiquaries Jounal $32:$ 133-167.

DUSINBERRE E.R.M.

2003 Aspects of Empire in Achaemenid Sardis. Cambridge : Cambridge University Press.

ELAIGNES.

1998 Alexandrie : étude préliminaire d'un contexte céramique du Haut-Empire. Alexandrina $1: 75-114$.

ELLISON R., RENFREW J., BROTHWELL D. and SEELEY N.

1978 Some food offerings from Ur, excavated by Sir Leonard Woolley and previously unpublished. Joumal of Archaeological Science 5 : 167-177.
ENGELS D.W.

1978 Alexander the Great and the Logistics of the Macedonian Army. Berkeley : University of California Press.

EMPEREUR J.-Y. (éd.)

1998 Commerce et Artisanat dans l'Alexandrie Hellénistique et Romaine. Bulletin de Correspondance Hellénique Supplément 33. Paris : De Boccard.

FISHER M.

1989 An Early Byzantine settlement at Kh. Zikrin (Israel). A contribution to the archaeology of pilgrimage in the Holy Land. $m$ : Actes du XI' Congrès international d'Archéologie chrétienne (Ljon, Vienne, Grenoble, Genève et Aoste, $21-28$ septembre 1986): 1787-1807. Rome: École Française de Rome-Pontificio Istituto di Archeologia Cristiana.

2002 Yavnel-Yam 1992-1999-Interim report. Qudmoniof $25: 2-11$. FORSTENPOINTER G., SCHERRER P., SCHULTZ O, and SATTMANN H.

1993 Archäologisch-paläoanatomische Untersuchungen an einer hellenistischen Brumenanlage in Ephesos, Türkei. Wiener tierärtzliche Monatsschrift $80: 216-224$.

FORSTENPOINTNER G., WEISSENGRUBER G. and GALIK A.

2002 Banquets at Ephesos; archaeozoological evidence of well stratified Greek and Roman kitchen waste. $I n$ : BUITENHUIS H., CHOYKe A.M., MASHKOUR M. and AL-ShIYAB A.H. (eds), Archaeozoology' of the Near East V. Proceedings of the fifh international symposim on the archueozoology of southwestern Asia and adjacent areas: 282-304. Groningen and Yarmouk University : ARC-Publicaties 62.

FOY D., PICON M., VICHY M. et THIRION-MERLE V.

2003 Caractérisation des verres de la fin de l'Antiquité en Méditerranée occidentale : l'émergence de nouveaux courants commerciaux. $I n$ : FOY D. and NENNA M.-D. (éd.), Échanges et Conmerce du Verre dans le Monde Antique: 4I-85. Montagnac : Éditions Monique Mergoil (Monographies $/ \mathrm{ns}$ trumentum 24).

FRADKIN A. and LERNAU O.

in press The fishing economy at Caesarea Maritima, Israel. Joumal of Roman Archaeology, Stipplementary Series (Cuesarea Papers 3).

FREEDY K.S. and REDFORD D.B.

1970 The dates in Ezekiel in relation to Biblical, Babylonian and Egyptian sources. Joumal of the American Oriental Society' $90: 462-485$

FREESTONE 1 .

1994 Chemical analyses of raw fragments. In : HURST H.R.I. (ed.) Excasations at Carthage : the British Mission II. The Circular Harbour, North Side : 290. Oxford : Oxford University Press.

FrEESTONE I., GREENWOOD R. and GORIN-ROSEN Y.

2002 Byzantine and early Islamic glassmaking in the eastern Mediterranean : Production and distribution of primary glass. In: KORDAS G. (ed.), Hyalos-Vitrum-Glass. History, Technology and Conservation of Glass and Vitreous Materials in the Hellenic World: 167-174. Athens : Glasnet Publications.

FULFORD M.

1987 Economic interdependence among urban communities of the Roman Mediterranean. World Archaeology 19 : 58-75.

GAUTIER A.

1980 Contributions to the archacozoology of Egypt. In: WEN. DORF F. and SCHLD R. (eds), Prehistory of the Eastern Sahara: $317-344$. New York : Academic Press. 
The Early to Late Neolithic archeofaunas from Nabta and Bir Kiseiba. $I n$ : WENDORF F. and SCHILD R. (eds), Holocene Settlements of the Egyptian Sahara: The Archaeology of Nabta Playa: 609-635. New York and London: Plenum Press.

GAUTIER A. and VAN NEER IV.

1989 The animal remains from the Late Paleolithic sequence in Wadi Kubbaniya. $l n$ : WENDORF F., SCHILD R. and CLOSE A.E. (eds), The prehistory of Wadi Kubbaniya. Vol. 2, Stratigraphy, paleoeconomy and environment: 119-161. Dallas: Southern Methodist University Press.

GLUECK N.

1939 The second campaign at Tell el-Kheleifeh (Ezion-Geber: Elath). Bulletin of the American Schools of Oriental Research 75 (October) : 8-22.

GOPHNA R.

2002

Elusive anchorage points along the Israel littoral and the Egyptian Canaanite maritime route during the Early Bronze Age $1 . I n$ : LEVY T.E. and VAN DEN BRINK E.C.M. (eds), Egypt and the Levant. Interrelations from the $4^{\text {th }}$ through the early $3^{\text {rd }}$ Millennitum BCE: 418-421. London: Leicester University Press.

GOLDSMDT N.F., TCHERNOV E.G., GINSBURG L., TASSY P.

and VAN COUVERING J.A.

1982 Ctenodactylid rodents in the Miocene Negev fauna of Israel. Nattre 296: 645-647.

GREAVES A.M

2002 Miletos. A History. London : Routledge.

GREENWOOD P.H. and HOWES G.

1973 Fish remains. In: KARAGEORGHIS V. (ed.), Salamis $V$. Excavations in the necropolis of Salamis III: 259-268. Nicosia : Zevaltis Press.

\section{HAMLLTON-DYER S.}

2001 The faunal remains. $I n$ : PEACOCK D.P.S. and MAXFIELD V.A. (eds), Mons Claudiamus, Suvey and Excavation Vol. 2. Excarations Part 1:251-301. Cairo: IFAO (Doctuments de Fouilles 43).

1994 Preliminary report on the fish remains from Mons Claudianus, Egypt. Offa $51: 275-278$.

in press Faunal remains. $I n$ : PEACOCK D.P.S. and MAXFIELD V.A. (eds), Mons Porphyrites, Survey and Excavation: Vol 2 Finds. Exeter.

HANKEY $\mathrm{V}$.

1995

A Late Bronze Age temple at Amman Airport : small finds and pottery discovered in 1955. In: BOURKE $\mathrm{S}$. and DESCCEUDRES J.-P. (eds), Trade, Contact, and the Movememt of Peoples in the Eastern Mediterramean. Sydney: Meditarch (Mediterranean Archaeology' Stupplement 3).

HASEL M.G.

1998 Domination and Resistance: Egyptian Military Activity in the Southern Levant, ca. 1300-1185 BC. Leiden : Brill

HELCK W.

1961-1970 Materialien zur Wirtschaftsgeschichte des Netten Reiches. Wiesbaden: Akademie der Wissenschaften und der Literatur.

HERRMANN C.

1994 Ägyptische Amulette aus Palistina/Israel (mit einem Ausblick auf ihre Rezeption durch das Alte Testament).
Freiburg : University of Freiburg (Orbis Biblicts et Orientalis 138).

HODDER I. and ORTON C.

1976 Spatial Analysis in Archaeology. Cambridge : Cambridge University Press.

HOFFMANN R.C.

2001 Frontier foods for late medieval consumers : culture, economy, ecology. Environment and History $7: 131-167$

HORDEN P. and PURCELL N.

2000 The Corrupting Sea. A Study of Mediterranean History. Oxford : Blackwell Publishers.

HORWITZ L.K.

1987 Animal offerings from two Middle Bronze Age tombs. Isruel Exploration Joumal $37: 251-255$.

ILAN D.

1995 The dawn of internationalism - the Middle Bronze Age. $/ n$ : LEVY T.E. (ed.), The Archaeology of Society' in the Holy Lamd : 297-319. London : Leicester University Press.

JANSSEN J.J.

1961 Two Ancient Egyptian Ship's Logs. Leiden: Brill (Oudheidkundige Mededelingen uit het Rijksmuseum van Oudheden te Leiden Stuplement 42).

1975 Commodity Prices from the Ramessid Period. Leiden : Brilt.

KARAGIORGOU O.

2001 LR2 : a container for the military annona on the Danubian border. $l n:$ KINGSLEY S. and DECKER M. (eds), Economy and Exchange in the East Mediterranean during Late Antiquity': 129-166. Oxford : Oxbow Books.

KINGSLEY S.

2001 The economic impact of the palestinian wine trade in late antiquity. I $h$ : KINGSLEY S. and DECKER M. (eds), Economy and Exchange in the East Mediterranean during Late Antiquity : 44-68. Oxford : Oxbow Books.

KITCHEN K.A.

1986 The Third Intermediate Period (1 100.650 B.C.) Warminster : Aris and Phillips Ltd.

LECLANT J.

1958-1969 Fouilles et travaux en Égypte et au Soudan. Orientalia, Nova Series : 27-38.

LECLANT J, and CLERC G.

1985-1991 Fouilles et travaux en Egypte et au Soudan. Orientalia, Nova Series : $54-60$

LEGULLLOUX M

1997 Quelques aspects de l'approvisionnement en viande des praesidlia du Désert oriental égyptien. Archaeozoologia $9: 73-82$.

LEPIKSAAR J.

1995 Fish remains from Tell Hesban, Jordan. $m:$ LABIANCA $\emptyset . S$. and VON DEN DRIESCH A. (eds), Famal Remains: Taphonomical and Zooarchaeological Studies of the Animal Remains from Tell Hesban and 1/icinity: 171-210. Michigan : Andrews University Press.

LERNAU H.

1975 Animal remains, In: AHARONl Y. (ed.), Investigations at Lachish. The Sancturary and the Residency (Lachish V) : 86 103. Tel Aviv : Gateway Publishers Inc. 
1986 Fishbones excavated in two Late Roman - Byzantine castella in the southem desert of Israel. In: BRINKHUIZEN D.C. and CLASON A.T. (eds), Fish and Archaeology : 85-102. Oxford. (BAR Int. Ser: 294).

$1986 / 1987$ Subfossil remains of Nile perch (Lates cf. niloticus) ; first evidence from ancient Israel. Israel Jound of Zoology 34 : 225-236.

1988 Fish remains. $m:$ ROTHENBERG B. (ed.), The Egyptian Mining Temple at Timna: 241-246. London: Institute of Archieology, University College London.

in press Fish remains from Horvat Karkur.

LERNAU H. and LERNAU O.

1989 Fish bone remains. In: MAZAR E. and MAZAR B. (eds), Excarations in the South of the Temple Motm : the Ophet of Biblical Jenusalem: 155-161. Jerusalem: Institute of Archaeology, Hebrew University.

1992 Fisl remains. In : DE GROOT A. and ARJEL D.T. (eds), Excavations at the City of David 1978-1985: Final Report: 131148. Jerusalem : Institute of Archaeology, Hebrew University.

1994 The fish remains. In : LECHEVALLJER M. et RONEN A. (éd.), Le Site de Hatoula en Judée Occidenfale, /srael: $111-124$. Paris : Association Paléorient (Mémoires ef Trataux du Centre de Recherche Françuis de Jerusalem 8).

LERNAU O.

1995 The fish remains of Upper Zohar. $I n$ : HARPER R.P. (ed.), Upper. Zohar, an early byzantine fort in Palaestina Tertia. Final report of excavations in 1985-1986:99-111. Oxford: Oxford University Press.

1996 Fish remains from Tel Harassim. In: GivoN S. (ed.), The Sixth season of Excavation at Tel Harassim (Nahal Barkai) 1995: 14-23. Tel Aviv: Bar-Ilan University.

2000 Fish bones. In : FINKELSTEIN 1., USSISHKIN D. and HALPERN B. (eds), Megiddo 11 - the 1992-1996 Seasons: $463-$ 477. Tel Aviv: Emery and Claire Yass Publications in Archaeology, Institute of Archaeology.

2004 Fish Remains. In : FIGUERAS P. (ed.), Horvat Karkw' 'Illit : 335-349. Beersheva: Ben Gurion University of the Negev (Beersheva XV).

in press Remains of fish in archacological excavations in Ein Gedi.

LERNAU O., COTTON H. and GOREN Y. 1996 Salted fish and fish sauces from Masada. Archaeofatma 5 :
$35-41$.

LEVY T.E. and VAN DEN BRINK E.C.M.

2002 Interaction Models, Egypt and the Levantine Periphery. $I n$ : LEVY T.E. and VAN DEN BRINK E.C.M. (eds), Egypt and the Levant. Interrelations from the $4^{\text {th }}$ through the early $3^{\text {rd }} \mathrm{Mil}$ lemnitm BCE : 3-38. London: Leicester University Press.

LUND J.

1999 Trade patterns in the Levant from ca. $100 \mathrm{BC}$ to $\mathrm{AD} 200$ as reflected by the distribution of ceramic fine wares in Cyprus. Mïnstersche Beiträge za Antiken Handelsgeschichte IS : 1 22 .

MANHART H. and VON DEN DRIESCH A.

in press Tierreste der Bronze- und Eisenzeit von Kinneret (Tell elOreme), Israel. Doctmenta Archaeobiologiae 2.

MARCUS E.

2002

Early seafaring and maritime activity in the Soulhern Levant from prehistory through the third millennium BCE. $m$ : LEVY T.E. and VAN DEN BRINK E.C.M. (eds), Egypt and the Levant.
Interyelations from the $4^{\text {th }}$ through the early, $3^{\text {rd }}$ Willenniam $B C E$ : 401-417. London : Leicester University Press.

\section{MCGOVERN P.E.}

2000 The Foreign Relations of the "Hyksos" : A Nentron Activation Study of Midklle Bronze Age Pottery from the Eastern Mediternamean. Oxford : ArchaeoPress (BAR Int. Ser. 888).

MeigGs R.

1982 Trees and Timber in the Ancient Mediteranean World. Oxford: Clarendon Press.

MEYER-SCHLICHTMANN C.

1988 Die Pergemenisch Sigillafa aus der Stadtgrabung von Pergamon. Berlin : de Gruyter (Pergamenische Forschungen 6).

MILLARD A.R.

1970 Fragments of historical texts from Nineveh : Middle Assyrian and later kings. lroy $32: 167-176$, pl. 33 .

Mills A.J.

2002 Another Old Kingdom site in the Dakhleh oasis. In: FRICD. MANN R. (ed.), Egypt and Nubia. Gifts of the Desert : 65-69. London : British Museum Press.

MIROSCHEDJI P. DE

2002 The socio-political dynamics of Egyptian-Canaanite interaction in the Early' Bronze Age. $I n$ : LEVY T.E. and VAN DEN BRINK E.C.M. (eds), Egypt and the Levant. Interrelations from the $4^{\text {th }}$ through the early 3rd Milleminm BCE : 39-57. London: Leicester University Press.

MITCHELL $S$.

1993 Anatolia. Land, Men, and Gods in Asia Mtinor 1. The Celts and the Impact of Roman Rule : 136-142. Oxford : Oxford University Press.

MOENS L., DE PAEPE P. and WAELKENS M.

1997 An archaeometric study of the provenance of white marble sculptures from an Augustan Heroon and a middle Antonine Nymphaeum at Sagalassos. $m$ : WAELKENS $M$. and POBLoMe J. (eds), Sagalassos I'. Report on the Strvey and Excatation Campaigns of 1994 and 1995: 367-383. Leuven : Leuven University Press (Acta Archaeologica Lovamiensia Monographiae 9).

MUDAR K.

1982 Early Dymastic III animal utilisation in Lagash : a report on the fauna of Tell al-Hiba. Journal of Near Eastern Studies $41: 23-34$.

MUMFORD G.D.

1998 International Relations between Egypt, Sinai, and Sivia-Palestine during the Late Bronze Age to Early Persiul Period (D)masties 18-26: ca. 1550-525 BC). Toronto : University of Toronto, Ph.D. ; Ann Arbor : University Microfilms International.

MUNFFRD G. and PARCAK S.

2003 Pharaonic ventures into South Sinai : El-Markha Plain Site 346. The Jownal of Egyptian Archaeology $89: 83-116$.

NOLLÉ J.

1993 Side im Alternm. Geschichte und Zeugnisse I. Bonn : Habelt (Inschriflen griechischer Städte aus Kleinasien 43).

NÜMANN W.

1961 Die anatolischen Seen und ihre fishereiliche Bewirtschaftung. Zeitschrift fir Fischerei tut deren Hilfswissenschaften $10(8-10): 773-799$. 
ODED B.

1979 Mass Deportations and Deportees in the Neo-Assyrian Empire. Wiesbaden : Dr. Ludwig Reichert Verlag.

OPPENHEIM A.L.

1967 Essay on overland trade in the first miltennium BC. Journal of Cuneiform Studies 21 : 236-254.

OREN E.D.

1997a The "Kingdom of Sharuhen" and the Hyksos Kingdom. $m$ : OREN E.D. (ed.), The Hyksos: New Historical and Archoeological Perspectives : 253-284. Philadelphia : University of Pennsylvania Museum.

1997b Sinai. I $n$ : MEYers E.M. (ed.), The Oxford Encyclopedia of Archaeology in the Near East. Vol. 5: 41-46. New York: Oxford University Press.

PASZTHORY E.

1988 Laboratorien in Ptolemäischen Tempelanlagen. Eine naturwissenschaftliche Analyse. Antike Welt 19:3-20.

1990 Salban, Schminken und Parfime im Altertum. Herstellungsmethoden und Anwendungsbereiche im östlischen Mediterraneum. Antike Welt $21: 4-64$.

PEDEN A.J.

1994 Egyptian Historical Doctments of the Twentieth Dinasty. Jonsered : Paul Astroms forlag (Doctmenta Mundi Aegyptiaca 3).

PEstMan P.IV. (ed.)

1981 A Guide to the Zenon Archive. Leiden : Brill (Papyrologica Lugdumo-Batava 21).

PETERS J., PÖLLATH N and VON DEN DRIESCH A.

2002 Early and Late Bronze Age transitional subsistence at Tall al'Umayri. $h$ : HERR L.G., CLARK D.R., GERATY L.T., YOUNKER R.IV. and LABIANCA O.S. (eds), Madaba Plains Project 5: The 1994 Season at Tall al-'Umayt and Subsequem Studies: $305-347$. Michigan: Andrews University Press.

POBLONE $]$.

1999 Sagalassos Red Slip Ware. Typology and Chronology. Tumhout: Brepols (Studies in Eastern Mediterramean Archaeology 2).

POBLOME J. and WAELKENS M.

2003 Sagalassos and Alexandria. Exchange in the eastern Mediterranean. $I n$ : ABADIE-REYNAL C. (éd.), Les Céramiques en Anatolie aux Époques Hellénistique et Romaine: 179-191. Paris - Istanbul: Institut français d'études anatoliennes Georges Dumézil (Varia Anatolica 15).

PORTER B. and MOSS R.L.B.

1952 Topographical Bibliography of Ancien Egyptian Hieroglyphic Texts, Reliefs, and Paintings. Vol. VII. Nubia, the Deserts, and Outside Egypt. Oxford: Griffith Institute, Ashmolean Museum.

PRATICO G.D.

1985 Nelson Glueck's 1938-1940 excavations at Tell elKheleifeh : a reappraisal. Bulletin of the American Schools of Oriental Research 259 (summer/August) : 1-32.

PRITCHARD J.B. (ed.)

1969 Ancient Near Eastern Texts Relating to the Otd Testament. Princeton: The University Museum, University of Pennsylvania.
QUAEgebeur J. et TRAUNECKer C. en collaboration avec CASSEYAS C., Chartier-RAymond M., CreEmers G., Dils P. et RoOvers I.

1994 Chenhour 1839-1993. État de la question et rapport des travaux de 1992 et de 1993. Cahier's de Recherches de l'Instittut de Papyrologie et d'Egyptologie de Lille 16: 167-209.

\section{REDFORD D.B.}

1992 Egypt, Canaan, and Israel in Ancient Times. Princeton : Princeton University Press.

REESE D.

1991 The trade of Indo-Pacific shells into the Mediterranean basin and Europe. Oxford Jownal of Archaeology $10: 159-196$.

REESE D., MIENIS H.K. and WOODWARD F.

1986 On the trade of shells and fish from the Nile River. Bulletin of the American Schools of Oriental Reseurch $264: 79-84$.

\section{RODRIGO GARCIA M.J.}

1994 Remains of Melumogrammus aeglefimus (Linnaets, 1758) in the Pleistocene-Holocene passnge of the Cave of Nerja, Malaga/Spain. Offa $51: 348-351$.

ROSE M.J.

1994 With Line and Glittering Bronze Hook: Fishing in the Aegean Bronze Age. Bloomington : Indiana University, Doctoral thesis.

ROSELLÓ IZQUIERdo E. and MORALES MUÑ A.

2001 Fish offerings from Tell Brak ? Comments on an articulated specimen found in the 1990 field season. $m$ : OATES D., OATES J. and MCDONALD H. (eds), Excarutions at Tell Brak. Vol. 2: Nagur in the Third Millemium BC:339.345. Ankara : The British Institute of Archaeology / Cambridge : McDonald Institute for Archacological Research.

ROTHENBERG B.

1972 Were These King Solomon's Mines? Excavations in the Timna Valley. New York: Stein and Day Publishers.

1993 Timna'. I $m$ : STERN E., LEWINSON-GLLBOA A. and AVIRAM J. (eds), The New Encyclopedia of Archaeological Excavafions in the Holy Land $4: 1475-1486$. Jerusalem : The Israel Exploration Society.

RzósKA J.

1980 Euphrates and Tigris, Mesopotamian Ecologv and Destiny. The Haguc : Dr. W. Junk Publishers.

SAFRAI Z

1994 The Economy of Roman Palestine. London: Routledge.

SCHEERS S.

2000 Coins found during 1996 and 1997. In : WAELKENS M. and Loots L. (eds), Sagalassos V. Report on the Survey and Excavation Campaigns of 1996 and 1997: 509-549. Leuven: Leuven University Press (Acta Archaeologica Losaniensia Monographiae (1).

SHARVIT J., GALILI E., ROSEN B. and VAN DEN BRINK E.C.M.

2002 Predynastic maritime traftic along the Carmel coast of Israel : a submerged find from North Atlit Bay. $l n:$ VAN DEN BRINK E.C.M. and YANNAI E. (eds), In Quest of Ancient Settlements and Landscapes: 159-166. Jerusalem: Israel Exploration Society.

SIDEBOTHAM S. and ZITTERKOPF R.E.

1995 Routes through the Eastem Desert of Egypt. Expedition 37 : $39-52$. 
SNEH A, and WEISSBROD T.

1973 Nile Delta : the defunct Pelusiac branch identified. Science $180: 59-61$.

SODINI J.-P.

2000

Productions et échanges dans le monde protobyzantin : le cas de la céramique. $I n$ : BELKE K. (Hrsg.), Byzans als Ramm. Zu Methoden und Inhalten der historischen Geographie des östlichen Mittelmeerraumes: 181-208. Vienna : Österreichische Akademie der Wissensehaften, Philosophisch-Historische Klasse.

SPERBER D.

1976 Objects of trade between Palestine and Egypt in Roman times. Joumal of the Economic and Social History of the Orient $19: 113-147$.

1998 The City in Roman Palestine. Oxford: Oxford University Press.

STAHL U.

1989 Tierknochenfinde vom Hassek Höyïk. Institut fir Palaeoanatomie, Domestikationsforschung und Geschichte der Tiermedizin der Universität München, Doctoral thesis,

\section{STERN E.}

2001 Archaeology' of the Land of the Bible, volume 1I. The Assyrian, Bubylonian, and Persian Periods 732-332 BCE. New York : Doubleday.

\section{STUDER J.}

1994

Roman fish sauce in Petra, Jordan. $l n$ : VAN NEER W. (ed.), Fish Exploitation in the Past. Proceedings of the $7^{\text {th }}$ Meeting of the ICAZ Fish Remains Working Grotp: 191-196. Tervuren : Musée Royal de l'Afrique Centrale (Annales du Musée Royal de l'Afrique Centrale, Sciences Zoologiques 274).

TALLOEN P.

2001 The Egyptian connection. The cult of Nilotic deities at Sagalassos. Ancient Society $31: 289-327$.

TEMIN P.

2001

A market economy in the early Roman empire. The Jownat of Roman Studies $91: 169-181$.

THORSON T.B.

1974 Occurrence of the sawfish, Pristis peroteti, in the Amazon River, with notes on P. pectinatus. Copeia 1974:560-564.

TOMBER R.S.

1996 Provisioning the desert : pottery supply to Mons Claudianus. In: BAILEY D.M. (ed.), Archaeological Research in Roman Egypt. Joumal of Roman Archaeology. Supplement 19:3949. Ann Arbor, MI.

\section{VANDEPUT L.}

1997 The Architectural Decoration in Roman Asia Minor: Sagalassos: a Case Siudy. Turnhout: Brepols (Sudies in Eastem Mediterranean Archaeology 1).

VAN ELSBERGEN M.J.

1997 Fischerei im Alten Ägypten. Untersuchumgen zu den Fischfangtarstellungen in den Gräbern der 4. bis 6. Dynastie. Berlin : Achet-Verlag.

VAN NEER $W$.

1984 Les restes de poissons de quelques maisons d'Apamée. $I n$ : BALTY J. (éd.), Apamée de Syrie. Bilan des Recherches
Archéologiques 1973-1979. Aspects de l'Architecture Domestique d'Apamée: 291-303. Bruxelles. (Foulles d'Apamée de Syrie. Miscellanea 13).

1989 Recent and fossil fish from the Sahara and their palaeohydrological meaning. Palueoecology of Africa $20: 1-18$.

1993 Fish remains from the Last Interglacial at Bir Tarfawi (Eastern Sahara, Egypt). $I n$ : WeNdorf F., SCHILd R., CLOSE A.E. and associates (eds), Egypt during the Last Interglacial. The Middle Paleolithic of Bir Tarfawi and Bir Sahara East : 144 154. New York \& London: Plenum Press.

VAN NEer W., DE CUPERE B. and WAELKENS $M$.

1997 Remains of local and imported fish at the ancient site of Sagalassos (Burdur Prov., Turkey). In: WAELKENS M. and POBLOME J. (eds), Sagalassos W. Report on the Survey and Excavation Campaigns of 1994 and 1995: 571-586. Leuven: Leuven University Press (Acta Archaeologica Lovaniensia Monographiae 9).

VAN NEER W., DUFOUR E. and WAELKENS M.

2004 Stable isotope analysis as a means of establishing the origin of imported fish. Paper presented at the $7^{\text {th }}$ International Meeting of the ASWA Working Group (Archaeozoology of Southwest Asia and Adjacent Areas), Ankara, 31 May - 4 June 2004.

VAN NEER W. and ERVYNCK A.

1998 The faunal remains. $I n$ : SIDEBOTHAM S. and WENDRICH W. (eds), Berenike '96. Report of the Excavations at Berenike (Egytian Red Sea Coast) and the Strvey' of the Eastem Desert : 349-388. Leiden (CNWS Publications. Special Series 3).

1999 The faunal remains. In : SIDEBOTHAM S. and WENDRICH W. (eds), Berenike '97. Report of the 1997 Excavations at Berenike and the Survey of the Egyptian Eastern Desert, inchiding Excavations at Shenshef: 325-348. Leiden (CNW'S Publications. Special Series 4).

2004 Remains of traded fish in archaeological sites : indicators of status or bulk food? In: O'DAY S.J., VAN NEER IV. and ERVYNCK A. (eds), Behaviour Behind Bones. The Zoonchaeology of Ritual, Religion, Status and Identity : 203-214. Oxford : Oxbow Books.

VAN NEER W., PAULISSEN E. and VERMEERSCH P.M.

2000a Chronology, subsistence and environment of the Late Palaeolithic fishing sites of Makhadma-2 and 4. $I n:$ VERMEERSCH P.M. (ed.), Palaeolithic Living Sites in Upper and Middle Egypt : 271-287. Leuven : Leuven University Press.

VAN NEER W., WILDEKAntP R.H., KOÇŨK F., ÜNLŪSAYIN M., WAELKENS M. and PAULISSEN E.

$2000 \mathrm{~b} \quad$ Results of the 1996 survey of the fish fauna of the Aksu river and some lakes in southwestern Anatolia, and the implications for trade at Sagalassos. $m$ : WAELKENS M. and LOOTS L. (eds), Sagalassos V. Report on the Survey and Excavation Campaigns of 1996 and 1997 : 828-842. Leuven : Letwen University Press (Acta Archaeologica Lovaniensia Monographiae 11).

VAN NEER W., WILDEKaMP R., WAELKENS M., ARNDT A. and VOLCKAERT $F$.

$2000 \mathrm{c} \quad$ Fish as indicators of trade relationships in Roman times: the example of Sagalassos, Turkey. $I n$ : MASHKOUR M., CHOYKE A.M., BUITENHUIS H. and POPLIN F. (eds), Archaeozoology of the Near East IV. Proceedings of the Fourth International Sympositm on the Archaeozoology of Southwestern Asia and Adjacent Areas : 206-215. Groningen (ARC-Publication 32). 
VAN NEER W. and DE CUPERE $B$.

2001 Faunal remains from Tell Beydar (excavation seasons 19921996 and 1997 partim). In : VAN LERBERGHE K. and VOET G. (eds), Tell Beydar Environmental and Technical Studies : 69-115. Turnhout : Brepols (Subartu 6).

VAN NEER W. and WAELKENS $M$.

in press Fish remains from Bronze Age to Byzantine levels at Kilise Tepe (Turkey). $m$ : POSTGATE J.N. and THOMAS D.C. (eds), Excavations al Kilise Tepe, 1994-1998. Ankara : The British Institute of Archacology / Cambridge : McDonald Institute for Archacological Research.

VAN NEER W., WILDEKAMP R.H., KÜÇŌK F., ÜNLÖSAY'iN M., POBLOME J. and WAELKENS M.

in press The 1997-1999 surveys of the Anatolian fish fatna and their relevance to the interpretation of trade at Sagalassos. In: WAELKENS M. and POBLOME J. (eds), Sagalassos $1 / \%$. Report on the Survey and Excavation Campaigns of 1998, 1909 and 2000. Leuven: Leuven University Press (Acta Archaeologica Lov'aniensia Monographita).

VIGNE J.-D. et BUITENIUIS H.

1999 Les premiers pas de la domestication animale à l'Ouest de l'Euphrate : Chypre et l'Anatolie centrale. Paléorient 25,2: 49-62.

VINCIGUERRA D.

1922 Missione zoologica del Dott. E. Festa in Cirenaica. Pesci. Bolletino dei Musei di Zoologia ed Anatomia comparata della $R$. Universita di Torino $37: 1-6$.

VON DEN DRIESCH A.

1981 Fischknoclsen. In: HROUDA B. (ed.), Isim-Isan Bahriyat II : 157-167. München : Verlag der Bayerischen Akademie der Wissenschaften (Bayerische Akatemie der Wissenschaften, Philosophisch-Historische Klasse, Abhandlingen - Nene Folge 87).

1983

Some archaeozoological remarks on fishes in Ancient Egypt. In: GRIGSON C. and CLUTTON-BROCK J. (eds), Animals and Archaeology. 2. Shell Middens, Fishes and Birds: 87-110. Oxford. (BAR Imt. Ser. 183).

1986a Fischknochen aus Abu Salabikh, Iraq. lraq $68: 31-38$.

1986b Fische im alten Ägypten - eine osteoarchäologische Untersuchung. Doctmenta naturae $34: 1-33$.

VON DEN DRIESCH A. and BOESSNECK J.

1995 Final report on the zooarchaeological investigation of animal bone finds from Tell Hesban, Jordan. $n$ : LABIANCA $\emptyset . S$. and VON DEN DRIESCH A. (eds), Famal Remains : Taphonomical and Zooarchaeological Studies of the Animal Remains from Tell Hesban and Vicinity : 65-108. Michigan : Andrews University Press.

WAELKENS $M$

1982 Dokimeion. Die Werkstatt der reprïsentativen kleinasiatischen Sarkophage. Berlin : Mann Verlag. (Archïologische Forschingen 11).
2002 Romanization in the East. A case study : Sagalassos and Pisidia. Istanbuler Mitteilungen $52: 311-368$.

in press Ein Blick von der Ferne. Seleukiden und Attaliden in Pisidien. Istanbuler Mitteilungen 54.

WAELKENS $M$. and the SAGALASSOS TEAM

1997 Interdisciplinarity in classical archaeology. A case study : the Sagatassos Archaeological Research Project. $I n$ : WAELKENS M. and POBLOME J. (eds), Sagalassos $\mathrm{IV}$. Report on the Survey and Excavation Campaigns of 1994 and 1995: 225-252. Leuven: Leuven University Press (Actu Archaeologica Lovaniensia Monographiae 9).

WAELKENS M., MUChez Ph., LOOTS L., DegrySE P., VANDEPUT L., ERCAN S., MOENS L. and DE PAEPE P.

2002 Marble and the marble trade at Sagalassos (Turkey). $I n$ : HERRMANN J.J., HERZ N. and NEWMAN R. (eds), mlerdisciplinary studies on ancient stone: $370-380$. London: Archetype Publications Ltd.

WENDORF F., SCHILD R., CLOSE A.E. and ASSOCIATES

1993 Egypt during the Last Interglacial. The Middle Palcolithic of Bir Tarfawi and Bir Sahara East. New York \& London: Plenum Press.

IVEINSTEIN J.M.

1975 Egyptian relations with Palestine in the Middle Kingdom. Bulletin for the American Schools of Oriental Research 217 1-16.

1981 The Egyptian Empire in Palestine : a reassessment. Bulletin for the American Schools of Oriental Research 24 I : 1-16.

WHEELER A.

1979 The fish bones, In: LLOYD J.A. (ed.), Excavations at Sidi Khrebish, Benghazi (Berenice) 2 : 25-26. Tripoli : Department of Antiquities (Stpplement to Liby'a Antiqua 5.2).

WOOLLEY C.L.

1921 Carchemish part II: The Town Defences. London : British Museum Publications.

YEKUTIELL Y.

2002 Settlement and subsistence patterns in North Sinai during the fifth to third millennia BCE. $I n:$ LEVY T.E. and VAN DEN BRINK E.C.M. (eds), Egypt and the Levant. Interrelations from the $4^{\text {th }}$ through the early $3^{\text {rd }}$ Millennitu $B C E: 422-436$. London : Leicester University Press.

YOUNG G.K.

$2001 \quad$ Rome's Eastern Trate. International Conmerce and Imperial Policy. London : Routledge.

ZIEGLER R. Und BOESSNECK 3 .

1990 Tierreste der Eisenzeit II. In : FRITZ V. (Hrsg.), Kinneret. Ergebnisse der Ausgrabungen auf dem Tell-el'Oreme am See Gennesaret 1982-1985: 133-158. Wiesbaden : Harrassowitz. 